\title{
Advanced Aeroelastic Simulations for Practical Fixed-wing and Rotary-wing Applications
}

by

\author{
Sean A. M. McTavish \\ A Thesis submitted to \\ the Faculty of Graduate Studies and Research \\ in partial fulfilment of \\ the requirements for the degree of \\ Master of Applied Science \\ Ottawa-Carleton Institute for \\ Mechanical and Aerospace Engineering \\ Department of Mechanical and Aerospace Engineering \\ Carleton University \\ Ottawa, Ontario, Canada \\ September 2008 \\ Copyright (c) \\ 2008 - Sean A. M. McTavish
}




$\begin{array}{ll}\begin{array}{l}\text { Library and } \\ \text { Archives Canada }\end{array} & \begin{array}{l}\text { Bibliothèque et } \\ \text { Archives Canada }\end{array} \\ \begin{array}{l}\text { Published Heritage } \\ \text { Branch }\end{array} & \begin{array}{l}\text { Direction du } \\ \text { Patrimoine de l'édition }\end{array} \\ \begin{array}{l}\text { 395 Wellington Street } \\ \text { Ottawa ON K1A 0N4 } \\ \text { Canada }\end{array} & \begin{array}{l}\text { 395, rue Wellington } \\ \text { Ottawa ON K1A 0N4 } \\ \text { Canada }\end{array}\end{array}$

Your file Votre référence ISBN: 978-0-494-44052-0 Our file Notre référence ISBN: 978-0-494-44052-0

NOTICE:

The author has granted a nonexclusive license allowing Library and Archives Canada to reproduce, publish, archive, preserve, conserve, communicate to the public by telecommunication or on the Internet, loan, distribute and sell theses worldwide, for commercial or noncommercial purposes, in microform, paper, electronic and/or any other formats.

The author retains copyright ownership and moral rights in this thesis. Neither the thesis nor substantial extracts from it may be printed or otherwise reproduced without the author's permission.
AVIS:

L'auteur a accordé une licence non exclusive permettant à la Bibliothèque et Archives Canada de reproduire, publier, archiver, sauvegarder, conserver, transmettre au public par télécommunication ou par l'Internet, prêter, distribuer et vendre des thèses partout dans le monde, à des fins commerciales ou autres, sur support microforme, papier, électronique et/ou autres formats.

L'auteur conserve la propriété du droit d'auteur et des droits moraux qui protège cette thèse. $\mathrm{Ni}$ la thèse ni des extraits substantiels de celle-ci ne doivent être imprimés ou autrement reproduits sans son autorisation.
In compliance with the Canadian Privacy Act some supporting forms may have been removed from this thesis.

While these forms may be included in the document page count, their removal does not represent any loss of content from the thesis.
Conformément à la loi canadienne sur la protection de la vie privée, quelques formulaires secondaires ont été enlevés de cette thèse.

Bien que ces formulaires aient inclus dans la pagination, il n'y aura aucun contenu manquant.

\section{Canada}




\section{Abstract}

The study of aeroelasticity has many applications in the aerospace industry. There is a need in the fixed-wing and rotary-wing fields to develop computational aeroelastic tools for industrial applications that are both rapid and robust. Aeroelastic tools that would benefit the industry were developed in this work to predict the transonic fixed-wing flutter boundary and to predict rotary-wing wind turbine performance.

The flutter boundary of a wing must be determined during development and certification of an aircraft, and is critical in the transonic regime, where nonlinear effects create a dip in the flutter boundary that cannot be predicted with traditional linear tools. A frequency domain correction procedure was developed to account for nonlinear aerodynamics in the transonic regime. The flutter boundary of the experimental benchmark AGARD 445.6 wing was calculated using time domain and corrected frequency domain methods. Both approaches adequately predicted the flutter boundary, but the corrected frequency domain approach is significantly faster than the time domain simulations and represents a unique opportunity for improved flutter prediction during aircraft wing design and development.

Wind turbines represent a rapidly growing source of renewable energy but current predictive tools have been shown to lack accuracy in predicting the power output of wind turbines. Additionally, wind farm performance must be properly predicted to develop accurate annual energy estimates. An aeroelastic, aeroacoustic, discrete vortex method code called SMARTROTOR was used to predict the performance of 
the benchmark National Renewable Energy Laboratory (NREL) wind turbine experiment. The code properly predicted the NREL wind turbine performance in normal and yawed flow conditions and has demonstrated the capability of simulating the wake interference effects present in wind farms. The grid-free characterization of the wake behind the turbine and the rapid simulation time compared with grid-based computational fluid dynamics solvers highlights the relevance of the code for industrial applications. 
This thesis is dedicated to my grandmothers and the inspiration they have provided. 


\section{Acknowledgments}

I would like to thank Dr. Daniel Feszty and Dr. Fred Nitzsche for their support and encouragement throughout this project. Thank you to all of the professors in the Mechanical and Aerospace Engineering department at Carleton who have been

wonderful teachers and mentors and to Nancy Powell, Marlene Groves, and Christie Egbert, whose help has been invaluable throughout my undergraduate and graduate degrees. I would like to extend my appreciation to Dr. Slavik and my colleagues at the Czech Technical University in Prague who made my experience there even more enjoyable.

Thank you to my friends, roommates, colleagues, and officemates who have all made this a tremendous experience. Thank you to Peter Klimas, whose dedication and conversations helped make the many late nights working at Carleton so enjoyable.

I am grateful for the endless uplifting support shown by my parents, my brother, and my sisters. Lastly, I need to thank Josée Rivard, whose support, thoughtfulness and encouragement I appreciate so much. 


\section{Table of Contents}

$\begin{array}{ll}\text { Abstract } & \text { ii }\end{array}$

Acknowledgments $\quad$ v

Table of Contents vi

List of Tables $\quad \mathbf{x}$

List of Figures $\quad$ xi

Nomenclature $\quad$ xiv

1 Introduction 1

1.1 Motivation ...................... 1

1.1.1 Fixed-wing Aeroelasticity _. . . . . . . . . . . . 2

1.1.2 Rotary-wing Aeroelasticity . . . . . . . . . . . . 3

1.2 Objectives of the Thesis . . . . . . . . . . . . 4

1.3 Organization of the Thesis . . . . . . . . . . . 4

I Fixed-wing Aeroelasticity 6

2 Time Domain and Frequency Domain Flutter Solutions $\quad 7$

2.1 Description of Flutter . . . . . . . . . . . . . . 7 
2.2 Time Domain Analysis . . . . . . . . . . . . . . . . . . 12

2.3 Uncorrected Frequency Domain Analysis . . . . . . . . . . . . 17

2.3.1 Aerodynamic Modeling . . . . . . . . . . . . . . . 18

2.3 .2 Aeroelastic Modeling . . . . . . . . . . . . . . . . . . . 21

2.4 Corrected Frequency Domain Analysis _ . . . . . . . . . . 25

3 Flutter Simulation Results $\quad 29$

3.1 AGARD 445.6 Test Case . . . . . . . . . . . . . . . . . 29

3.1.1 Aerodynamic and Structural Grids . . . . . . . . . . . 30

3.1 .2 Test Conditions . . . . . . . . . . . . . . . . . . 34

3.2 Time Domain Results . . . . . . . . . . . . . . . . . . 35

3.3 Uncorrected Frequency Domain Results . . . . . . . . . . . . . 38

3.4 Corrected Frequency Domain Results . . . . . . . . . . . . . . 39

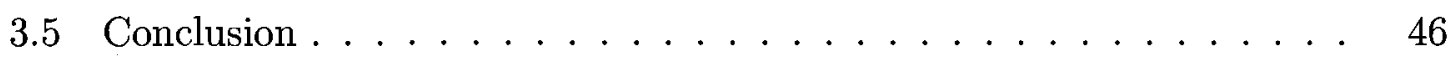

3.6 Recommendations and Future Work . . . . . . . . . . . . 46

II Rotary-wing Aeroelasticity $\quad 48$

4 Introduction to Wind Turbine Technology 49

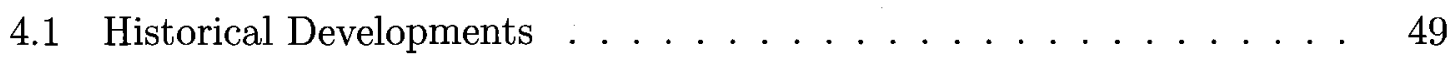

4.2 Anatomy of a Horizontal Axis Wind Turbine . . . . . . . . . 53

4.3 Power Generation . . . . . . . . . . . . . . . . . . . 54

4.4 Aerodynamic and Structural Loading . . . . . . . . . . . . . 58

4.5 Wind Farm Effects $\ldots \ldots \ldots \ldots \ldots$

5 A Review of Wind Turbine Aeroelasticity 63

5.1 Wind Turbine Wake Modeling . . . . . . . . . . . . . . 64

5.1 .1 Wake Models . . . . . . . . . . . . . . . . . 64 
5.1.2 Wind Farm and Wake Modeling Software . . . . . . . . 66

5.1 .3 Wake Model Validation . . . . . . . . . . . . . . 67

5.2 Aerodynamic Modeling Techniques . . . . . . . . . . . . . . 69

5.2.1 Blade Element Momentum Theory . . . . . . . . . . . . . 69

5.2 .2 Actuator Disk Techniques . . . . . . . . . . . 70

5.2 .3 Potential Flow Methods . . . . . . . . . . . . 70

5.2 .4 Computational Fluid Dynamics . . . . . . . . . . . 74

5.2 .5 Hybrid CFD/DVM Analysis . . . . . . . . . . . 76

5.3 Structural Modeling Techniques . . . . . . . . . . . 77

5.4 Motivation for the current work:

The NREL Experiment . . . . . . . . . . . . . . . . 78

6 Aerodynamic and Aeroelastic Theory in SMARTROTOR $\quad 81$

6.1 Aerodynamic Model . . . . . . . . . . . . . . . . . . 81

6.1 .1 GENUVP Theory . . . . . . . . . . . . . . . . 82

6.1.2 Panel Method - Solving for $\vec{u}_{\text {solid }}$ and $\vec{u}_{\text {near-wake }} \ldots \ldots . . .83$

6.1.3 Discrete Vortex Method - Solving for $\vec{u}_{f a r-w a k e} \ldots \ldots \ldots 8$

6.1.4 Coupling Between the Near- and Far-Wakes . . . . . . 89

6.2 The ONERA Dynamic Stall Model _ . . . . . . . . . . . . . 89

6.3 Rotor Blade Structural Modeling . . . . . . . . . . . . . . . 94

6.3.1 Structural Model Theory and Implementation . . . . . . . . 95

6.3.2 Aeroelastic Coupling with GENUVP . . . . . . . . . 9 96

$\begin{array}{llr}7 & \text { Results } & 99\end{array}$

7.1 Rigid Body Aerodynamic Results . . . . . . . . . . . . . . . 99

7.1.1 S809 Static Lift Curve . . . . . . . . . . . . . . . 100

7.1.2 S809 Unsteady Simulations _. . . . . . . . . . . . . . 102

7.2 Aeroelastic Results . . . . . . . . . . . . . . . . . 107 
7.2.1 NREL UAE Phase VI Test Case . . . . . . . . . . . . 107

7.2 .2 Effects of Yaw Misalignment . . . . . . . . . . . . 115

7.2 .3 Wake Interference Effects . . . . . . . . . . . . . . . . . 117

7.3 Conclusions . . . . . . . . . . . . . . . . . . . . . 119

7.4 Recommendations and Future Work . . . . . . . . . . . . . . . . 121

List of References $\quad 124$

Appendix A Derivation of the AIC-correction Fourier Transform 135

Appendix B Geometric and Structural Properties of the NREL UAE

$\begin{array}{ll}\text { Phase VI Wind Turbine } & 138\end{array}$

Appendix C Implementation of the ONERA dynamic stall model 142

Appendix D Changes made to the SMARTROTOR Source Code 148 


\section{List of Tables}

3.1 Comparison of modal frequencies for the AGARD 445.6 wing. . . . . 34

3.2 Flow conditions for the AGARD 445.6 flutter tests. . . . . . . . . 35

3.3 Flutter coefficient comparison at Mach 1.072. . . . . . . . . . 37

3.4 Flutter coefficient comparison at Mach 0.99. . . . . . . . . . . 42

4.1 AWEA Wind Classes at a height of $50 \mathrm{~m} . \ldots \ldots$

7.1 S809 static lift curve test conditions . . . . . . . . . . . . . . 101

7.2 S809 unsteady lift curve test conditions . . . . . . . . . . . . . 103

7.3 NREL turbine simulation parameters. . . . . . . . . . . . 108

7.4 Wake interference test conditions. . . . . . . . . . . . 117

B.1 Interpolated geometric parameters for the NREL turbine blade. . . 139

B.2 Interpolated structural parameters for the NREL turbine blade. . . . 141

C.1 ONERA dynamic stall coefficients. . . . . . . . . . . . . . 147 


\section{List of Figures}

1.1 Deflection of the wind turbines at the Horns Rev wind farm. . . . . . 4

2.1 Amplitude response of a wing. . . . . . . . . . . . . 8

2.2 Schematic of the flutter boundary of an aircraft. . . . . . . . . 10

2.3 Nonlinear effects over a wing in transonic flow. . . . . . . . . . . . 11

2.4 Output from time domain and frequency domain methods used to calculate a single flutter point. . . . . . . . . . . . . 13

3.1 The weakened AGARD 445.6 experimental model. . . . . . . . . . . 30

3.2 Grid topologies used in the Euler and RANS simulations. . . . . . . . 32

3.3 Time domain flutter boundary for the AGARD 445.6 wing. . . . . . . 36

3.4 Uncorrected frequency domain flutter boundary for the AGARD 445.6

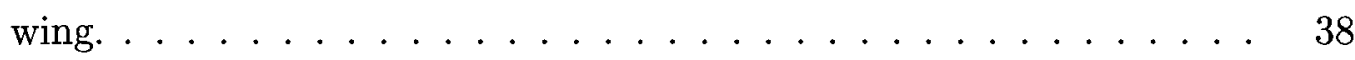

3.5 Real and imaginary unsteady pressures for the AGARD 445.6 wing . 41

3.6 Amplitude and phase of the unsteady pressures at Mach 0.960 . . . . 42

3.7 AIC-corrected velocity-frequency and velocity-damping plots at Mach 0.678 and Mach $0.901 . \ldots . \ldots . \ldots . \ldots . . \ldots 43$

3.8 AIC-corrected velocity-frequency and velocity-damping plots at Mach 0.660 and Mach 0.990 . . . . . . . . . . . . . . . . 44

3.9 Corrected flutter boundary for the AGARD 445.6 wing. . . . . . . . . 45

4.1 The development of wind turbines. . . . . . . . . . . . . 52

4.2 Growth of modern horizontal axis wind turbines. . . . . . . . . 53 


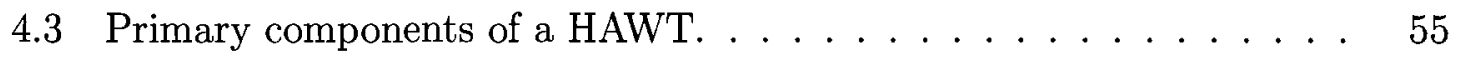

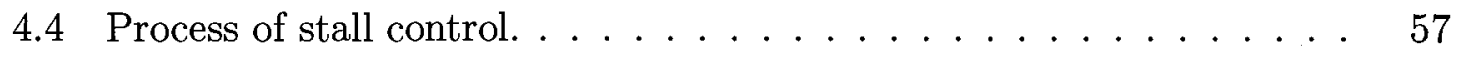

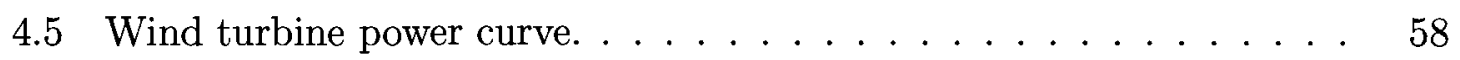

4.6 Wakes behind the NREL turbine in steady and unsteady environments. 59

4.7 Representation of the loads and stresses on a turbine rotor. . . . . . 60

4.8 Power curve of an isolated turbine and of a turbine in a wind farm. . 62

5.1 Schematic of the PARK wake model. . . . . . . . . . . 65

5.2 Schematic of the Ainslie wake model. . . . . . . . . . . . . . 65

5.3 Vortex wake representation with a vortex lattice model and a vortex particle $\operatorname{method} \ldots \ldots \ldots \ldots \ldots \ldots$. . . . . . . . . . 71

5.4 The NREL $10 \mathrm{~m}$ diameter wind turbine. . . . . . . . . . 79

5.5 Predicted and measured turbine torque from the NREL blind comparison. 80

6.1 The coupling between the near and far wakes. . . . . . . . . 90

6.2 Effect of dynamic stall on the lift coefficient. . . . . . . . . . . 91

7.1 Profile of the S809 Airfoil . . . . . . . . . . . . . 100

7.2 Static lift curve for the S809 airfoil. . . . . . . . . . . . . . . . . 101

7.3 Visualization of the wake with an $8^{\circ}$ angle of attack. . . . . . . 102

7.4 Original unsteady lift curve with a reduced frequency of $0.077 \ldots \ldots 105$

7.5 Unsteady lift curve: $8 \pm 5.5^{\circ} \ldots \ldots \ldots \ldots \ldots \ldots$

7.6 Unsteady lift curve: $14 \pm 5.5^{\circ} \ldots \ldots \ldots \ldots \ldots \ldots$

7.7 Chord and twist distribution for the NREL blade. . . . . . . . . 109

7.8 Convergence behaviour of the NREL blade torque. . . . . . . . 110

7.9 Visualization of the NREL turbine wake. . . . . . . . . . . 111

7.10 Comparison of the NREL turbine torque. . . . . . . . . . . 113

7.11 Comparison of the predicted NREL turbine power. . . . . . . . 114

7.12 Comparison of the predicted NREL turbine thrust. . . . . . . . . 114

7.13 Comparison of the predicted NREL turbine power in yawed flow. . . 116 
7.14 Comparison of the predicted NREL turbine thrust in yawed flow. . . 116

7.15 Top view of the NREL wake predicted by SMARTROTOR at three yaw angles. ............................. 118

7.16 Wake interaction in a closely spaced wind farm configuration. . . . . 120

C.1 Lift, Drag, and Moment curves used in the ONERA model. . . . . . . 144 


\section{Nomenclature}

This thesis uses SI units for consistency with the current wind energy industry practice. The nomenclature has been separated according to the two parts of the thesis.

\section{Part I - Fixed-wing Aeroelasticity}

$[A I C]$ Aerodynamic influence coefficients matrix

$\Delta \alpha \quad$ Amplitude of the angle of attack

$b_{s} \quad$ Root semi-chord

$\Delta C_{p} \quad$ Lifting pressure coefficient

$[D] \quad$ Downwash matrix

e Semi-width of a panel element

$E_{1} \quad$ Longitudinal elastic modulus

$E_{2} \quad$ Lateral elastic modulus

$[F] \quad$ Substantial derivative operator

$\varphi_{j}^{0} \quad$ Blending function

[Ф] Modal transformation matrix 


\begin{tabular}{|c|c|}
\hline$G$ & Shear modulus \\
\hline$h$ & Displacement mode shape vector \\
\hline$[H]$ & Aerodynamic transfer function matrix \\
\hline$k_{r}$ & Reduced frequency \\
\hline$[K]$ & Structural stiffness matrix \\
\hline$K$ & Kernel function \\
\hline$\left\{L_{a}\right\}$ & Aerodynamic force vector \\
\hline$\left\{L_{e}\right\}$ & External force vector \\
\hline$[M]$ & Structural mass matrix \\
\hline$N L$ & Nonlinear \\
\hline$\nu$ & Poisson's ratio \\
\hline$\{q\}$ & Generalized coordinate vector \\
\hline$q_{\infty}$ & Dynamic pressure \\
\hline$[Q]$ & Aerodynamic loads matrix \\
\hline$R e, I m$ & Real and imaginary components of a complex number \\
\hline$\rho$ & Density \\
\hline$s$ & Laplace variable, $s=i \omega$ \\
\hline$[S]$ & Integration matrix \\
\hline
\end{tabular}


$t \quad$ Time

$\tau \quad$ Non-dimensional time

$\{u\} \quad$ Structural displacement vector

$\bar{U} \quad$ Flutter speed coefficient

$U_{\infty} \quad$ Freestream velocity

$W \quad$ Normal velocity at surface

$[W T] \quad$ Weighting matrix

$\omega \quad$ Oscillation frequency

$x, y, z \quad$ Cartesian coordinates of a general field point

$\xi, \eta, \zeta \quad$ Coordinates of a point pressure doublet

AIC Aerodynamic Influence Coefficients

CFD Computational Fluid Dynamics

CMB Carleton Multi-Block

CVT Constant Volume Tetrahedron

DLM Doublet-Lattice Method

LCO Limit Cycle Oscillation

RANS Reynolds Averaged Navier-Stokes

TFI Transfinite Interpolation

TSD Transonic Small Disturbance 


\begin{tabular}{ll} 
Part II - Rotary-wing Aeroelasticity \\
$\alpha$ & Angle of attack, scalar potential \\
$\alpha_{c r i t}$ & Critical angle of attack \\
$\beta$ & Vector Potential \\
{$[C]$} & Aerodynamic Influence Coefficients \\
$C_{P}$ & Pressure Coefficient \\
$C_{1}, C_{2}$ & Attached and Separated Flow Coefficients \\
$D$ & Domain \\
$E A$ & Axial Stiffness \\
$E I$ & Flapwise and Edgewise Stiffness \\
$\epsilon$ & Vortex Particle Cutoff Length \\
$F_{S}$ & Local Normal Velocity of Deformations \\
$F_{L}$ & Load Operator \\
$F_{S}$ & Structural Equations of Motion \\
$\phi, s, \sigma, E, r, a$ & ONERA dynamic stall coefficients \\
& Velocity Scalar Potential \\
\hline & Kinematic Viscosity \\
\hline &
\end{tabular}




\begin{tabular}{|c|c|}
\hline$m$ & Mass Distribution \\
\hline$\mu$ & Dipole Intensity \\
\hline$n$ & Normal Vector \\
\hline$N$ & Number of Particles \\
\hline$P$ & A Reference Point in Space \\
\hline$\theta$ & Blade Pitch Angle \\
\hline$r$ & Distance to a Point \\
\hline$R$ & Vortex Particle Smoothing Function \\
\hline$\sigma$ & Source Intensity \\
\hline$t$ & Time \\
\hline$u$ & Velocity \\
\hline$v$ & Harmonic Vector Field \\
\hline$V_{\text {rot }}$ & Tangential Rotational Velocity \\
\hline$V_{\text {wind }}$ & Wind Velocity \\
\hline$\omega$ & Vorticity \\
\hline$\Omega$ & Vortex Intensity \\
\hline$X$ & Structural Variable Matrix \\
\hline$Z$ & Vortex Particle Position \\
\hline
\end{tabular}




\section{Chapter 1}

\section{Introduction}

\subsection{Motivation}

The study of aeroelasticity has many applications but two areas that are particularly pertinent to the aerospace industry are fixed-wing and rotary-wing applications. Aircraft wings are inherently flexible structures and are subject to a wide range of aeroelastic problems that affect their design and performance. An area that highlights the need for aeroelastic analysis is transonic flutter prediction. In the transonic flow regime, where most commercial aircraft cruise and many military aircraft operate, the influence of shockwaves and other nonlinear aerodynamics dictates the need for tools that can properly capture these effects and determine the flutter boundary of an aircraft. Aeroelastic rotary-wing applications include propellers, helicopters, and wind turbines. The prediction of wind turbine performance is dependent on the accuracy of both the structural and aerodynamic methods and is important to assess annual energy production in wind farms. A large amount of aeroelastic knowledge has been developed by the helicopter community and will be applied to study the aeroelastic behaviour of wind turbines. 


\subsubsection{Fixed-wing Aeroelasticity}

Fixed-wing aircraft flutter analysis is performed during the design and certification phases of aircraft development. Flutter analysis during the design phase can either be conducted using time domain methods or frequency domain methods. Time domain computational fluid dynamics (CFD) methods solve the Reynolds Averaged NavierStokes or Euler equations and can capture the nonlinear aerodynamics present in the transonic regime, but are computationally intensive. In time domain simulations, the structural dynamics model is coupled to the aerodynamic solver. As such, if the structural model changes throughout the design phase, the entire simulation set must be performed again. The calculation of the flutter point at a particular Mach number is also a trial and error method with time domain solvers as the flutter velocity must be determined by iterative simulations where one flow parameter is varied.

Frequency domain flutter analysis methods are linear solvers and cannot properly capture the nonlinear aerodynamics in transonic flow, but are very rapid and require only a few minutes of simulation time compared to several hours or days using time domain methods. In frequency domain methods, the aerodynamic and structural models are not closely coupled and so the structural model can be changed during the design process without affecting the matrices that describe the aerodynamic behaviour of the wing. In order to properly predict the flutter boundary in the transonic regime with frequency domain solvers, a method of correcting the aerodynamic matrices for nonlinear effects must be used.

Flutter certification is performed by flight testing a particular aircraft, a process which is both dangerous for the flight crew and expensive. It must be demonstrated during flight tests that the aircraft will not exceed the flutter boundary at any point within its flight envelope. The aircraft wing is excited in flight with an input signal and the damping behaviour of the wing is monitored at various flight conditions. 
The predicted flutter point is approached gradually at a risk to the flight crew if predictions were not accurate enough. The development of rapid, robust tools for the aircraft industry could help to reduce both design time and flight test time.

\subsubsection{Rotary-wing Aeroelasticity}

The prediction of the power output and performance of wind turbines is a research area that has seen growing interest recently as the demand for greater renewable energy production increases. As the power requirements of wind turbines has continued to increase, the average wind turbine blade radius has increased substantially from 7 $m$ in 1985 to almost $65 m$ in 2006. This substantial increase in rotor size is accompanied by large blade deflections in nominal operating conditions, as demonstrated in Figure 1.1 by the offshore turbines at the Horns Rev wind farm in Denmark. Most of the current aeroelastic wind turbine tools used for commercial and research applications still rely on linear models that assume small blade deflections. However, as the rotor size of wind turbines continues to increase, there is a need to develop more accurate structural models that can properly characterize the structural deformation of large turbines along with the accompanying turbine power output.

The power created by a turbine is also affected by the wake which will reduce the power generated by downstream turbines in a wind farm. The wake must be properly characterized in order to analyze the aerodynamic behaviour of turbines in normal and yawed flow, as well as in wind farms. The work in this thesis uses a nonlinear structural model coupled with a vortex particle potential flow method developed by the rotorcraft community and represents one of the few applications of such a model in the wind turbine field. 


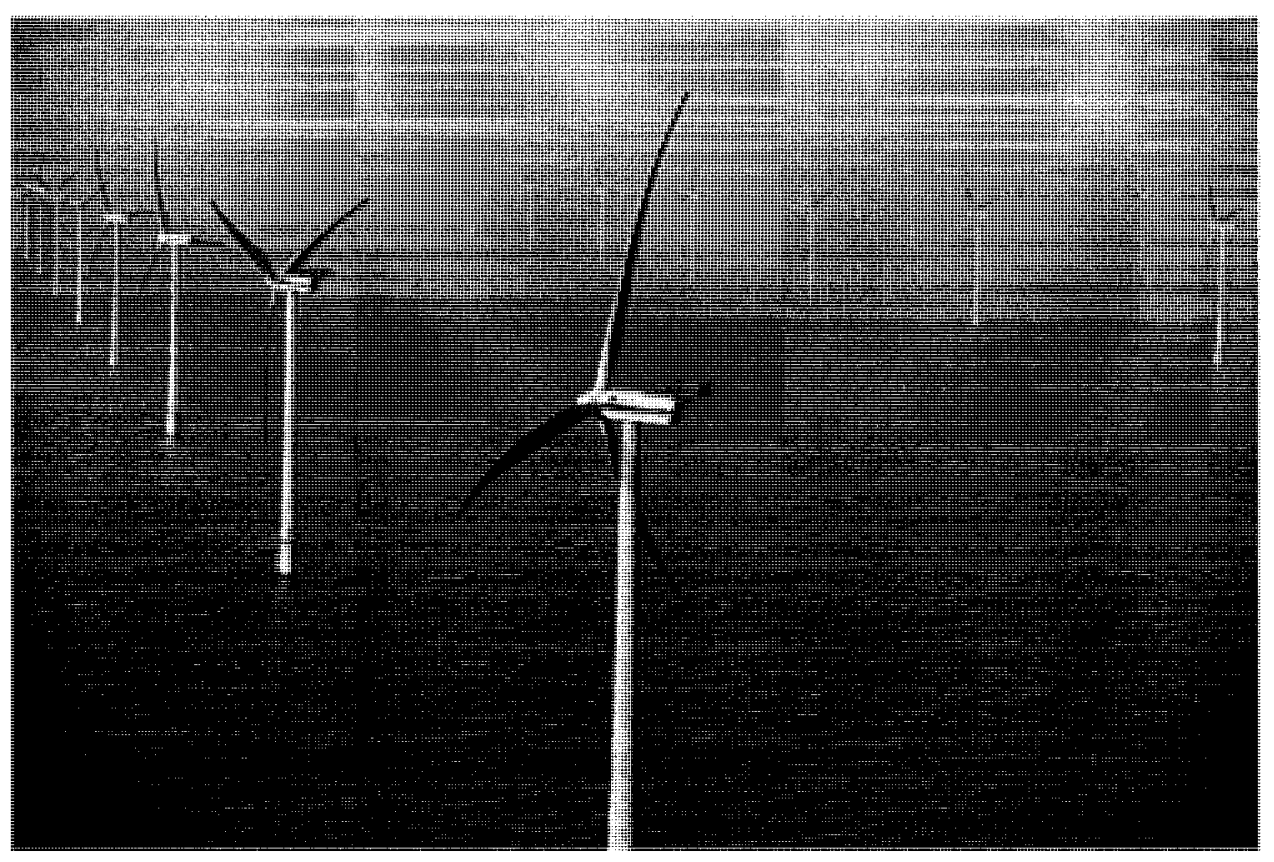

Figure 1.1: Deflection of the wind turbines at the Horns Rev wind farm.

\subsection{Objectives of the Thesis}

This thesis will address two seemingly distinct topics which are connected by the ambition to develop computational aeroelastic tools suitable for industrial applications. Fixed-wing transonic flutter and rotary-wing wind turbine applications will be both be addressed in this work. Tools that are suitable for industrial applications differ from research-based codes as they must be rapid, robust, and relatively simple to initialize. As such, aeroelastic CFD simulation time requirements should be less than one day and the grid generation requirements should be minimized whenever possible.

\subsection{Organization of the Thesis}

Due to the differences of the above two topics, it was decided to separate the thesis into two distinct parts. Part I will discuss the time domain and frequency domain tools applied to study the transonic flutter boundary of the AGARD 445.6 wing. 
The AGARD 445.6 wing was tested to flutter throughout the transonic regime in the NASA Transonic Dynamics Tunnel in 1963 and has become one of the three primary benchmark flutter experiments against which numerical codes are validated. The theory of the correction method developed for frequency domain flutter analysis along with the results of the aeroelastic time domain simulations have been previously discussed and presented by Beaubien [1]. As this work presents the corrected frequency domain simulations, the reader is referred to $[1,2]$ for a complete discussion of the background, theory, and equations describing both the time domain and frequency domain simulations. Only the essential theory and background along with the time domain CFD results and uncorrected frequency domain results from [1] will be described in this work for completeness.

Part II of the thesis discusses the use of SMARTROTOR, an aeroelastic and aeroacoustic analysis tool developed by the rotorcraft community at Carleton University [3], for studying wind turbine aerodynamics and wake behaviour. The historical development of wind turbines and a review of the computational techniques developed by and used by the wind turbine industry will be presented. The computational code SMARTROTOR is then validated against data from the National Renewable Energy Laboratory (NREL) full-scale wind turbine experiment. The $10 \mathrm{~m}$ diameter stallcontrolled NREL wind turbine was tested in the $24 \times 36$ m NASA Ames wind tunnel in 2000 and has become the benchmark experiment for code validation in the wind turbine industry since the results were published in 2002. Simulations were performed to assess the code's prediction of power output in unstalled, stalled, and yawed flow situations. SMARTROTOR's ability to perform wind farm wake interaction studies is also demonstrated. 


\section{Part I}

\section{Fixed-wing Aeroelasticity}




\section{Chapter 2}

\section{Time Domain and Frequency Domain Flutter Solutions}

\subsection{Description of Flutter}

Aircraft are flexible structures and as such, are susceptible to many aeroelastic phenomena including divergence, limit cycle oscillations, and flutter. Divergence is a static aeroelasticity instability in which aerodynamic loads can deform a wing to failure. Limit cycle oscillations (LCO) are periodic oscillations and can be self-excited or can be forced by a large disturbance. LCO will cause fatigue problems for certain components of an aircraft and can affect both passenger comfort and pilot endurance [4]. Flutter however, is an aeroelastic phenomenon that occurs due to undamped oscillations caused by structural and aerodynamic interactions at certain combinations of air density and freestream velocity. This dynamic instability can occur on control surfaces, wings, or fuselage sections, and can lead to catastrophic structural failure.

Before the flutter speed is reached, the amplitude of any disturbances to the wing

will be damped as seen in Figure 2.1(a). However, at a speed corresponding to the flutter boundary of an aircraft at a particular Mach number, the wing motion will resemble simple harmonic motion as demonstrated in Figure 2.1(b). If an aircraft is 
traveling faster than the flutter speed, any disturbances will not be damped and any oscillations of the wing will eventually lead to catastrophic failure as shown in Figure $2.1(\mathrm{c})$.

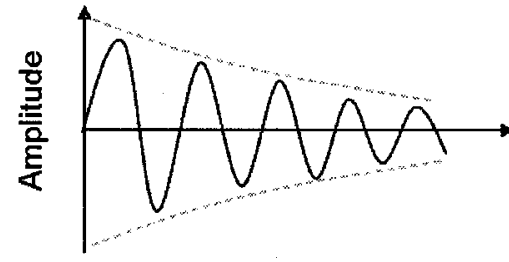

(a) Damped Oscillations.

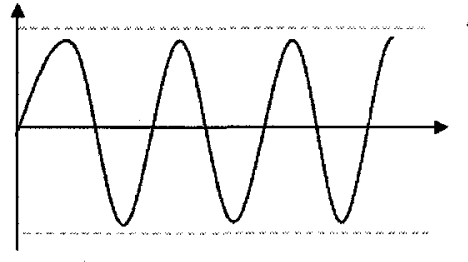

(b) Simple Harmonic Motion.

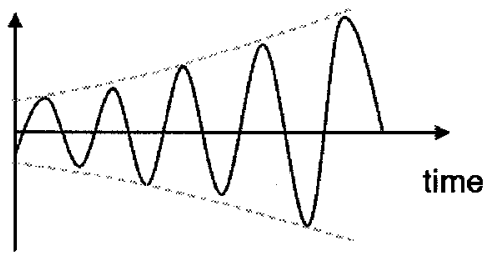

(c) Undamped Oscillations.

Figure 2.1: Amplitude response of a wing.

The first documented case of flutter occurred on the Handley Page 0/400 bomber in 1916 [4]. This incident involved dynamic twisting of the fuselage by $45^{\circ}$ in conjunction with antisymmetric flapping of the elevators [4]. On year after the 0/400 incident, tail flutter on a deHavilland DH-9 resulted in pilot fatalities [5]. Following the First World War, an experimental and theoretical study of flutter was conducted in the Netherlands after aileron flutter occurred on a van Berkel W.B. reconnaissance seaplane. Theoretical work concerning flutter followed shortly in the United Kingdom and in the United States of America. Theodorson made significant contributions to flutter theory in the 1930s with the development of two-dimensional flutter theory [5]. Throughout the Second World War, as aircraft attained higher flight speeds, the instances of flutter increased significantly, and there were over 50 instances of flutter in the 10 years that followed the War. Transonic flutter became an issue with the development of the turbojet engine and presented a wide range of new problems [5]. Due to the development of computational techniques and improved wind tunnel testing, the number of flutter incidents were drastically reduced by the 1970s. While the few instances of flutter in the past ten years have occurred on military jets [1], a very recent instance of flutter occurred in 2006 during the flight testing of a Grob Aerospace SPn business jet aircraft. Elevator flutter caused partial separation of the 
elevator and horizontal stabilizer and the test aircraft crashed [6]. Though the number of flutter incidents has decreased substantially, all aircraft must be certified for flutter and other aeroelastic phenomena.

During certification, commercial and military aircraft must demonstrate through wind tunnel testing, numerical simulations, and flight testing that the aircraft will not experience flutter within a boundary $15 \%$ larger than the flight envelope of the aircraft [7]. A representative schematic describing the bending-torsion flutter boundary and the flight envelope of an aircraft can be seen in Figure 2.2. The flutter boundary defines the speed at which the onset of flutter can occur due to a perturbation of the wing and aircraft must fly below the flutter speed at all Mach numbers. The regions of the flight envelope where the damped, harmonic, and undamped motions presented above in Figure 2.1 will occur are indicated in Figure 2.2 by the letters $a, b$, and $c$, respectively. The most critical region of the flutter boundary is characterized by the transonic dip and typically occurs between a Mach number of 0.9 and 1.1. After a Mach number of 1.2, the flutter speed rises due to the rearward shift in the centre of pressure on the surface of the wing and is typically not a concern. However, in the most critical region between Mach numbers of 0.9 and 1.1, nonlinear transonic effects are present over the wing and control surfaces of aircraft that cannot be properly captured by linear solution techniques.

These transonic effects include the location and strength of shockwaves over the surface of the wing and the associated shockwave-boundary layer interactions, as seen in Figure 2.3. Figure 2.3(a) depicts the pressure contours over the surface of a wing in transonic flow. The shocks that occur over the surface are located at the dark concentrations of the pressure contours. Figure 2.3(b) represents a crosssectional view of the wing presented in 2.3 (a) and demonstrates that there will be a region of supersonic flow over the upper or lower surfaces of the wing in transonic flow, terminating in a normal shock. The location and strength of the shock varies 


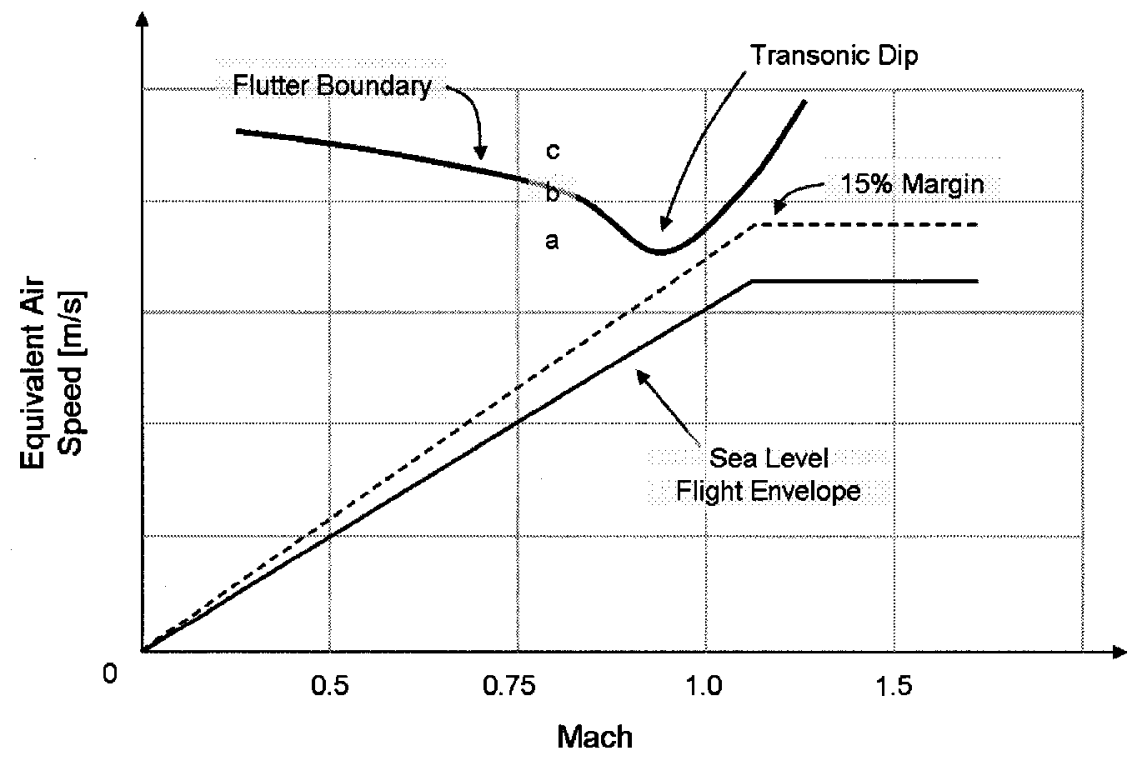

Figure 2.2: Schematic of the Flutter Boundary of an Aircraft. Adapted from [7].

along the span of a wing, depends on the velocity and density of the flowfield, and moves toward the leading edge or trailing edge of a wing due to oscillations and deflection of the wing. The shockwave-boundary layer interaction will also promote flow separation over the wing. These nonlinear phenomena are responsible for the characteristic transonic dip in the flutter boundary that occurs between the Mach numbers of 0.7 and 1.1 and must be properly predicted in order to assess the flutter boundary in the transonic flow regime. Time domain Reynolds-Averaged NavierStokes CFD simulations can inherently predict these effects in transonic flow and can accurately determine the flutter boundary at these flight speeds. Conversely, uncorrected linear frequency domain simulations cannot predict these phenomena and therefore aerodynamic correction techniques must be developed to accurately predict the flutter boundary in the frequency domain.

The response outputs from time domain and frequency domain flutter simulations are quite different and are demonstrated in Figure 2.4. Time domain flutter analysis is performed iteratively by varying one flow parameter (typically density) 


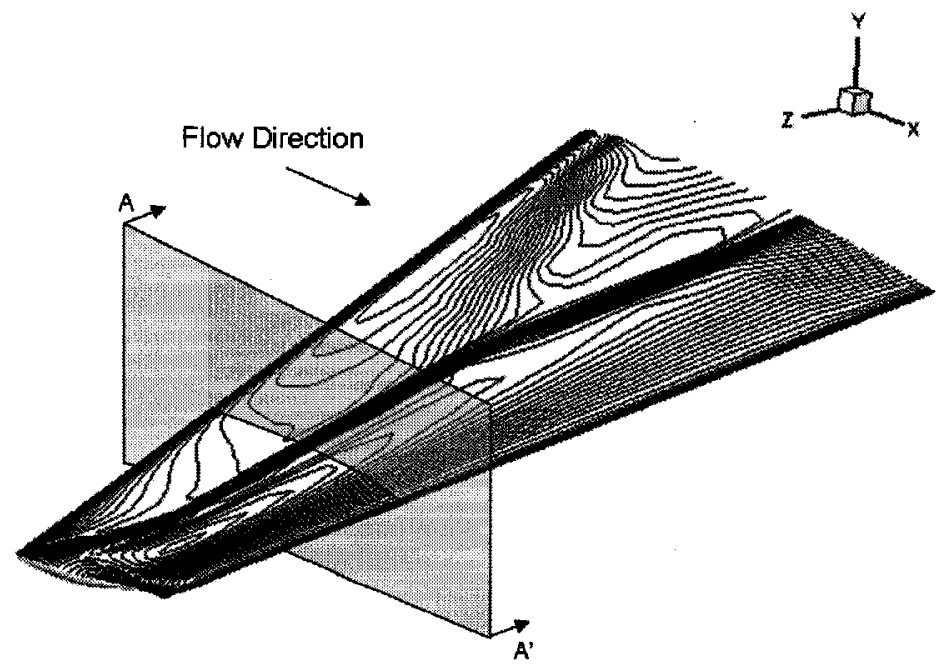

(a) Pressure contours on a wing in transonic flow.

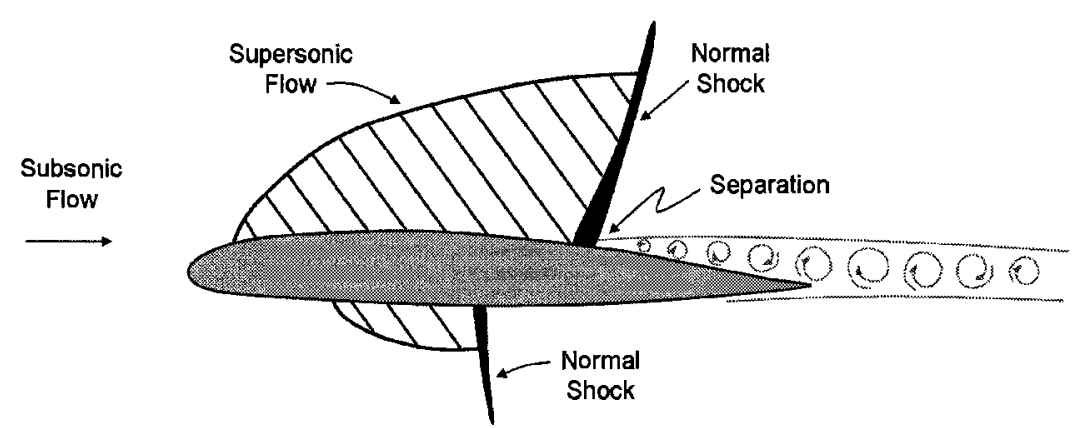

(b) Schematic of the nonlinear effects at a wing cross section A-A.

Figure 2.3: Nonlinear effects over a wing in transonic flow.

and monitoring the oscillatory response of a simulation that has been provided with an initial perturbation. This is demonstrated in Figure 2.4(a) where the amplitude response of the first four modes of a wing are monitored in time over three cycles at two different air densities. The flutter velocity is found at the density where simple harmonic motion is recovered. In frequency domain simulations however, the output is composed of velocity-frequency and velocity-damping plots that demonstrate the two modes that coalesce to produce flutter and the flutter speed, respectively. This is demonstrated in Figure 2.4(b) where the flutter speed occurs at a value of zero 
damping when simple harmonic motion of the wing occurs.

In this work, time domain CFD simulations, uncorrected frequency domain simulations, and corrected frequency domain simulations of a wing throughout the transonic flow regime are presented and validated with a benchmark experimental test case.

\subsection{Time Domain Analysis}

Time domain flutter simulations are more accurate, however they are complex simulations to perform and are time consuming, requiring approximately one day to determine a single flutter point. Time domain flutter simulations were first successfully conducted about 25 years ago and are not presently used at the industrial level for design purposes. The advantage of using time domain CFD is that the nonlinear effects present in transonic flow can be more accurately captured.

One of the first computational flutter simulations was performed in 1982 by Borland et al. where the loosely coupled code XTRANS3S was applied to a rectangular wing [8]. The fluid solution was modeled with the Transonic Small Disturbance (TSD) equations and the structure of the wing was modeled by the modes of the structure. Flutter analysis with the TSD equations was later extended in 1988 by Cunningham et al. [9] who attempted to predict the flutter boundary of the AGARD 445.6 wing using the CAP-TSD code. One of the original applications of a three-dimensional Euler CFD solution to the flutter boundary of a wing was given by Rausch et al. first in 1993 [10] using an unstructured grid and later in 1995 using a structured grid [11]. In 1999, a loosely coupled code was developed by Goura [12] and was used for flutter analysis using the Euler flow model with a finite element beam representation of the structure [12]. More recently, in 2003, the Navier-Stokes equations were used with a detailed finite element model to perform aeroelastic CFD simulations of a complete F-16 aircraft [13]. 

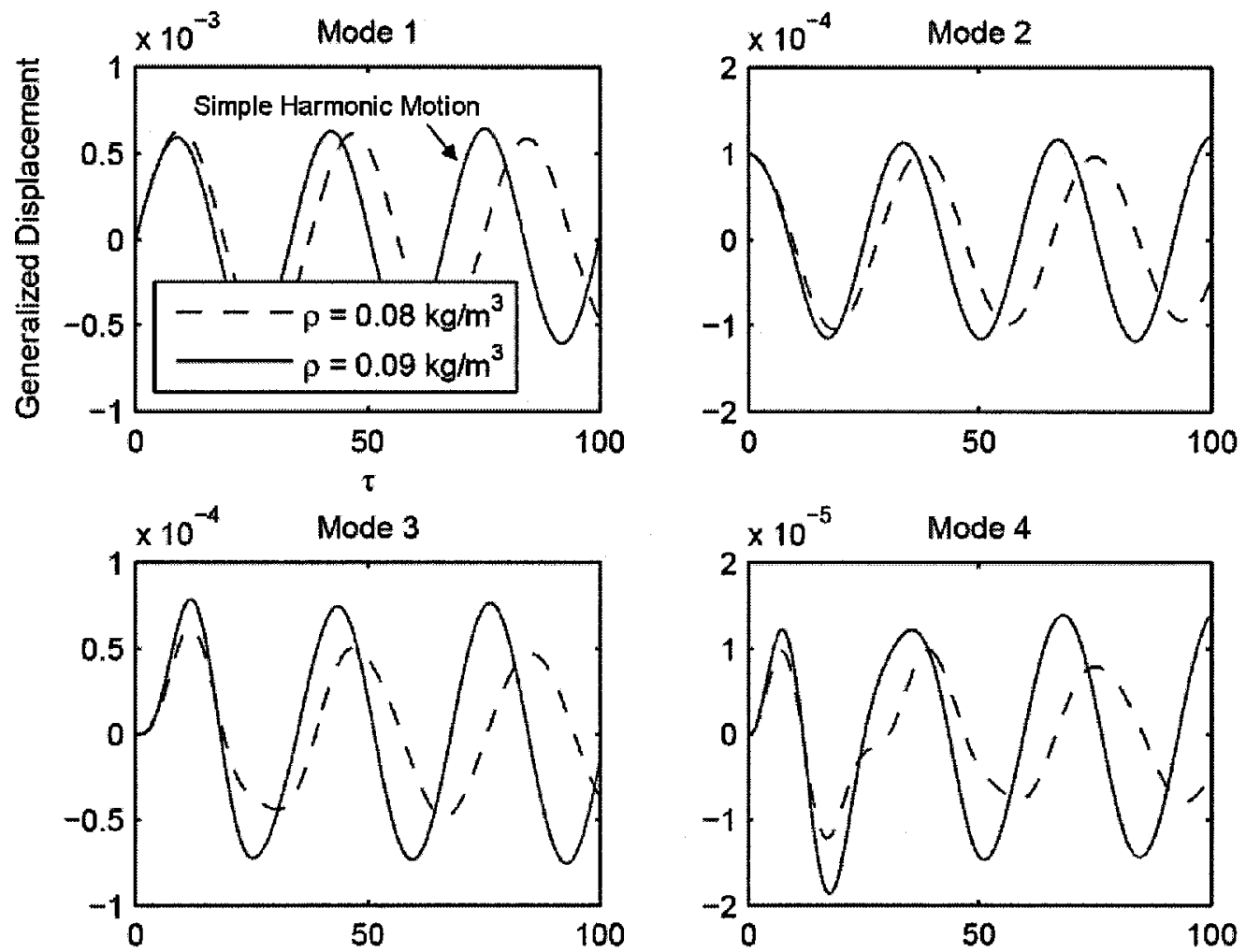

(a) Time domain response - Navier-Stokes [1].
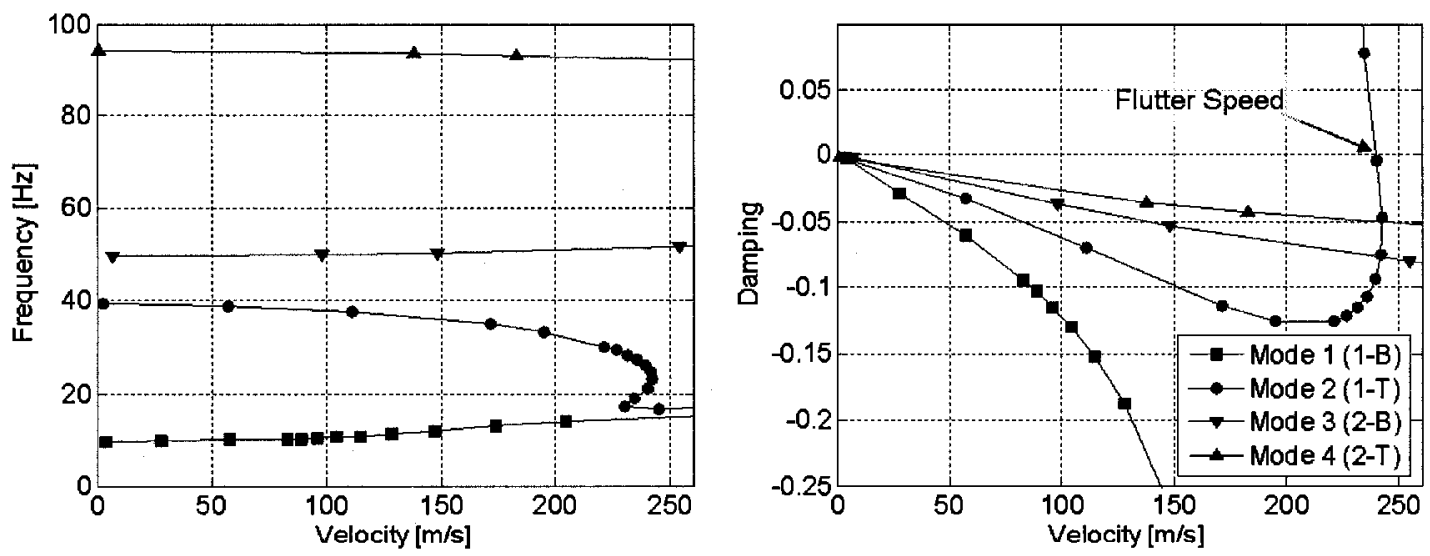

(b) Frequency domain response - NASTRAN

Figure 2.4: Output from time domain and frequency domain methods used to calculate a single flutter point. 
In the current work, the time domain flutter simulations were conducted using both Euler and Navier-Stokes representations [1] and will serve as the benchmark case for the corrected frequency domain technique, along with the experimental results from the AGARD 445.6 wind tunnel test.

\section{Time Domain Aerodynamic Model}

The time domain flutter simulations were performed with the Carleton Multi-Block (CMB) Computational Fluid Dynamics code which is based on a code originally developed at the University of Glasgow [14] and further extended at Carleton University. $\mathrm{CMB}$ has a proven capability of capturing transonic effects $[12,14]$. It is a three-dimensional CFD code that uses a cell-centred finite volume technique to solve either the Euler or the Reynolds Averaged Navier-Stokes (RANS) equations. The diffusive terms are discretized with a central differencing scheme and the convective terms are solved using an approximate Riemann solver that applies either Roe's or Osher's scheme. Osher's upwind scheme was used in all of the simulations performed as part of this study. Second order spatial accuracy is achieved with MUSCL variable extrapolation with a Van Albada limiter to prevent dispersion near shockwaves [1].

The boundary conditions are dealt with using two layers of ghost cells that lie outside the domain. The far field boundary condition is met by setting the ghost cell values equal to the freestream values. For viscous flows, no-slip is imposed at the wall and for inviscid flows, the normal velocity on any solid surfaces is set to zero.

Steady flow simulations begin with an explicit scheme to smooth out the flow solution, which then switches to an implicit scheme for more rapid convergence. Preconditioning is based on block incomplete lower-upper surface factorization and is decoupled between blocks to increase the parallel performance of the solver [15]. In unsteady flows, the multigrid dual-time method of Jameson is used to obtain second order accuracy in time. In the Jameson dual-time method, equations are solved 
implicitly in both pseudo-time and real-time.

Turbulence modeling in CMB can be performed using a variety of models, including the Spalart-Allmaras, $\mathrm{k}-\epsilon, \mathrm{k}-\omega$, and Shear Stress Transport $(S S T)$ models. The two-equation baseline $S S T$ model was selected for this study because of its applicability to simulations with complex surface interactions and based on other tests performed with CMB [16]. The SST model blends the $\mathrm{k}-\epsilon$ model (which is generally robust outside the boundary layer) with the $\mathrm{k}-\omega$ model (which is more robust within the boundary layer). A complete description of the $S S T$ model that was implemented in this study can be found in $[1,16]$.

\section{Time Domain Structural Model}

In order to accurately model aeroelastic problems, the geometry of the wing and the aerodynamic mesh must both deform during simulations. The static and dynamic response of the structure is resolved using a finite element method linear beam model. The mass $([M])$ and stiffness $([K])$ matrices of an elastic wing are related to external forces $\left(\left\{f_{S}\right\}\right)$ according to Equation 2.1

$$
[M]\{\ddot{u}\}+[K]\{u\}=\left\{f_{S}\right\}
$$

The mass and stiffness matrices in the linear structural system were determined a priori with MSC/NASTRAN.

The structural displacements are written as a linear combination of the generalized coordinates $(\{q\})$ and the physical displacements of the structure $(\{u\})$ according to Equation $2.2[1]$

$$
\{u\}=[\Phi]\{q\}
$$

where $[\Phi]$ is the modal transformation matrix. The eigenvalues of the system are 
solved and scaled according to Equation 2.3.

$$
[\Phi]^{T}[M][\Phi]=1
$$

The finite element method equations can then be described by Equation 2.4 .

$$
\frac{d^{2}\{q\}}{d t^{2}}+\omega^{2}\{q\}=[\Phi]^{T}\left\{f_{S}\right\}
$$

Equation 2.4 is solved using a two-step Runge-Kutta method and is iterated in pseudo time along with the aerodynamic solution to avoid time sequencing errors from the dual-time approach [17].

For aeroelastic simulations, the deformation of the computational domain is accomplished by interpolating the wing surface displacements to the interior nodes. The deformation of the aerodynamic mesh is determined in conjunction with the structural beam model deflection using Transfinite Interpolation of Displacements (TFI) within all of the blocks containing a wing surface [17]. The wing surface deflections $\left(x_{a, i k}\right)$ are interpolated to the grid points within the domain $\left(x_{i j k}\right)$ according to Equation 2.5 .

$$
\delta x_{i j k}=\varphi_{j}^{0} x_{a, i k}
$$

The term $\varphi_{j}^{0}$ in Equation 2.5 represents a blending function which varies between one at the wing surface and zero at the opposite face of the block containing the wing surface. The cell volumes are recalculated using a Global Conservation Law (GCL) by considering volume fluxes through the cell faces. The deflections of the wing surface $\left(x_{a, i k}\right)$ are obtained from the transformation of the structural grid deflections [1].

As the structural and aerodynamic meshes are not coincident in this type of analysis, coupling between the structural and aerodynamic models is achieved using the Constant Volume Tetrahedron (CVT) scheme [17]. 
A complete description of the CMB code can be found in [12] and [14].

\subsection{Uncorrected Frequency Domain Analysis}

In frequency domain flutter analysis, the equations of motion are linearized and transformed into the frequency domain. The aerodynamic forces are computed with a potential-flow approach and are related to structural deformations using an aerodynamic influence coefficients (AIC) matrix [1]. The roots of the equations of motion are determined using a variety of eigenvalue techniques. Roots that correspond to decaying conditions are below the flutter speed, whereas roots that correspond to diverging conditions are above the flutter point. Roots that correspond to neutrally stable conditions (simple harmonic motion) represent the flutter point at a particular Mach number.

The current industry-standard subsonic flutter analysis technique is based on the Doublet-Lattice Method (DLM) linearized aerodynamic theory. The DLM was developed by Albano and Rodden in the 1960s [18] and is a commonly used unsteady potential flow technique that is available in commercial frequency domain flutter analysis software such as MSC/NASTRAN. [7]. The DLM uses linearized aerodynamics that are only strictly valid in the subsonic flow regime and fails to capture the previously described nonlinear effects present in transonic flow [1].

In order to account for nonlinear transonic effects with the DLM, the AIC matrix must be corrected with one of four currently-used methods [1]. These include force matching, pressure matching, Dau-garner, and modal AIC matrix replacement methods. Force matching methods match nonlinear forces and moments that can be obtained with CFD simulations whereas pressure matching methods match reference nonlinear pressures. In the two preceding methods, nonlinear effects are accounted for using the force or pressure reference conditions from CFD simulations and are 
incorporated into a corrected AIC matrix. Dau-garner methods use steady nonlinear data and semi-empirical relationships to determine corrected nonlinear pressures. The modal AIC method however, creates a new nonlinear AIC matrix that is based on the modal displacement of a wing [1].

The current frequency domain method uses the pressure matching method developed by Reference [19] to correct the AIC matrix and will be presented in Section 2.4. A thorough discussion of the above-mentioned correction methods can be found in $[1,19]$.

The uncorrected aerodynamic modeling with the DLM and the representation of the aeroelastic system of equations in the frequency domain will be briefly presented in the following Section, but a more detailed derivation of the relevant equations and a description of the interconnection of the structure with the system aerodynamics is given by Beaubien [1]. The derivation of the selected correction method to account for nonlinear aerodynamics in the frequency domain is then presented.

\subsubsection{Aerodynamic Modeling}

Lifting surface theory is used to solve the unsteady compressible flow over a wing using the Doublet Lattice Method (DLM) assuming that the flow is inviscid, isentropic, subsonic, and fully attached. The thickness of the lifting surface is neglected and small-perturbation theory is used assuming that changes in the angle of attack will be small. Compressibility is taken into account using the Prandtl-Glauert formula [20]. The DLM is based on a panel method approach and is widely used in industry. While the following is an introduction to the DLM, a detailed derivation can be found in $[21,18]$.

The linearized oscillatory subsonic lifting surface theory relates the normal velocity $(W)$ at the lifting surface to the pressure coefficient difference over the surface $\left(\Delta C_{P}\right)$, 
which are both defined in Equations 2.6 and 2.7.

$$
W=U_{\infty} \operatorname{Re}\left(w e^{i \omega t}\right)
$$

$$
\Delta P=1 / 2 \rho U_{\infty}^{2} \operatorname{Re}\left(\Delta C_{P} e^{i \omega t}\right)
$$

The normal velocity and pressure difference can be related by an integral equation written in the reduced frequency domain, as described by Equation 2.8

$$
w(x, y, z)=\frac{1}{8 \pi} \iint K\left(x-\xi, y-\eta, z-\zeta, k, M_{\infty}\right) \Delta C_{P} d \xi d \sigma
$$

where $\eta$ is the streamwise coordinate, $\sigma$ is the tangential spanwise coordinate, $k=w b_{s} / U_{\infty}$ is the reduced frequency, $\omega$ is the oscillation frequency, $b_{s}$ is the root semi-chord, and $U_{\infty}$ is the freestream velocity. The kernel $K$ in Equation 2.8 is the downwash at a point $(x, y, z)$ induced by a doublet of unit strength located at $(\xi, \eta, \zeta)$ in elemental coordinates [22]. The lifting surface is divided into a grid of trapezoidal elements over which the pressure is constant [1]. The double integral over one element can be performed in the $\xi$ and $\bar{\eta}$ directions. The integration in the streamwise $\xi$ direction by concentrating the integrand at the quarter-chord point of a panel, $\eta_{1 / 4}$ and Equation 2.8 can be rewritten as

$$
w(x, y, z)=\sum_{j} \Delta C_{P} \frac{\Delta \xi_{j}}{8 \pi} \int_{-e_{j}}^{e_{j}} K\left(x-\xi_{1 / 4}, \bar{y}-\bar{\eta}, \bar{z}, k, M_{\infty}\right) d \bar{\eta}
$$

where $e$ is the semi-width of a lifting panel. The downwash boundary condition in Equation 2.9 is known and the pressure difference coefficient over each element is unknown. If the downwash can be satisfied for each element in the lifting surface, 
Equation 2.9 can be written in matrix form as Equation 2.10.

$$
\{w\}=[D]\left\{\Delta C_{P}\right\}
$$

Each element in $[D]$ is a downwash factor $D_{i j}$ at the location $x_{i}, \bar{y}_{i}, \bar{z}_{i}$ caused by element $j$ as seen in Equation 2.10.

$$
D_{i j}=\frac{\Delta \xi_{j}}{8 \pi} \int_{-e_{j}}^{e_{j}} K\left(x_{i}-\xi_{1 / 4}, \bar{y}_{i}-\bar{\eta}, \bar{z}_{i}, k, M_{\infty}\right) d \bar{\eta}
$$

The downwash factor $D_{i j}$ can be separated into a steady and unsteady part and the solution must satisfy both the Kutta condition and the flow tangency condition. The Kutta condition states that the pressure difference at the trailing edge of a thin lifting surface must be zero and the flow tangency condition states that the velocity vector at a surface must be tangent to the surface of a lifting body in inviscid flow [23]. The flow tangency condition is satisfied at the control and receiving point found on each element. The downwash collocation control point is centred along the three-quarter chord line of each element [1].

The pressure distribution in Equation 2.10 can be obtained by multiplying the inverse of the downwash factor matrix $[D]$ by the downwash vector $\{w\}$. The inverse of $[D]$ is the AIC matrix and can be related to the pressure coefficient as a function of reduced frequency according to Equation 2.12. The coefficients of the AIC matrix represent rates of the pressure variation caused by a particular displacement amplitude input [1]. The downwash vector in Equation 2.12 is related to the amplitude of the pitch and plunge motions at each panel.

$$
\left\{\Delta C_{P}(i k)\right\}=[A I C(i k)]\{w(i k)\}
$$

Once the pressure coefficients have been determined, the aerodynamic loading vector 
$\left(\left\{L_{a}(i k)\right\}\right)$ can be expressed as Equation 2.13, in which $[S]$ is an integration matrix that depends on the lifting panel geometry $[19],[F(i k)]$ is the substantial derivative applied to a modal displacement $\{h\}$, and $q_{\infty}$ is the dynamic pressure.

$$
\left\{L_{a}(i k)\right\}=q_{\infty}[S][A I C(i k)][F(i k)]\{h\}
$$

\subsubsection{Aeroelastic Modeling}

The equation of motion for a lifting body in an airstream can be written as the equilibrium between the structural and aerodynamic forces, as seen in Equation 2.14.

$$
[M]\{\ddot{u}(t)\}+[K]\{u(t)\}=\{L(u(t), \dot{u}(t))\}
$$

In Equation 2.14, $[M]$ and $[K]$ are the structural mass and stiffness matrices, respectively and are obtained from a finite element model of the lifting surface [1]. The structural deformation can be written as $\{u\}$ and the acceleration of the body can be written as $\{\ddot{u}\}$. The forces $[M]\{\ddot{u}(t)\}$ and $[K]\{u(t)\}$ in Equation 2.14 represent the inertial and elastic structural forces, respectively, and the structural damping force is neglected in this derivation. The aerodynamic forces $\{L\}$ in Equation 2.14 are composed of two components: the aerodynamic forces induced by the structural deformation, $\left\{L_{a}(u(t), \dot{u}(t))\right\}$ and the external forces, $\left\{L_{e}(t)\right\}$. In the presence of ex-

ternal forces (such as gusts, turbulence, control surface forces), Equation 2.14 can be written as an aerodynamic feedback system [19] according to Equation 2.15.

$$
[M]\{\ddot{u}(t)\}+[K]\{u(t)\}-\left\{L_{a}(u(t), \dot{u}(t))\right\}=\left\{L_{e}(t)\right\}
$$

The flutter boundary is determined by investigating the dynamic stability of Equation 2.15. The flutter boundary represents the structural stability boundary of an aircraft 
as a function of dynamic pressure and can be found by examining the structural response at a particular flight condition [1]. Because the amplitudes of deformations are small for aeroelastic phenomena [20], the structural dynamics model can be considered to be linear. As such, Equation 2.15 is a linear system and the flutter boundary can be determined by solving the complex eigenvalues of the system. The solution of the eigenvalues of the system require rewriting the homogeneous aeroelastic system of equations (in the absence of external forces) described by Equation 2.16 in the Laplace domain.

$$
[M]\{\ddot{u}(t)\}+[K]\{u(t)\}-\left\{L_{a}(u(t), \dot{u}(t))\right\}=0
$$

The induced aerodynamic forces are transformed into the Laplace domain with a convolution integral [1] and the transformed loading in the Laplace domain is described by Equation 2.17

$$
\left\{L_{a}(u(s))\right\}=q_{\infty}\left[\bar{H}\left(\frac{s b}{U_{\infty}}\right)\right]\{u(s)\}
$$

where $\bar{H}$ is the aerodynamic transfer function in the Laplace domain and $\left(s b / U_{\infty}\right)$ is the non-dimensional Laplace variable. The remainder of Equation 2.16 is transformed into the Laplace domain and the resulting eigenvalue problem is expressed in Equation 2.18 .

$$
\left(s^{2}[M]+[K]-q_{\infty}\left[\bar{H}\left(\frac{s b}{U_{\infty}}\right)\right]\right)\{u(s)\}=0
$$

\section{Modal Analysis}

The direct solution of Equation 2.18 is computationally intensive as the finite element model of an aircraft structure contains a large number of degrees of freedom [1]. As such, the structural dynamics system is transformed to modal space, where the resulting eigenvalues represent the natural frequencies of the system and the eigenvectors are the associated mode shape vectors. The structural displacements in modal space 
are a linear combination of the generalized coordinates $\{q(t)\}$ and the displacements of the structure $\{u(t)\}$ according to Equation 2.19.

$$
\{u(t)\}=[\Phi]\{q(t)\}
$$

The modal transformation matrix $[\Phi]$ in Equation 2.19 is composed of columns containing the mode shape vectors, which are each associated to a particular natural frequency of the structure. As the critical flutter modes occur due to the coupling of lower order structural modes, the structural deformation of the flutter mode can be represented by the superposition of lower order modes [1]. The aeroelastic system previously described by Equation 2.18 can be written in modal space as

$$
\left(s^{2}[\bar{M}]+[\bar{K}]-q_{\infty}\left[Q\left(\frac{s b}{U_{\infty}}\right)\right]\right)\{q(s)\}=0
$$

where $\{q(s)\}$ is the generalized coordinate in the Laplace domain and $[\bar{M}],[\bar{K}]$, and $\left[Q\left(s b / U_{\infty}\right)\right]$ are the generalized mass, stiffness, and aerodynamic force matrices which can be expressed in Equations 2.21, 2.22, and 2.23 .

$$
\begin{gathered}
{[\bar{M}]=[\Phi]^{T}[M][\Phi]} \\
{[\bar{K}]=[\Phi]^{T}[K][\Phi]} \\
{\left[Q\left(\frac{s b}{U_{\infty}}\right)\right]=[\Phi]^{T}\left[\bar{H}\left(\frac{s b}{U_{\infty}}\right)\right][\Phi]}
\end{gathered}
$$




\section{Simple Harmonic Motion Approach}

As the computation of the aerodynamic transfer functions in the Laplace domain can be difficult, unsteady aerodynamic methods can be formulated in the frequency domain by assuming simple harmonic motion [1]. The aerodynamic transform function in the simple harmonic reduced frequency domain is the Aerodynamic Inflence Coefficient (AIC) matrix [1]. As the AIC matrix is only available in this domain, the imaginary component of the aeroelastic system of equations described by Equation 2.20 is given by Equation 2.24 where $s$ is replaced by $i w$.

$$
\left(-\omega^{2}[\bar{M}]+[\bar{K}]-q_{\infty}[Q(i k)]\right)\{q(i \omega)\}=0
$$

\section{Flutter Analysis Techniques}

The flutter behaviour of a wing is analysed by evaluating the stability of an aeroelastic system. The three industry-standard flutter techniques are the American $K$-method, the more efficient $K E$-method, and the British $P K$-method. In the $K$-method, the aerodynamic loads are treated as complex masses and the method determines the frequencies and damping required to sustain simple harmonic motion. The structural damping in the $K$-method is treated by a matrix.

The $K E$-method is very similar to the $K$-method but is significantly faster due to the fact that no damping matrix is included and no eigenvector recovery is made. Structural damping effects can be included, but are treated as part of the complex stiffness matrix. In both the $K$ - and $K E$-methods, the artificial structural damping is a mathematical term that does not represent the physical damping behaviour of the system. Both methods however, can accurately predict the flutter speed, which occurs when simple harmonic motion is recovered at zero damping.

The $P K$-method has the ability to determine the stability of the system and to 
estimate the physical damping of the system at subcritical speeds (at speeds below the flutter boundary). The aerodynamic matrices are treated as frequency dependent springs and dampers in the $P K$-method.

The frequency domain flutter analysis was performed using MSC/NASTRAN and KEDLMPL. MSC/NASTRAN is an industry-standard finite element package that can perform $K, K E$, and $P K$ flutter analyses. The software was rewritten in 2001, and as such, the aerodynamic matrices are no longer accessible for interpretation or correction [1]. The second software package that was used is KEDLMPL, a frequency domain flutter analysis tool designed at the Instituto de Aeronáutica e Espaço in Brazil [19]. As the source code for KEDLMPL was generously provided, the aerodynamic matrices were accessible, and aerodynamic corrections could be implemented and evaluated. As KEDLMPL was designed specifically to perform $K E$-flutter analysis for wings, all of the frequency domain flutter analyses with KEDLMPL and MSC/NASTRAN were performed with the $K E$-method for consistency between frequency domain results.

\subsection{Corrected Frequency Domain Analysis}

A procedure was developed to correct the frequency domain flutter boundary produced with KEDLMPL to account for nonlinear aerodynamics in the transonic regime [1]. The technique corrects the Aerodynamic Influence Coefficients (AIC) matrix used within the DLM solver. A pressure matching method was applied that involved a downwash weighting approach using unsteady nonlinear pressure coefficients obtained from time domain CFD simulations $[1,19]$. The relationship between the AIC matrix, the downwash vector $(w)$, and the pressure difference coefficient vector $\left(\Delta C_{P}\right)$ is described in Equation 2.25 as a function of reduced frequency. The uncorrected AIC matrix is computed within the DLM and relates the lifting pressures 
to a given displacement input and is independent of the vibration modes.

$$
\left\{\Delta C_{P}(i k)\right\}=[A I C(i k)]\{w(i k)\}
$$

The nonlinear unsteady lifting pressures are obtained by performing unsteady rigid body pitching motions with CFD. Based on the work of [19], a harmonic pitching motion about the root midchord was simulated in CMB according to Equation 2.26

$$
\alpha(t)=\alpha_{m}+\Delta \alpha \sin \left(2 k_{r} t\right)
$$

where the mean angle of attack $\left(\alpha_{m}\right)$ was $0^{\circ}$, the amplitude of the oscillation $(\Delta \alpha)$ was $1^{\circ}$, and $k_{r}$ represents the reduced frequency of the oscillatory motion. The parameters used for the unsteady pitching motion were based on the experiences of Silva [19]. Following the harmonic pitching simulation, the pressures obtained on the upper and lower surface of the wing over one complete pitching cycle are subtracted to obtain the nonlinear $\Delta C_{P}$ vector, as described in Equation 2.27

$$
\left\{\Delta C_{P}^{N L}\right\}=C_{P}^{L O W E R}-C_{P}^{U P P E R}
$$

As the AIC correction procedure is implemented in the frequency domain, the $\Delta C_{P}$ vector described in Equation 2.27 must be Fourier transformed to obtain the frequency domain representation of the unsteady lifting pressures. The first harmonic of the unsteady pressure coefficients was obtained using the discrete Fourier transform (DFT) described by Equations 2.28 and $2.29[24,19]$.

$$
\operatorname{Re}\left(C_{P}^{N L}\right)=\frac{k_{r} M \Delta \tau}{2 \pi \Delta \alpha} \sum_{m_{1}}^{m_{1}+m_{\tau}} C_{P} \sin \left(k_{r} m \Delta \tau\right)
$$




$$
\operatorname{Im}\left(C_{P}^{N L}\right)=\frac{k_{r} M \Delta \tau}{2 \pi \Delta \alpha} \sum_{m_{1}}^{m_{1}+m_{\tau}} C_{P} \cos \left(k_{r} m \Delta \tau\right)
$$

where $m$ is the time step number, $M$ is the Mach number of the unsteady simulation, and $\Delta \tau$ is the non-dimensional time step. A complete derivation of the DFT can be found in Appendix A.

The nonlinear lifting pressure vector is then written in complex notation according to Equation 2.30 prior to its implementation.

$$
\left\{\Delta C_{P}^{N L}\right\}=\left(\operatorname{Re}\left\{\Delta C_{P}^{N L}(i k)\right\}+\operatorname{Im}\left\{\Delta C_{P}^{N L}(i k)\right\}\right)
$$

In order to account for nonlinear aerodynamics in the AIC matrix, Equation 2.25 is modified to include a nonlinear weighting matrix $[W T]$, as seen in Equation 2.31. The normalized downwash vector term $\left(w^{\alpha}\right)$ in Equation 2.31 is normalized by the amplitude of the unsteady pitching motion as described in Equation 2.32 [19].

$$
\begin{gathered}
\left\{\Delta C_{P}^{N L}\right\}=[A I C(i k)][W T(i k)]\left\{w^{\alpha}(i k)\right\} \\
\left\{w^{\alpha}(i k)\right\}=\frac{\{w(i k)\}}{\Delta \alpha}
\end{gathered}
$$

Prior to solving the weighting matrix, a nonlinear downwash term must be calculated based on the nonlinear lifting pressures. The nonlinear downwash equation is obtained by multiplying the Fourier transformed nonlinear pressures from the unsteady rigid body CFD simulation with the AIC matrix by rearranging Equation 2.25 below.

$$
\left\{w^{N L}(i k)\right\}=[A I C(i k)]^{-1}\left\{\Delta C_{P}^{N L}\right\}
$$

The nonlinear downwash vector is then divided by the normalized downwash vector 
from Equation 2.32 to produce the nonlinear weighting matrix $[W T]$, as seen in Equation 2.34 [19].

$$
[W T(i k)]=\frac{\left\{w^{N L}(i k)\right\}}{\left\{w^{\alpha}(i k)\right\}}
$$

Once the weighting matrix has been determined, the nonlinear aerodynamic loads can be calculated using Equation 2.35, where $q_{\infty}$ is the dynamic pressure and $[S]$ is an integration matrix constructed from the geometric characteristics of the lifting panel [1]. The nonlinear aerodynamic loads $\left(L_{a}^{N L}\right)$ are used in the frequency domain solver in order to calculate the corrected frequency domain flutter boundary. Additional information regarding the AIC correction procedure can be found in Reference [19].

$$
L_{a}^{N L}=q_{\infty}[S][A I C(i k)][W T(i k)]\{w(i k)\}
$$




\section{Chapter 3}

\section{Flutter Simulation Results}

Simulation results with the time domain, uncorrected frequency domain, and corrected frequency domain methods described in the previous chapter will be presented. The three sets of results will be compared to the benchmark experimental test case available for transonic flutter, the AGARD 445.6 wing. The chapter begins with a description of the experimental setup followed by the time domain, uncorrected frequency domain, and corrected frequency domain results. The time domain and uncorrected frequency domain results obtained by Beaubien are presented below for completeness [1].

\subsection{AGARD 445.6 Test Case}

The AGARD 445.6 experimental test case is one of the three primary transonic flutter experiments. The tests were performed in 1963 at the NASA Langley Transonic Dynamics Tunnel and involved the destruction of a transonic wing geometry under flutter conditions throughout the transonic regime [25]. This test case has since become the benchmark for numerical transonic flutter simulations. The wing model had a NACA $65 \mathrm{~A} 004$ airfoil, an aspect ratio of 4 , a leading edge sweep angle of $45^{\circ}$, a 
taper ratio of 0.66 , and a root chord of $0.58 \mathrm{~m}$. A complete description of the experimental setup can be found in [25]. The laminated mahogany experimental model, seen in Figure 3.1, was weakened with holes drilled through the wing in order to obtain flutter coefficients throughout the Mach number and density ranges of the wind tunnel. As a consequence of using a weakened model, steady and unsteady pressure measurements were unavailable as the wing could not be properly instrumented with pressure taps. The wing was tested throughout the transonic regime and flutter coefficients were determined from Mach 0.338 to Mach 1.141 [25]. The flutter coefficient, a non-dimensional velocity coefficient used to define the flutter boundary, is described as

$$
\bar{U}=\frac{U_{f}}{b_{s} \omega_{\alpha} \sqrt{\frac{m}{V \rho_{f}}}}
$$

where $U_{f}$ is the freestream velocity at flutter, $b_{s}$ is the wing semi-span $(0.2793 \mathrm{~m})$, $\omega_{\alpha}$ is the frequency of the first torsional mode $(39.44 \mathrm{~Hz}), m$ is the wing mass $(1.693$ $\mathrm{kg}), V$ is a volume constant $\left(0.130 \mathrm{~m}^{2}\right)$, and $\rho_{f}$ is the freestream density at flutter.

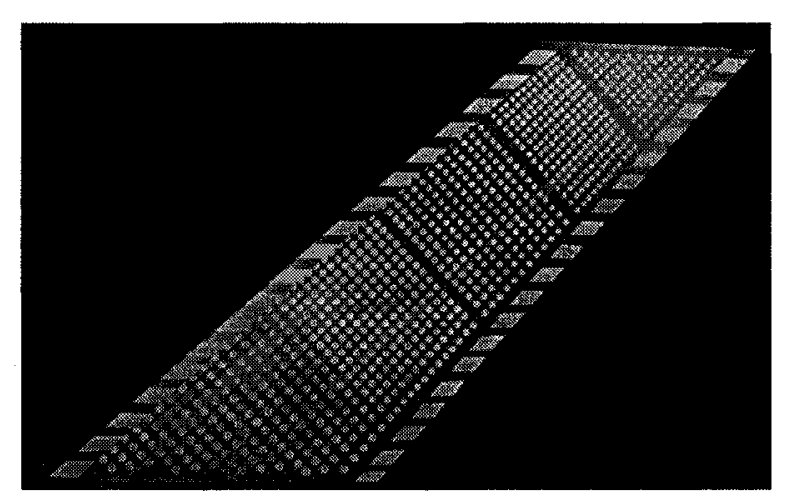

Figure 3.1: The weakened AGARD 445.6 experimental model [25]

\subsubsection{Aerodynamic and Structural Grids}

Three types of grids were required to perform the time and frequency domain simulations, including a time domain grid for the CFD simulations, a DLM aerodynamic 
mesh for the frequency domain solvers, and a structural model for the frequency domain simulations. The mode shapes obtained from the structural model are also input into the integrated beam model for the aeroelastic time domain simulations performed in CMB.

\section{Time Domain CFD Mesh}

Three-dimensional O-O and C-H type grids were created for the Euler and RANS time domain simulations, respectively. The computational domain and cross-section of the O-O grid used for the Euler simulations can be seen in Figure 3.2(a) and a the domain and cross-section of the $\mathrm{C}-\mathrm{H}$ grid used for the RANS simulations can be seen in Figure 3.2(b). The flow in Figures 3.2(a) and 3.2(b) is in the positive $x$ direction and the wing is shaded in black within the computational domain. The cross-sectional views of the two respective grids are shown at the root of the wing. The computational domain in the Euler grid extends 6 root chord lengths in all directions from the wing and the domain in the RANS grid extends 10 root chord lengths from all wing surfaces. The Euler (O-O) and RANS $(\mathrm{C}-\mathrm{H})$ grids contained 27,000 and 45,000 nodes, respectively. A grid convergence study was performed in Reference [1] where it was demonstrated that these mesh densities were sufficient.

Experimental pressures were unavailable for the AGARD 445.6 wing due to the nature of weakening the wind tunnel model, but the accuracy of the computational technique was validated using steady and unsteady simulations performed on the F-5 wing [1], a highly swept wing tested throughout the transonic regime by Tijdeman in 1978 [26]. It was found that similar grid densities to those employed for the AGARD simulations adequately matched experimental results [1]. 

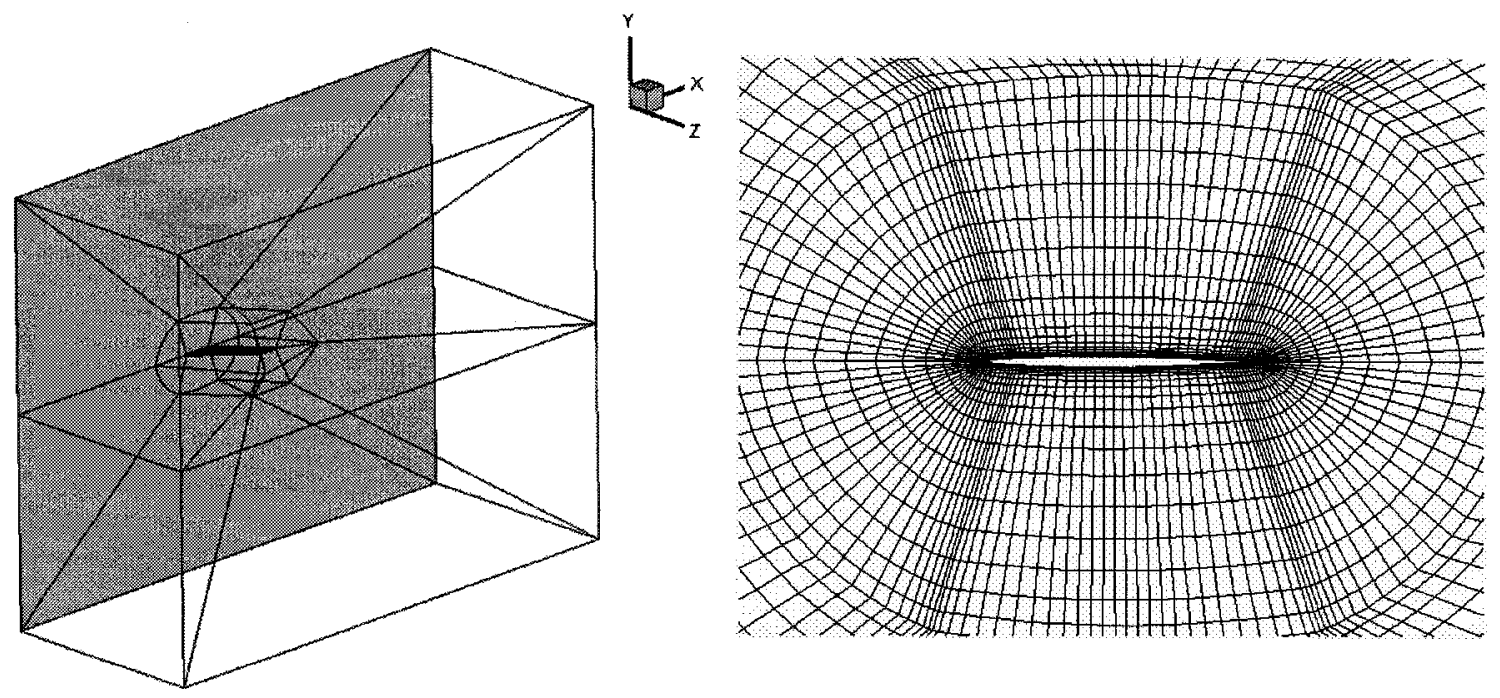

(a) AGARD Euler domain and O-O grid topology
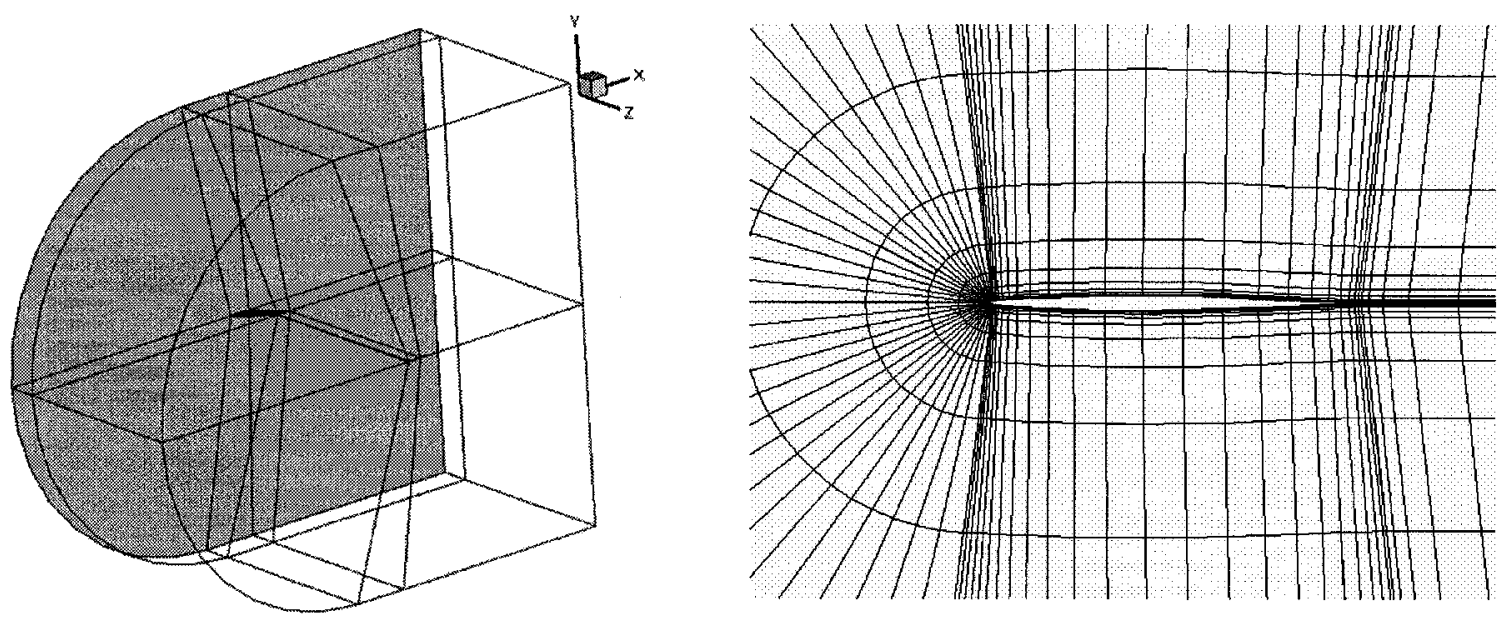

(b) AGARD RANS domain and C-H grid topology

Figure 3.2: Grid topologies used in the Euler and RANS simulations. 


\section{Structural Model}

A structural model of the AGARD 445.6 wing was created for use in the frequency domain flutter analysis using the commercial software MSC/NASTRAN [1]. A 2D orthotropic plate model was developed using the parameters from the aeroelastic optimization study performed by Kolonay [27]. The material properties used were $E_{1}=3.1511 G P a, E_{2}=0.4162 G P a, \nu=0.31, G=0.4392 G P a$ and $\rho=381.98$ $\mathrm{kg} / \mathrm{m}^{3}$, where $E_{1}$ and $E_{2}$ are the moduli of elasticity in the longitudinal and lateral directions, $\nu$ is Poisson's ratio, $G$ is the shear modulus in each plane and $\rho$ is the wing density [27]. The structural model contained 231 nodes and 200 plate elements where the thickness was governed by the airfoil shape. The number of elements compared well with the works of [27] and [19].

The natural frequencies of the first four modes of the modeled wing were obtained using MSC/NASTRAN and are compared to the experimental results obtained for the AGARD 445.6 wing in Table 3.1. The structural parameters from [27] resulted in a wing mass $9 \%$ lighter than the Kolonay model, whose mass was equal to the experimental model [1]. It should be noted that not all wing properties were recorded during the original AGARD experiment, and as such structural models of the AGARD 445.6 wing have one parameter that must be selected in a way that either matches the mass or natural frequencies from the experiment. The simulated modes compared well to the experimental results and so the wing mass in the present work was not adjusted to match the well-known work of Kolonay [27]. Additionally, as structural damping could not be included in the time marching analysis, the structural damping in the MSC/NASTRAN and KEDLMPL models was set to zero as well.

The first four structural mode shapes of the wing determined with MSC/NASTRAN are used in the integrated beam model for the aeroelastic time domain simulations in CMB. 
Table 3.1: Comparison of modal frequencies for the AGARD 445.6 wing.

\begin{tabular}{|c|c|c|}
\hline Mode & $\begin{array}{c}\text { Experiment } \\
{[\mathrm{Hz}]}\end{array}$ & $\begin{array}{c}\text { Beaubien [1] } \\
{[\mathrm{Hz}]}\end{array}$ \\
\hline $1^{\text {st }}$ Bending & 9.60 & 9.46 \\
\hline $1^{\text {st }}$ Torsion & 38.10 & 39.44 \\
\hline $2^{\text {nd }}$ Bending & 50.70 & 49.71 \\
\hline $2^{\text {nd }}$ Torsion & 98.50 & 94.39 \\
\hline
\end{tabular}

\section{Aerodynamic DLM Mesh}

An aerodynamic mesh was required by the DLM solver used in the frequency domain flutter analysis. The linear aerodynamic model contained 400 trapezoidal panels, where the lifting pressures are constant over a given panel. The panels were created using 21 uniformly distributed spanwise and chordwise points to maintain near-unity box ratios, as recommended by [28]. The aerodynamic model does not account for the airfoil thickness.

\subsubsection{Test Conditions}

The flutter boundary of the AGARD 445.6 wing was predicted throughout the range of flow conditions from the transonic flow regime indicated in Table 3.2. Note that for the AGARD experiment modeled here, the location of the flutter boundary is already known and hence these five points will adequately capture the flutter boundary. However, in new wing designs, the location of the flutter boundary is not known $a$ priori and hence a larger number of runs must be performed, emphasizing the need for fast and efficient computational flutter methods.

Also note that the AIC-corrected frequency domain technique could not be applied above a Mach number of 1.0 due to the manner in which the KEDLMPL source code was written. Additionally, an experimental point was not recorded at Mach 0.990 [25], 
Table 3.2: Flow conditions for the AGARD 445.6 flutter tests.

\begin{tabular}{|c|c|c|}
\hline Mach Number & $\begin{array}{c}\text { Density } \\
{\left[\mathrm{kg} / \mathrm{m}^{3}\right]}\end{array}$ & Reynolds number \\
\hline 0.678 & 0.2082 & $1.410 \times 10^{6}$ \\
\hline 0.901 & 0.0995 & $0.911 \times 10^{6}$ \\
\hline 0.960 & 0.0634 & $0.627 \times 10^{6}$ \\
\hline 0.990 & 0.0593 & $0.534 \times 10^{6}$ \\
\hline 1.072 & 0.0552 & $0.442 \times 10^{6}$ \\
\hline
\end{tabular}

but there was interest throughout this work to study the nature of the transonic dip at this Mach number.

\subsection{Time Domain Results}

In order to perform the time domain flutter analysis, the first structural mode of the wing was given an initial upward velocity perturbation and the response was monitored over four complete cycles. If the amplitude response of the modes of the wing result in simple harmonic motion, then the flutter point had been found at a particular Mach number. Simulations were performed iteratively by varying the freestream density between simulations until the flutter point was located. At each time step performed by CMB, the structural loads and aerodynamic loads are computed simultaneously. As such, if the structural model of the wing is modified, then the entire flutter simulation set must be performed again.

The flutter boundary was produced for the aeroelastic RANS and Euler simulations by Beaubien [1], and it can be seen in Figure 3.3 that the boundary was adequately predicted by both flow models, though the RANS simulations more accurately capture the nonlinear transonic effects. It is interesting to note that both flow 
models predicted the same minimum for the transonic dip, although the computationally predicted minimum was different than the experimental minimum indicated near Mach 1.0 in the experimental test report [25]. While an experimental flutter boundary measurement was not taken at Mach 0.990, results from other works near Mach 1.0 indicate that the transonic dip may be more pronounced that the experimental curve suggests $[29,30]$. Additionally, the flutter coefficient at Mach 1.072 was presented in several works to be significantly higher than the experimental data point, as indicated in Table 3.3.

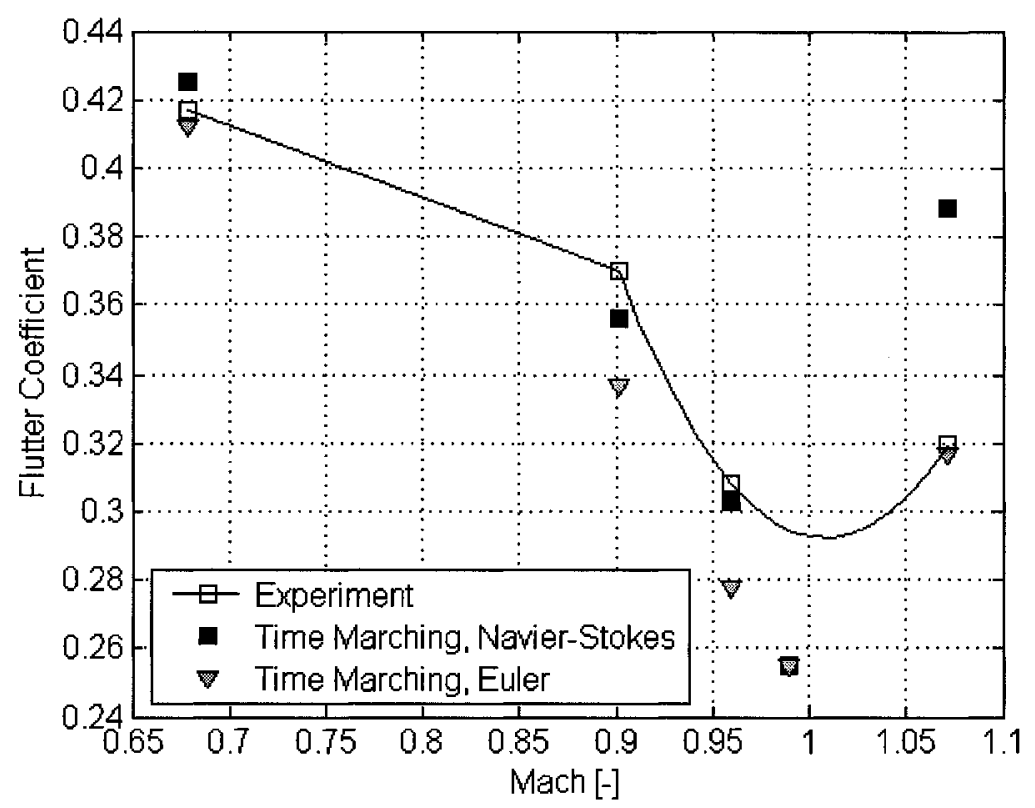

Figure 3.3: Time domain flutter boundary for the AGARD 445.6 wing [1].

It was noted by Beaubien that the difference between the time domain results and the experimental boundary may be attributed to the absence of structural damping in the CFD simulations [1]. Results presented by [32] have suggested that a value of structural damping of $0.5 \%$ will shift the flutter boundary towards the experimental boundary.

While time domain flutter analysis can adequately predict the flutter boundary 
Table 3.3: Flutter coefficient comparison at Mach 1.072.

\begin{tabular}{|c|c|c|}
\hline \multirow{2}{*}{ Reference } & \multicolumn{2}{|c|}{ Flutter Coefficient } \\
\cline { 2 - 3 } & Euler & RANS \\
\hline Goura [12] & 0.452 & - \\
\hline Rausch [10] & 0.466 & - \\
\hline Farhat [31] & - & 0.450 \\
\hline Present Work - CMB [1] & 0.317 & 0.388 \\
\hline Experiment [25] & \multicolumn{2}{|c|}{0.320} \\
\hline
\end{tabular}

for a wing, the large amount of computational time required for the analysis limits the widespread use of time domain flutter analysis in industry. Each RANS flutter simulation required approximately 6 hours and each Euler simulation required approximately 4 hours on a Beowulf cluster of $4 \times 3.2 \mathrm{GHz}$ processors [1]. However, the calculation of the correct flutter boundary point during the design process is an iterative process and may require several simulations. Therefore, while constructing a complete flutter boundary curve for the AGARD test case required $5 \times 6 \mathrm{hrs}=$ $30 \mathrm{hrs}$, this could be as much as 3 to 5 times longer depending on the number of iterations required for a new wing design. Additionally, several other flutter points should be calculated during the design of a wing, limiting the applications of time domain CFD in flutter calculations. As the structural dynamics model is closely coupled to the CFD solver, if the structural model of the wing changes during the design process, the flutter simulation set must be performed again. While the Euler results adequately predicted the flutter boundary of the AGARD 445.6 wing, RANS simulations should be used to accurately capture the nonlinear transonic aerodynamics and can be used to properly predict the flutter boundary of a wing once the design has been frozen. 


\subsection{Uncorrected Frequency Domain Results}

The uncorrected frequency domain flutter boundary results that were obtained using MSC/NASTRAN and KEDLMPL for the AGARD 445.6 wing between Mach 0.678 and Mach 0.990 are depicted in Figure 3.4. The linear frequency domain results were consistently higher than the experimental results and failed to accurately predict the transonic dip that occurs between Mach 0.9 and Mach 1.1. It was noted in [1] that there was a difference between the results obtained with KEDLMPL and MSC/NASTRAN despite the observation that both simulations were performed with the same structural model, DLM aerodynamic model, and used the same KE-flutter solution technique. The source code for MSC/NASTRAN is inaccessible, and so the discrepancy could not be investigated any further [1]

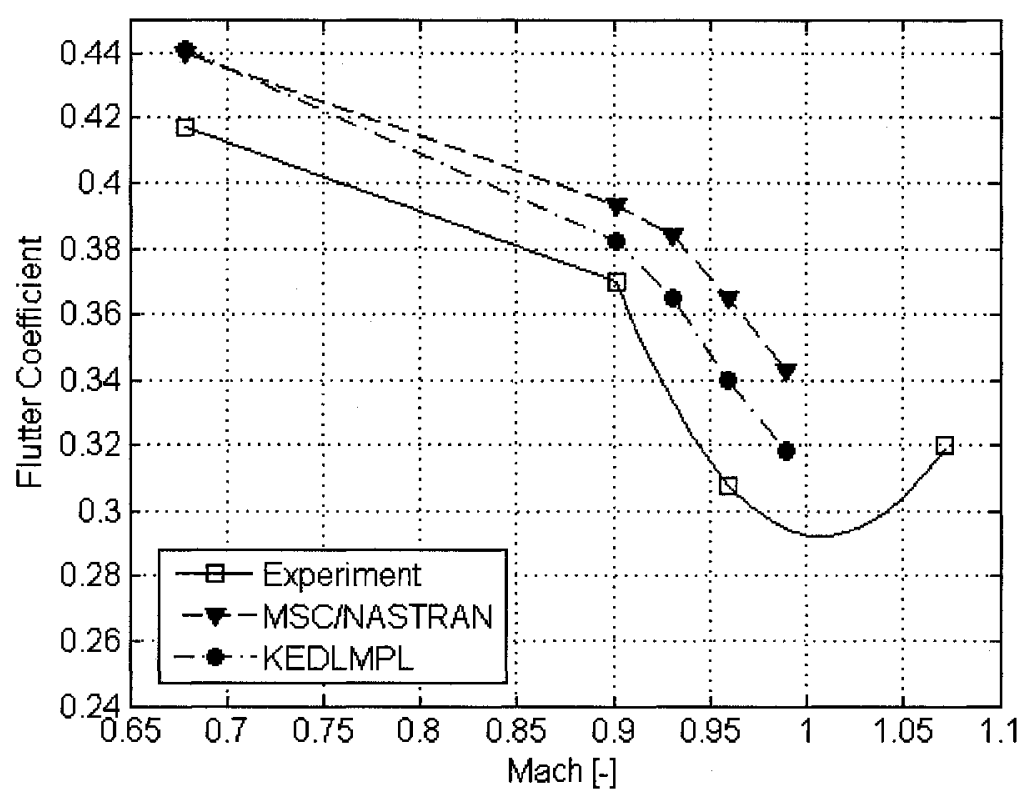

Figure 3.4: Uncorrected frequency domain flutter boundary for the AGARD 445.6 wing [1].

Frequency domain flutter techniques offer significant time savings when compared to time marching methods. Each flutter point calculation required approximately 
5 minutes to compute on a single $2.8 \mathrm{GHz}$ processor with either MSC/NASTRAN or with KEDLMPL. Note that the frequency domain method determines a point on the flutter boundary as a direct result of the simulations. This is in contrast to the time domain method where repeated iterations are required to find the flutter boundary. The current uncorrected frequency domain technique is very attractive for the industry due to the small amount of time involved in the calculation of the flutter boundary despite the large inaccuracies found in the transonic regime. A second advantage of frequency domain methods is that if the internal structure of the wing is changed, only the structural model needs to be modified whereas the aerodynamic model is unchanged, making this method particularly useful during the design process.

\subsection{Corrected Frequency Domain Results}

The AIC-correction procedure was implemented in KEDLMPL and used nonlinear lifting pressures from rigid-body harmonic oscillation simulations performed using the RANS equations in CMB. Based on the work of [19], the pitching oscillations were run for two cycles, with a resolution of 20 steps per cycle, and pressures were obtained from the second cycle to eliminate the effect of initial transients. The reduced frequency selected for the oscillatory simulation at a particular Mach number can correspond to the flutter frequency predicted by the linear frequency domain solver. Alternatively, the experimental flutter frequency at a particular Mach number can also be used for the reduced frequency of the oscillatory CFD simulations [19].

The real and imaginary lifting pressures obtained with the DFT described in Equations 2.28 and 2.29 were verified using CFD results produced by Silva [19] as experimental pressures do not exist for the AGARD 445.6 wing. The real and imaginary lifting pressures at $30.8 \%$ span are compared at four Mach numbers in Figure 3.5. 
Simulations were not performed by Silva at Mach 0.990 , but the unsteady pressures obtained with CMB are shown in Figure 3.5(d). Real and imaginary pressures were only presented by Silva at $30.8 \%$ span and were the only unsteady pressure results available for comparison for the AGARD wing. The differences observed between the real and imaginary pressures in Figure 3.5 may be attributed to the different computational schemes used for the comparison. The work of Silva [19] used a finite difference solution of the Navier-Stokes equations with an algebraic Baldwin-Lomax turbulence model, whereas CMB used in this work used a finite volume solution of the Navier-Stokes equations with a shear stress transport turbulence model [1]. The CFD grid used by Silva contained 145,000 nodes with 120 points located in the chordwise direction on the lifting surface, whereas the present work used a grid of 45,000 nodes with 20 points located in the chordwise direction along the lifting surface. As such, the prediction of the movement of the shock during the unsteady pitching simulations performed in CMB may have differed from the predictions of Silva [19].

As a secondary verification of the unsteady nonlinear pressures, the amplitude and phase from the transformed lifting pressures were compared to results from [19] at $30.8 \%$ span at Mach 0.960 as seen in Figure 3.6.

The flutter velocity was obtained from the velocity-damping diagrams output from the corrected frequency domain simulations. The corrected frequency domain velocity-frequency and velocity-damping diagrams at the four simulated Mach numbers can be seen in Figures 3.7 and 3.8. The flutter coefficient at each Mach number was then determined using Equation 3.1.

The flutter boundary computed with the AIC-correction technique is compared to experimental results and the computational results of Reference [19] in Figure 3.9 and agrees well with the experiment. A comparison with results from the RANS solution in Table 3.4 indicates that the transonic dip minimum is well characterized by the AIC-corrected solver. 

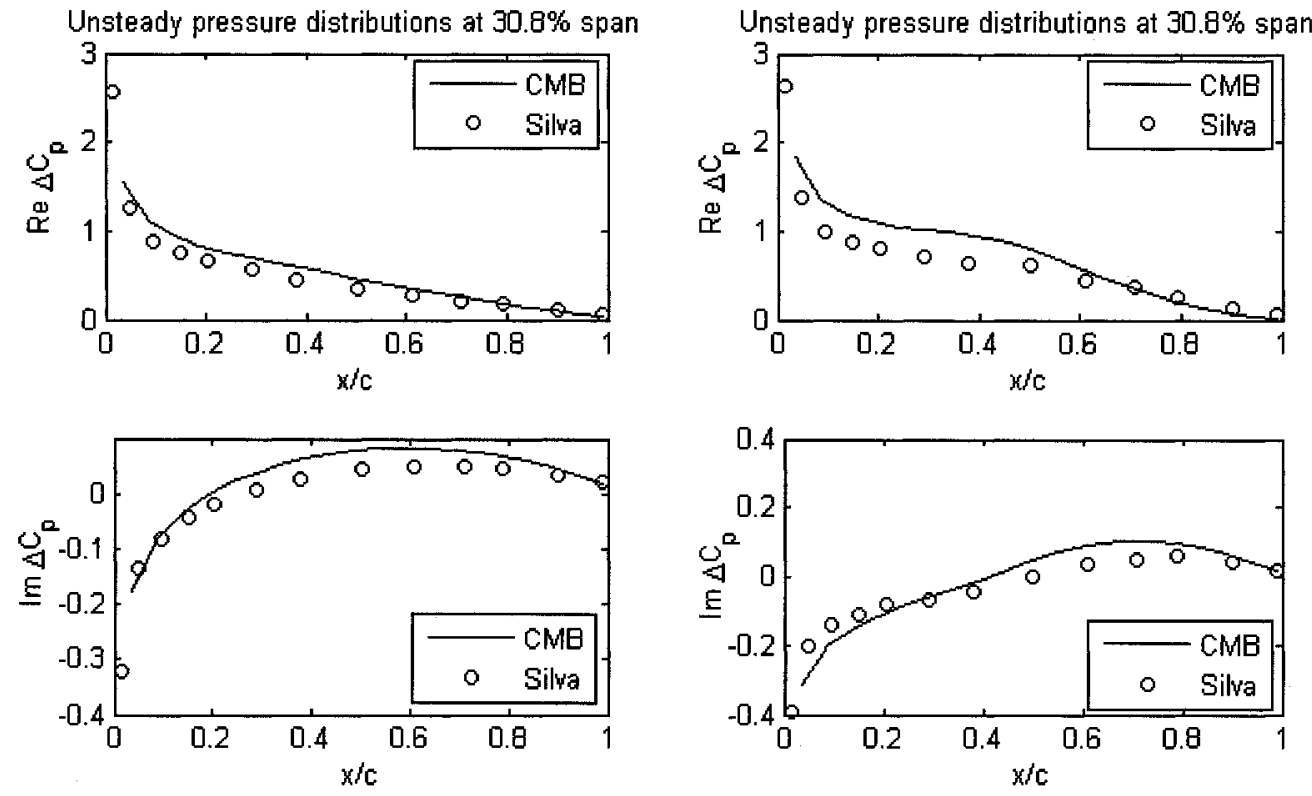

(a) Mach 0.678

(b) Mach 0.901
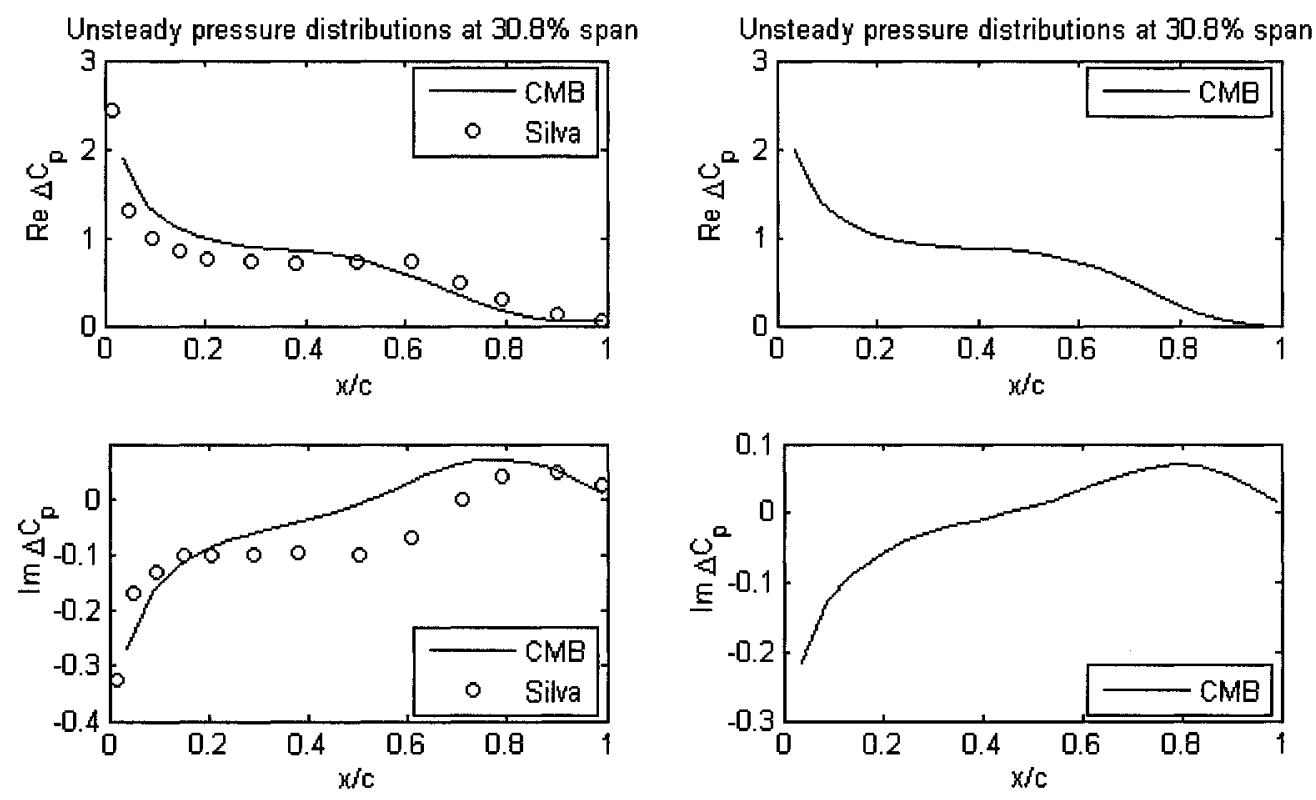

(c) Mach 0.960

(d) Mach 0.990

Figure 3.5: Real and imaginary unsteady pressures for the AGARD 445.6 wing 

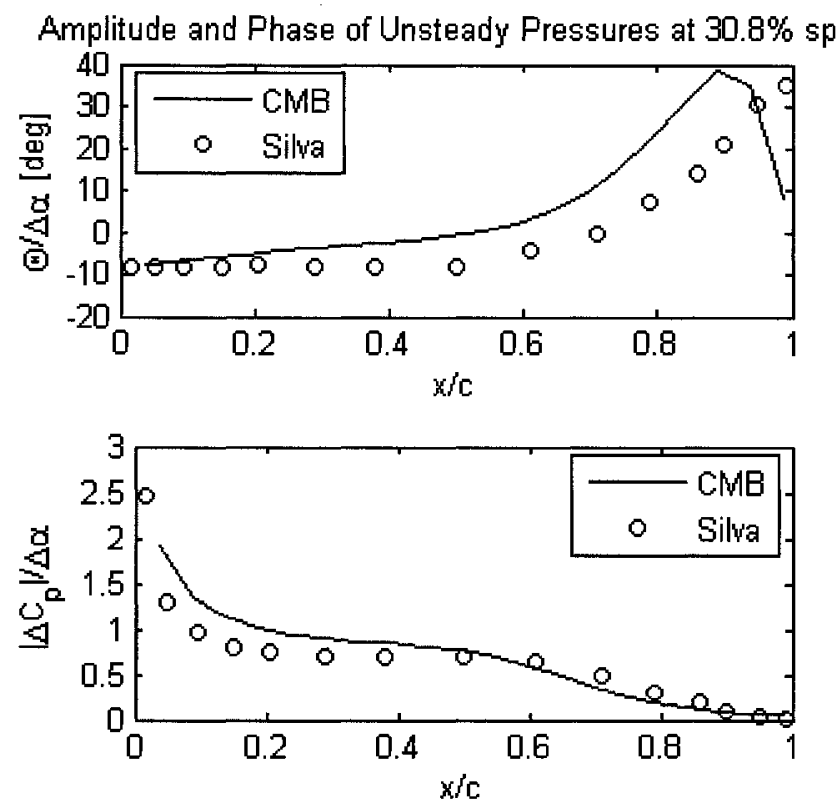

Figure 3.6: Amplitude and phase of the unsteady pressures at Mach 0.960

Table 3.4: Flutter coefficient comparison at Mach 0.99 .

\begin{tabular}{|c|c|c|}
\hline Reference & Method & Flutter Coefficient \\
\hline Lee-Rausch [11] & Euler & 0.310 \\
\hline AIC-Corrected & Frequency & 0.281 \\
\hline$C M B$ & RANS & 0.255 \\
\cline { 2 - 3 } & Euler & 0.255 \\
\hline Experiment [25] & Wind Tunnel & N/A \\
\hline
\end{tabular}

Each flutter point predicted with the AIC-correction technique required approximately three hours to compute, a significant decrease in simulation time compared to RANS simulations. The oscillatory pitch simulations took approximately two to three hours to compute on a single $2.8 \mathrm{GHz}$ processor, post processing required five to ten minutes in MATLAB, and the flutter simulation required approximately five minutes using KEDLMPL. Therefore, the total time of obtaining one point on the flutter boundary line is between 3 and 4 hours. Although the corrected frequency domain technique requires significantly more time than the uncorrected frequency 

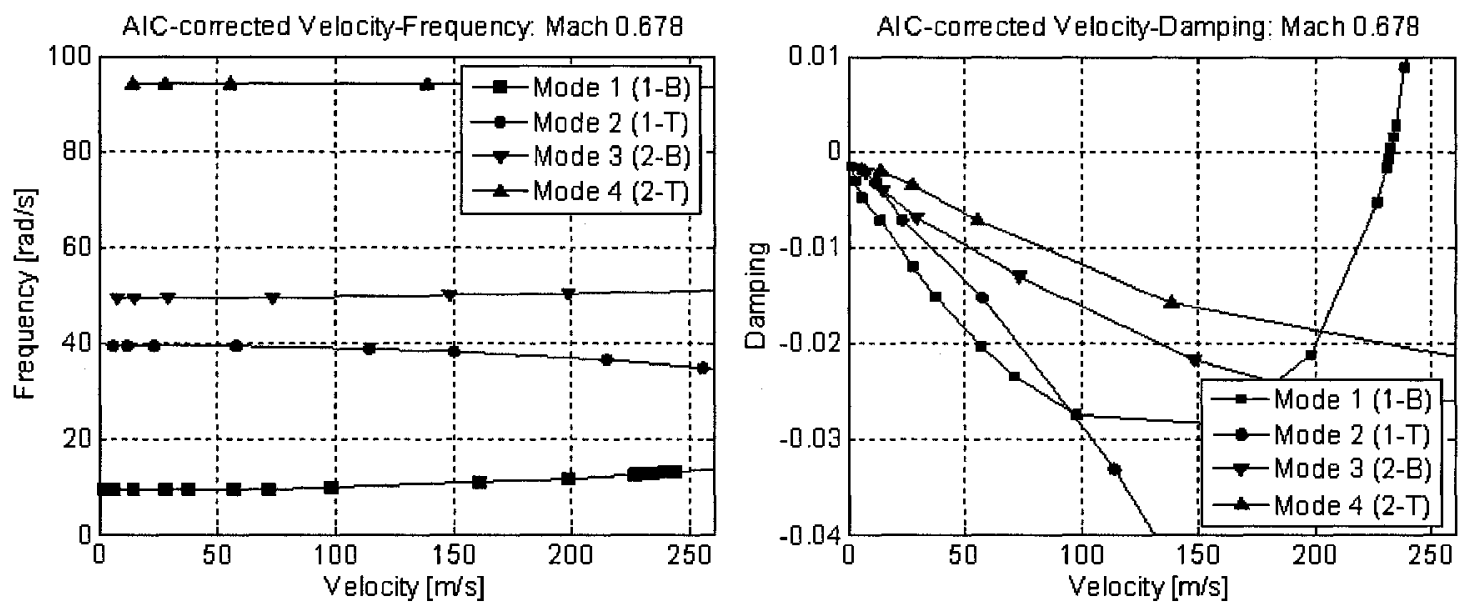

(a) Mach 0.678
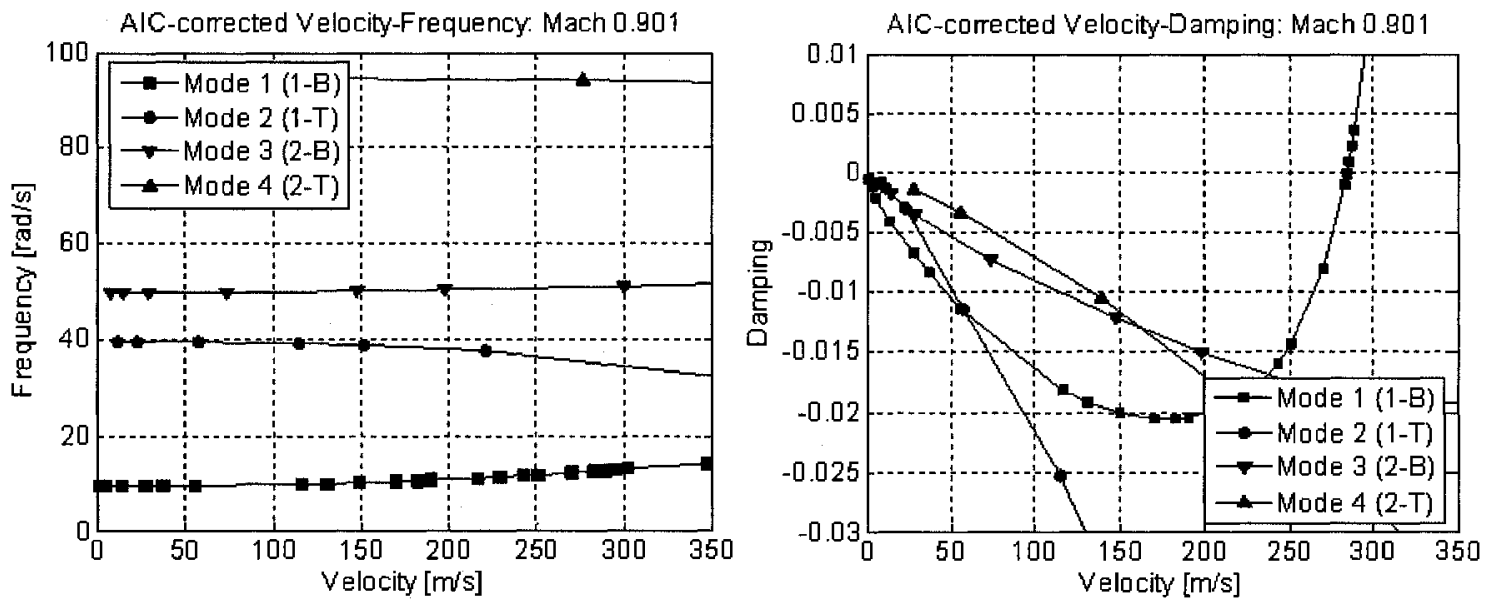

(b) Mach 0.901

Figure 3.7: AIC-corrected velocity-frequency and velocity-damping plots at Mach 0.678 and Mach 0.901 . 

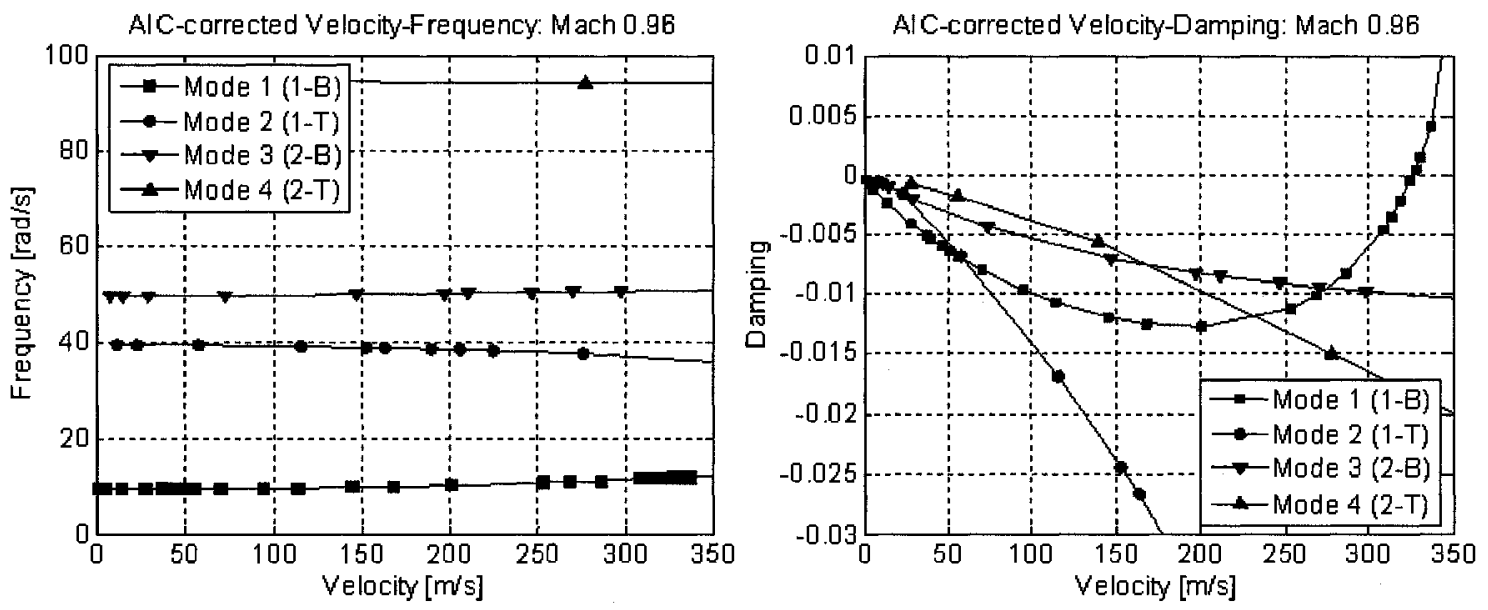

(a) Mach 0.960
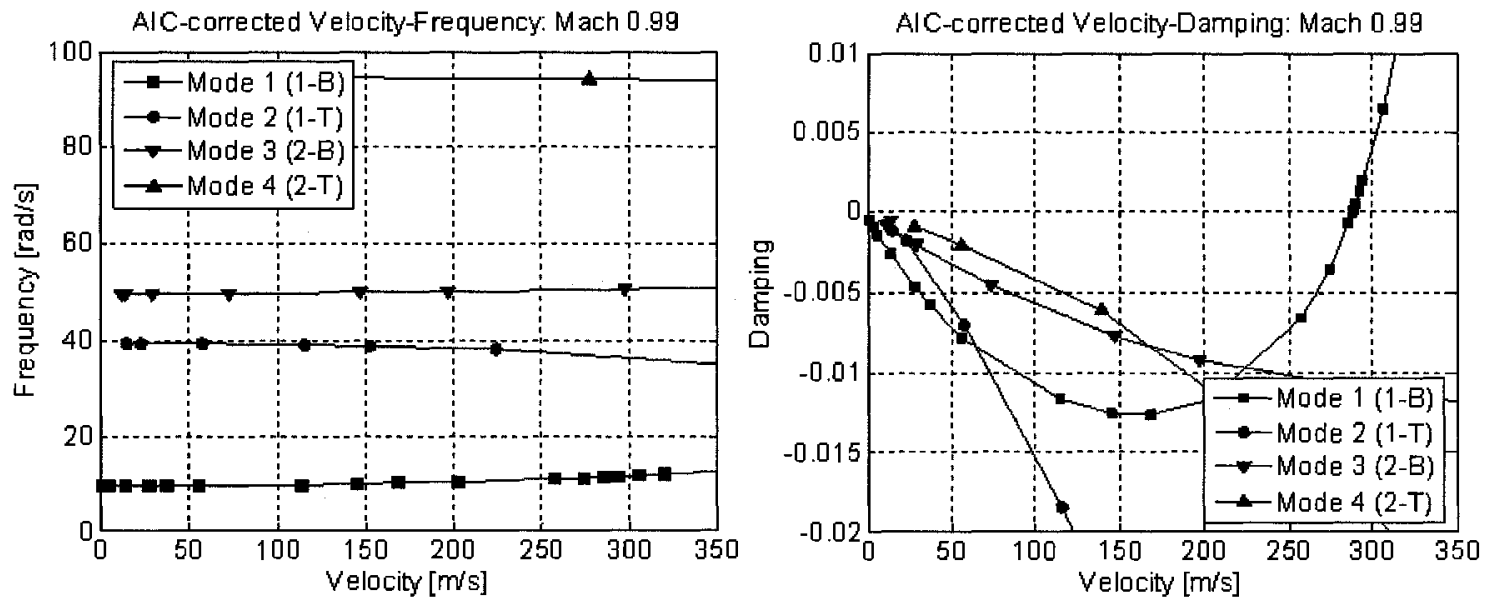

(b) Mach 0.990

Figure 3.8: AIC-corrected velocity-frequency and velocity-damping plots at Mach 0.660 and Mach 0.990 . 


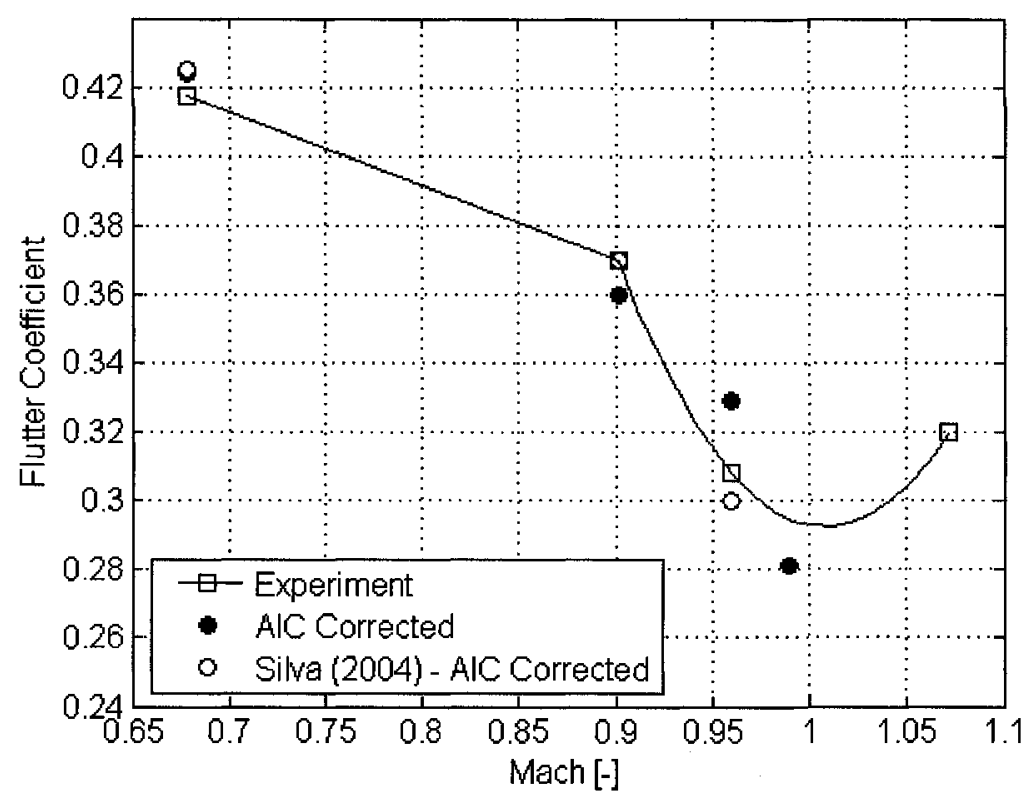

Figure 3.9: Corrected flutter boundary for the AGARD 445.6 wing.

domain solvers ( 5 minutes per flutter point), the correction method presents a substantial time savings compared to time domain CFD flutter analysis (up to 30 hours per flutter point) while accounting for the same nonlinear aerodynamics. The corrected frequency domain method is approximately 7 to 8 times faster for a single simulation than the time marching aeroelastic analysis, as repeated time domain simulations are required to find a single flutter point. As such, a flutter point can be determined in a matter of hours instead of days of computational time. Additionally, unlike a closely-coupled CFD solver, the AIC-correction technique corrects only the aerodynamic matrices involved in the DLM solver, and changes can be made to the structural model without affecting the nonlinear pressures involved in the correction. 


\subsection{Conclusion}

Computational transonic flutter analysis was performed on the AGARD 445.6 wing in the time domain and in the frequency domain. A procedure was developed to correct the frequency domain flutter boundary by accounting for the nonlinear aerodynamics present within the transonic regime using rigid body unsteady pitching-motion CFD simulations. Time domain aeroelastic CFD simulations and AIC-corrected frequency domain results both adequately predicted the flutter boundary of the AGARD 445.6 wing. The corrected frequency domain approach however, required significantly less computational time and fewer computational resources than the time domain CFD analysis, rendering the method attractive for industrial use during the design phase of a wing. The corrected frequency domain approach is ideal for iterative design processes since the oscillatory pitch aerodynamic simulation only needs to be performed once per Mach number and is not affected by the structural model. As such, the effect of structural changes on the flutter boundary can be assessed very rapidly during the design process.

\subsection{Recommendations and Future Work}

As the RANS time domain and AIC-corrected frequency domain solutions both have useful industrial applications, further validation should be conducted on another transonic wing model that more closely resembles a commercial transport wing. Potential candidates could include the YXX wing, the PAPA wing, or the LANN wing. The LANN wing experiment was performed in the 1980s and uses a supercritical airfoil but was never tested to flutter because of the operational limits of the wind tunnel. Aerodynamic and structural information is available however, and RANS simulations could be used as the benchmark comparison to the AIC-corrected results. 
The capability of CMB and the AIC-correction method to perform time and frequency domain flutter analysis of a wing with a pod-mounted engine should be investigated. The simulation of a wing with an engine would further increase the industrial applications of the AIC-corrected flutter technique. 


\section{Part II}

\section{Rotary-wing Aeroelasticity}




\section{Chapter 4}

\section{Introduction to Wind Turbine Technology}

In the second part of this thesis, the aeroelastic simulations of a wind turbine modeled with a vortex particle wake and a nonlinear structural model will be presented. The focus was on identifying and developing a computational tool feasible for practical industrial applications that is both computationally robust and efficient. The chapter will start with a historical review of wind turbine development, followed by a review of the computational tools currently used for predicting their performance. This will help to identify the need for more accurate aerodynamic and aeroelastic simulations. Aeroelastic simulations of a benchmark experimental wind turbine test are then presented.

\subsection{Historical Developments}

Wind energy is rapidly becoming a viable source of renewable energy and worldwide installed wind energy capacity is growing by $25 \%$ annually [33]. The modern threebladed horizontal axis wind turbine is now used exclusively for electrical generation, and represents the culmination of thousands of years of design and development of ancient windmills.

Early windmills were typically used for milling grain or pumping water and may 
have been invented between 2,000 and 3,000 years ago in Egypt, though the existence of these early windmills is disputed in literature $[34,35]$. The first formally documented windmills appeared in Persia and Afghanistan between the 7th and 12th centuries. These early windmills were vertical axis windmills used for milling grains, as seen in Figure 4.1(a) [34]. The European horizontal axis windmill was developed independently in northern Europe between the 12th and 18th centuries. These were traditionally drag-driven windmills with four wooden or cloth covered sails that were used for pumping water, for milling grain, or as sawmills, as depicted in Figure 4.1(b). Continuous improvements and refinements were made to the towers, sails and control devices of European windmills between the 12th and 17th centuries, but these were conducted on a trial and error basis [35]. It wasn't until the 17th and 18th centuries that windmill design and aerodynamics were influenced by and studied by Leibniz, Bernoulli, and Euler [34]. A third distinct type of windmill was developed in the United States by Daniel Halladay in the late 19th century, an example of which can be seen in Figure 4.1(c). The American wind turbine, which was primarily used for irrigation and pumping, was the first successful commercial windmill and had sold over six million examples by 1930 [34].

The development of wind turbines for electrical generation began in the late 19th century with the construction of the Brush windmill in Ohio. The Brush turbine had 144 thin blades, a rotor diameter of $17 \mathrm{~m}$, and supplied $12 \mathrm{~kW}$ of DC power for charging batteries [35]. This turbine successfully generated power for twenty years before being decommissioned and was the last example of a large multi-vane wind turbine. Shortly after the Brush windmill was completed, the La Cour windmill was built in Denmark in 1891 and improved upon many of the technical achievements of the European windmills. The La Cour windmill had four twisted and cambered blades that had been tested and developed in a wind tunnel. Successive industrialized iterations of this design produced between 10 and $35 \mathrm{~kW}$ of electricity [34]. 
The precursor to the modern wind turbine however, was the Smith-Putnam turbine developed in 1941 in Vermont, whose rotor blades were designed in part by von Kármán. The Smith-Putnam turbine was the first megawatt scale turbine, as seen in Figure 4.1(d), and produced up to 1.25 MW of power. The turbine had a two-bladed stainless steel rotor with a diameter of $53 \mathrm{~m}$ and featured many novel innovations including full-span pitch control, flapping hinges to alleviate load imbalances, and active yaw control. The Smith-Putnam turbine fed electricity into the local grid for four years before developing large cracks in its rotor. The turbine had to be dismantled as there were no funds to repair its rotor, but it pioneered most of the technologies that have been implemented in modern turbines [34].

Interest in wind turbines diminished following the Second World War because of cheap oil imports and interest in nuclear energy [36]. However, the United States launched an ambitious research and development program for new turbine designs after the energy crisis in the 1970s. New experimental wind turbines were tested and a large number of wind farms were built in the United States throughout the mid 1980s [35]. The vast majority of new wind farm developments since the 1980s have been built in Europe as interest again faded in North America due to inexpensive oil in the 1990s and Europe now accounts for $60 \%$ of the worldwide wind generating capacity [33].

There has been a push to increase the rotor size of commercial turbines throughout the past twenty years due to the economy of scale, increased power generation requirements, and more effective land use. The increase in turbine size and power since 1980 is represented schematically in Figure 4.2. Though current designs exist for $5 \mathrm{MW}$ turbines, the typical size of new turbines in wind farms is between 2 and $3 \mathrm{MW}$ with rotor diameters of $80 \mathrm{~m}$ to $100 \mathrm{~m}$.

Wind turbine research in Canada reached its peak in the 1970s and 1980s and focused on the development of Darrieus vertical-axis wind turbines [37]. The research 


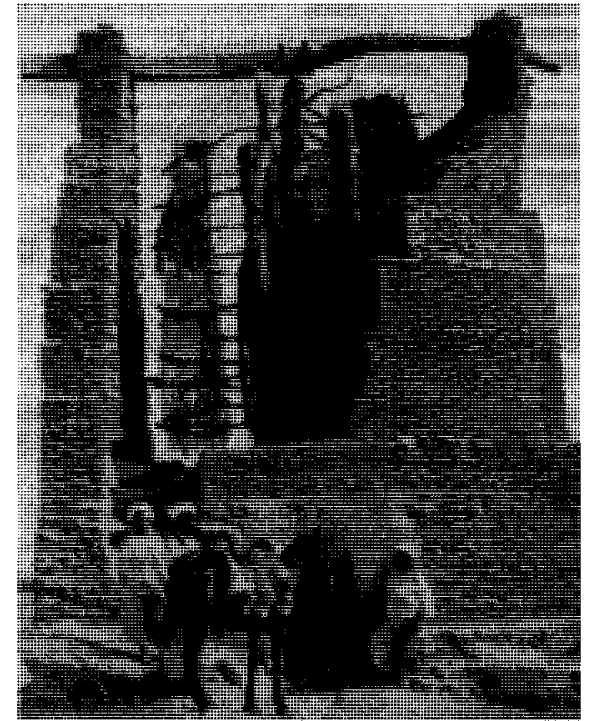

(a) Vertical axis windmill in (b) Afghanistan [34]

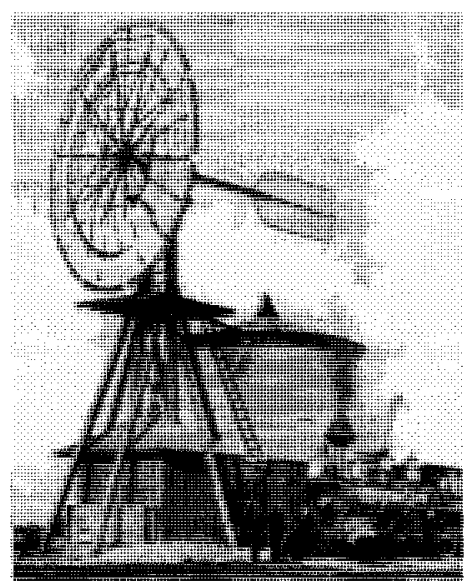

(c) American water pumping (d) windmill [34]

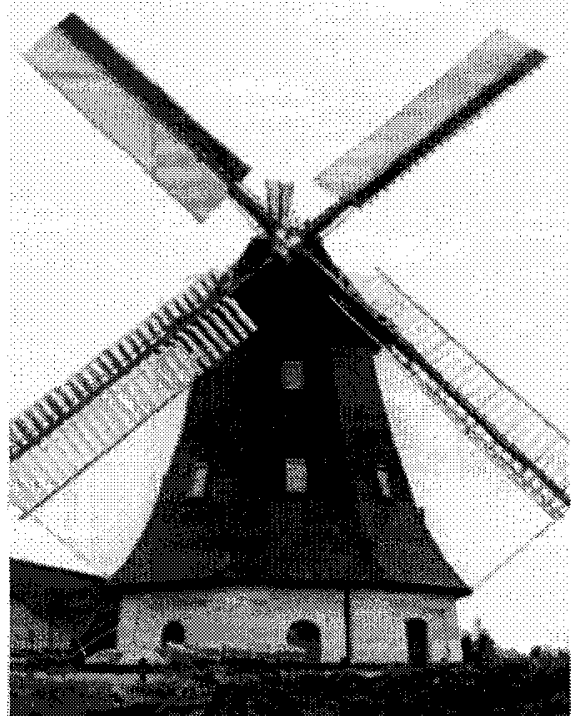

(b) Dutch horizontal axis windmill [34]

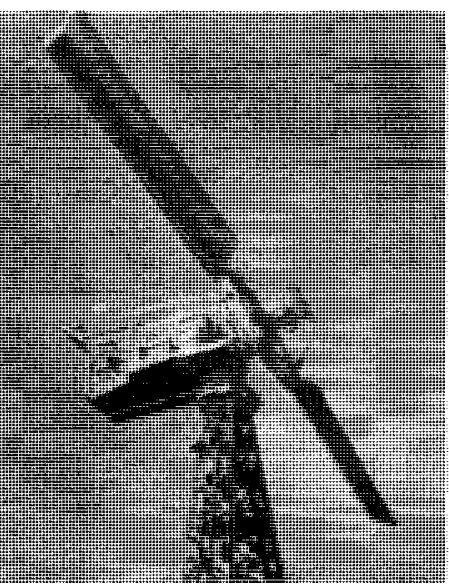

(d) Smith-Putnam wind turbine [34]

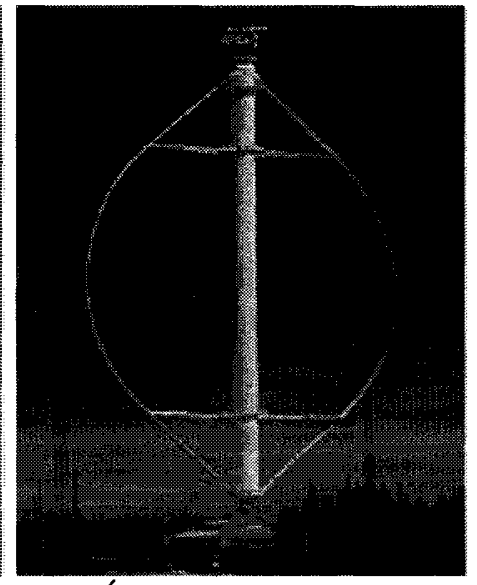

(e) Éole vertical axis wind turbine (www.windworks.org).

Figure 4.1: The development of wind turbines. 


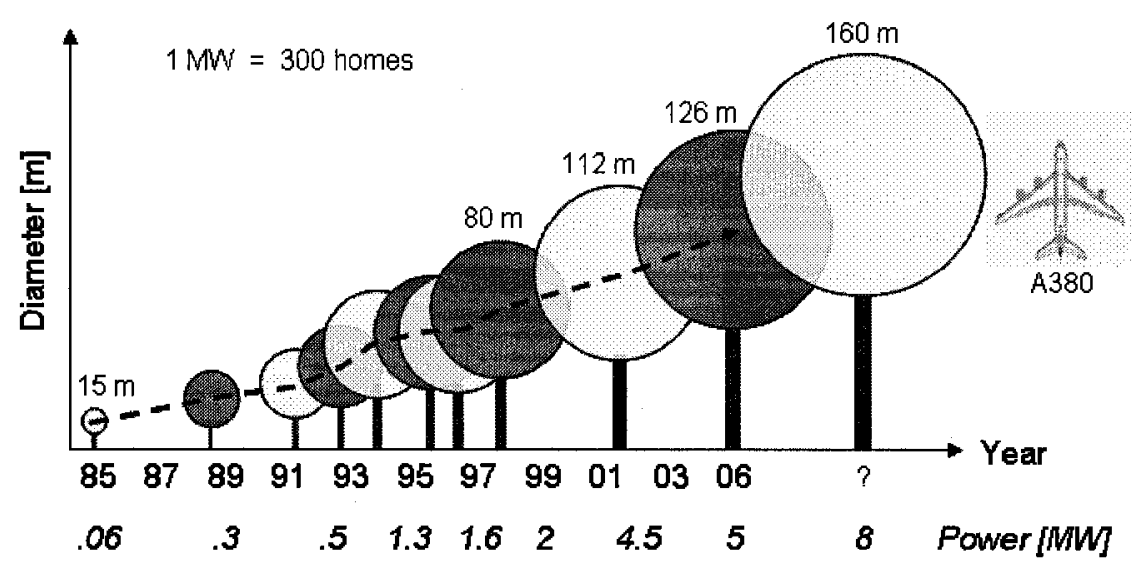

Figure 4.2: Growth of modern horizontal axis wind turbines.

Adapted from www.renewableenergyworld.com.

carried out by the National Research Council of Canada led to the construction of the 4 MW Éole vertical-axis turbine in Quebec in 1987, the first Megawatt-scale vertical axis wind turbine, shown in Figure 4.1(e). Virtually all wind turbines in Canada however are three-bladed horizontal axis turbines and there has been little development of Darrieus-style turbines since the Éole project. The first commercial wind farm in Canada was created in southern Alberta in 1993, and installed capacity in Canada has only recently demonstrated significant growth [38]. The total installed wind energy capacity in Canada increased from $19 \mathrm{MW}$ in 1994 to $444 \mathrm{MW}$ in 2004 and then to $1850 \mathrm{MW}$ in 2008, and several large-scale projects have been planned for the next decade $[39,38]$. Worldwide installed wind generating capacity reached $94 \mathrm{GW}$ in 2007 and has demonstrated sustained annual growth of $25 \%$ as the cost of wind energy decreases and the need for renewable energy technologies increases [33].

\subsection{Anatomy of a Horizontal Axis Wind Turbine}

Wind turbine designs can be classified as either vertical axis wind turbines or horizontal axis wind turbines (HAWT). The primary components of a HAWT can be seen in Figure 4.3. A small amount of research continues on vertical axis turbines, 
but over $95 \%$ of currently-installed commercial turbines are HAWTs [36]. A large amount of research was performed in the United States of America in the 1980s on large two-bladed wind turbines, but the majority of new designs use three blades. Two-bladed rotors require teetered hubs in order to alleviate asymmetric loading and as a result, three-bladed turbines with rigid hubs have become the industry-standard.

Though wood and metal were typically used for older turbine blades, new designs are primarily composed of glass or carbon fibre reinforced polymers. A thorough review of rotor blade materials and construction techniques is given in [34]. Most new large turbines include a blade pitch mechanism in the hub, which is designed to adjust the blade pitch angle to control the power output of a turbine and to aerodynamically brake the rotor by rotating the blades $90^{\circ}$ into the feathered position [34]. The rest of the mechanical drive train includes the low-speed shaft, bearings, a speed-up gearbox, and a generator. Wind vanes and cup anemometers are installed on top of the nacelle downwind of the rotor and estimate the prevailing wind speed and direction. Based on these measurements, control systems modify the blade pitch angle to control power output and modify the yaw angle of the turbine to obtain normal flow through the rotor. The yaw system automatically orients the rotor and nacelle into the wind in order to reduce the instances of yawed flow, which cause asymmetric loading on the rotor blades and reduced power generation.

\subsection{Power Generation}

The power generated by HAWTs can either be regulated using pitch-controlled or stall-controlled turbine blades.

In pitch-controlled units, the pitch angle of the turbine blades is adjusted based on the incident wind speed measurements during operation to maintain constant rotor rpm and to regulate power output. This allows the angle of attack to remain low 


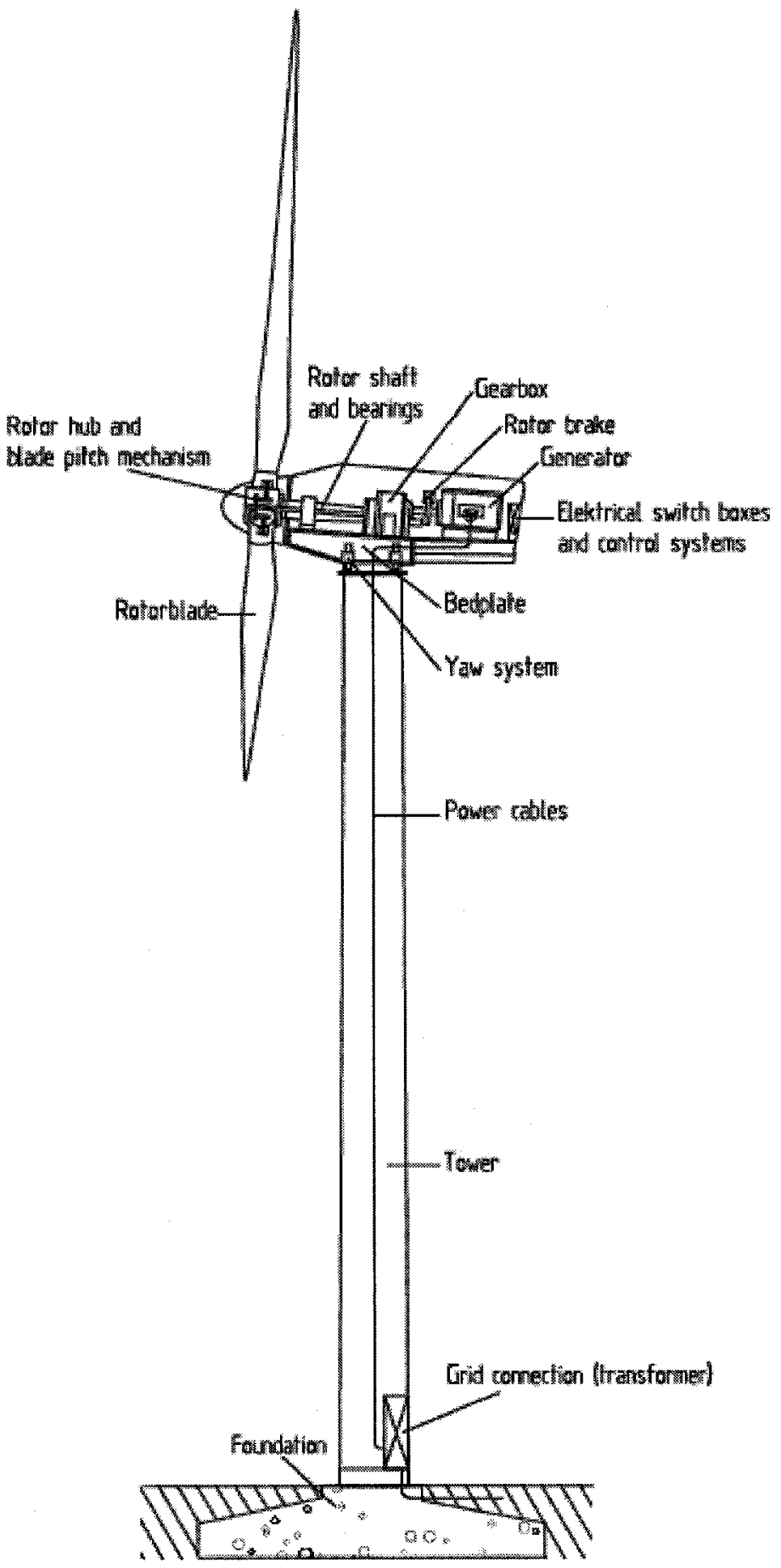

Figure 4.3: Primary components of a HAWT. Reproduced from [34]. 
throughout the operational regime of the turbine and allows the rated turbine power to be strictly controlled over the rated wind speeds. If the incident velocity is too high and risks damaging a machine, the blades are rotated to the feathered position and the turbine is stopped [34].

Stall-controlled turbines however, have a fixed blade pitch angle and are designed to operate in the fully stalled flow regime over the range of rated wind speeds. Stallregulated blades rely on passive stall control to regulate the power generated by the rotor and to maintain constant rotor rpm. This phenomena is described in Figure 4.4 where a turbine blade section is shown at both a low wind speed and a high wind speed. The fixed blade pitch angle $(\theta)$ is assumed to be positive towards the feathered position, and the tangential velocity $\left(V_{\text {rot }}\right)$ of a particular spanwise section is assumed to be constant throughout the operational range of the turbine. Figure 4.4 demonstrates that the blade section angle of attack $(\alpha)$ will increase with the wind speed. In wind speeds that correspond to the rated power of a turbine, $\alpha$ will be higher than the critical angle of attack $\left(\alpha_{C R I T}\right)$, where the blade section begins to experience stalled flow. At the rated turbine power, a varying degree of stall occurs over the blade sections and the rotor power is controlled by the amount of aerodynamic stall occurring over the span of the rotor blade. Stall-regulated rotors are typically only used on smaller wind turbines and suffer from restrictions for grid connection.

Wind turbine performance is described by a power curve, which identifies the cutin velocity, the rated power, the rated wind velocity, and the cut-out velocity. The cut-in velocity refers to the velocity where the turbine can start to deliver power and typically occurs around 3 to $4 \mathrm{~m} / \mathrm{s}$. The rated wind velocity is the wind speed at which the rated generator power is reached, and the cut-out velocity is the highest wind velocity at which the turbine can be operated without damage [34]. If the wind velocity exceeds the cut-out velocity, the turbine is braked aerodynamically or 

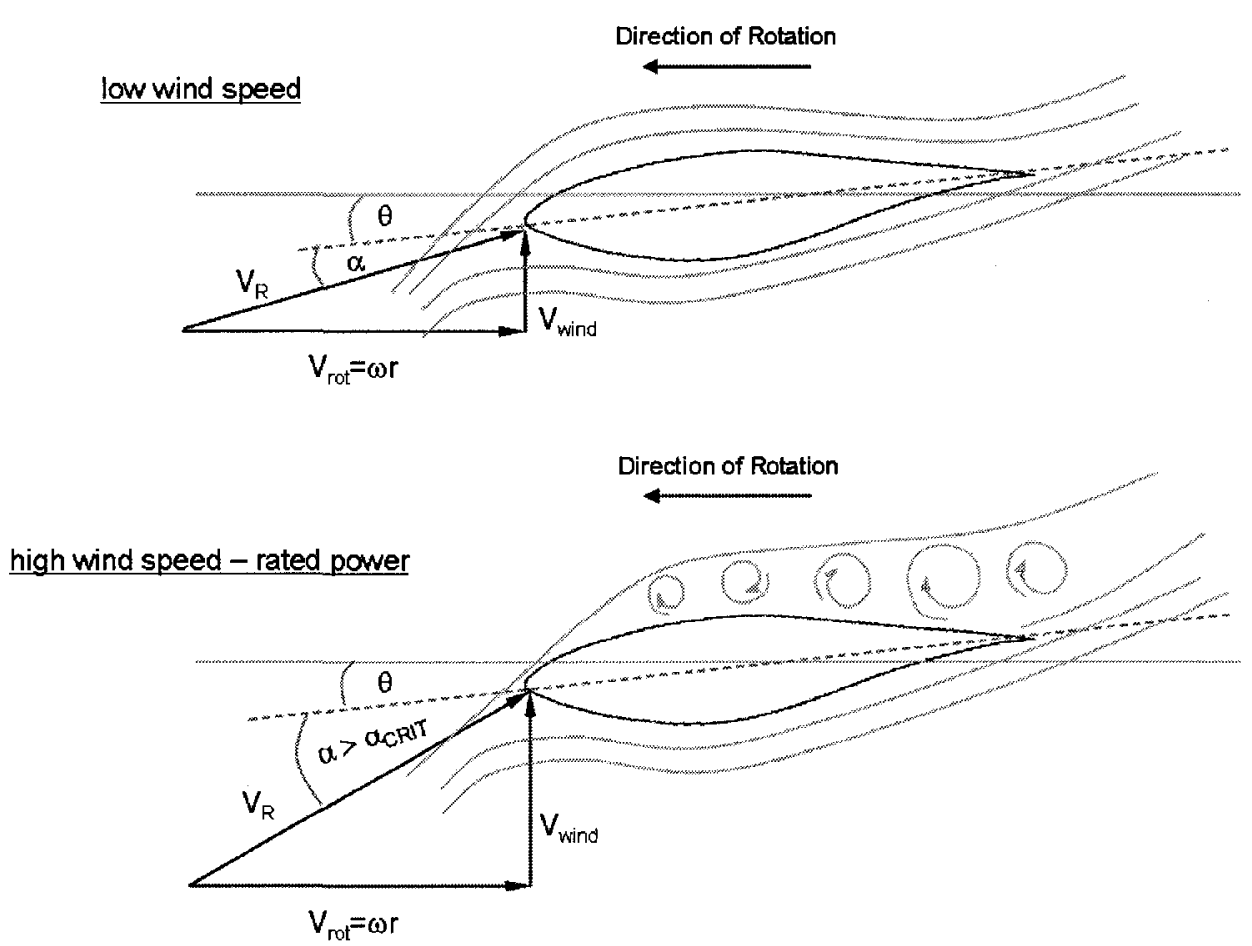

Figure 4.4: Process of stall control.

mechanically and will no longer generate power. A schematic of a typical power curve is shown in Figure 4.5 and includes the difference in power output at the rated power level between stall-controlled and pitch-controlled turbines. The rated power usually occurs at wind speeds higher than $12 \mathrm{~m} / \mathrm{s}$ and the cut-out speed is typically $25 \mathrm{~m} / \mathrm{s}$. At wind speeds below the rated speed however, there will be a high degree of sensitivity in generated power.

The American Wind Energy Association has developed a classification system for the annual mean wind speed at 50 metres above the ground, as seen in Table 4.1 [40]. Ideal wind farm sites have annual mean wind speeds greater than $8.8 \mathrm{~m} / \mathrm{s}$ with a wind power density of at least $800 \mathrm{~W} / \mathrm{m}^{2}$. Wind turbines operate predominantly in wind speeds of approximately 7 to $8 \mathrm{~m} / \mathrm{s}$, where the turbine power curve (Figure 4.5 ) has high sensitivity, despite the fact that commercial wind turbines reach their rated power at approximately $12 \mathrm{~m} / \mathrm{s}$. 


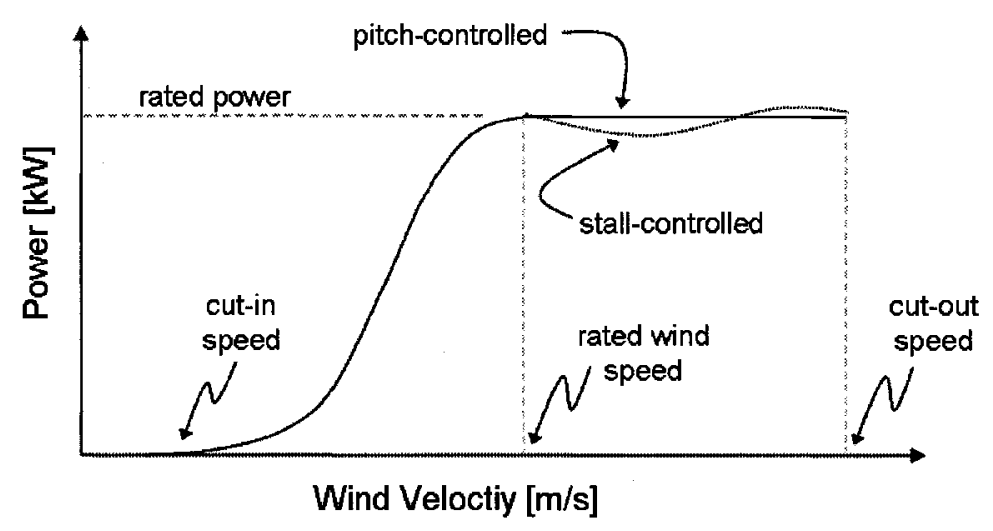

Figure 4.5: Wind turbine power curve.

Table 4.1: American Wind Energy Association wind classes at a height of $50 \mathrm{~m}$ [40].

\begin{tabular}{|c|c|c|}
\hline Class & $\begin{array}{c}\text { Wind Speed } \\
{[\mathrm{m} / \mathrm{s}]}\end{array}$ & $\begin{array}{c}\text { Power Density } \\
{\left[\mathrm{W} / \mathrm{m}^{2}\right]}\end{array}$ \\
\hline 1 & $<5-6$ & $<200$ \\
\hline 2 & $5.6-6.4$ & $200-300$ \\
\hline 3 & $6.5-7.0$ & $300-400$ \\
\hline 4 & $7.0-7.5$ & $400-500$ \\
\hline 5 & $7.5-8.0$ & $500-600$ \\
\hline 6 & $8.0-8.8$ & $600-800$ \\
\hline 7 & $>8.8$ & $>800$ \\
\hline
\end{tabular}

\subsection{Aerodynamic and Structural Loading}

The aerodynamic environment of wind turbines, similar to helicopters, involves significant unsteady three-dimensional effects. Their operational environment includes the effects of atmospheric turbulence, the atmospheric boundary layer, directional and temporal variations in wind shear and thermal stratifications [41]. These unsteady effects cause a wide range of unsteady loads, which are compounded by the effects of turbine yaw misalignment, tower and hub interference, wake dynamics, and turbine wake interaction in wind farms. These effects can be observed by comparing the wake 
behind an NREL turbine in an outdoor environment as seen in Figure 4.6(a) to the wake behaviour of the same turbine operating in the NASA Ames wind tunnel in Figure 4.6(b). As the unsteady loading environment of operational turbines makes it difficult to validate computational tools, a limited number of wind tunnel experiments have been performed and can be used for code validation [42]. A schematic of the loads and stresses acting on a turbine rotor can be seen in Figure 4.7. The thrust and torque output of a wind turbine determine its performance as they relate to the velocity deficit in the wake and the turbine output power, respectively.

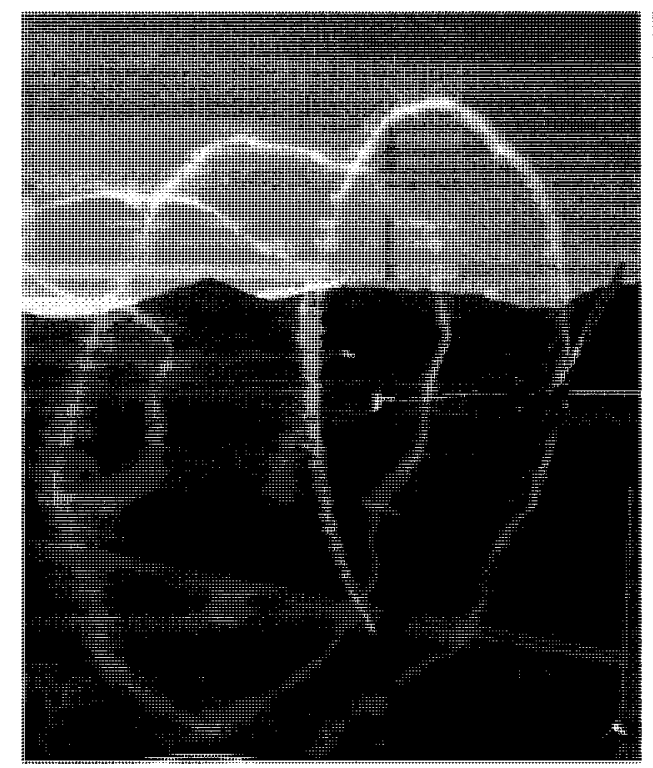

(a) Wake in unsteady wind [43]

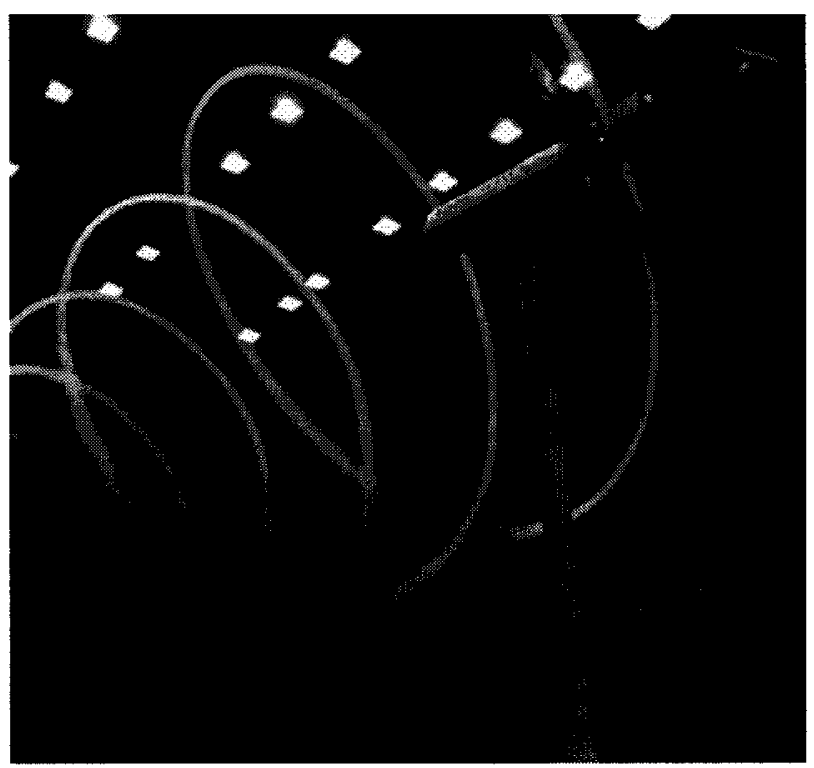

(b) Steady wake in a wind tunnel [43]

Figure 4.6: Wakes behind the NREL turbine in steady and unsteady environments.

Yawed flow conditions can occur on wind turbine rotors due to wind gusts or when small stall-controlled turbines are turned out of the wind to control their power output [44]. New Megawatt-scale commercial turbines all incorporate a yaw control system, but turbine operation in yawed flow can still occur due to sudden changes in wind speed and direction. The turbine rotor yaw misalignment and the skewed wake behind the rotor will create unsteady inflow conditions, leading to unsteady 
turbine blade loading and an unsteady power output. [44]. As the unsteady loading affects fatigue loads and power quality, it is essential for computational techniques to accurately capture the effects of yaw misalignment.

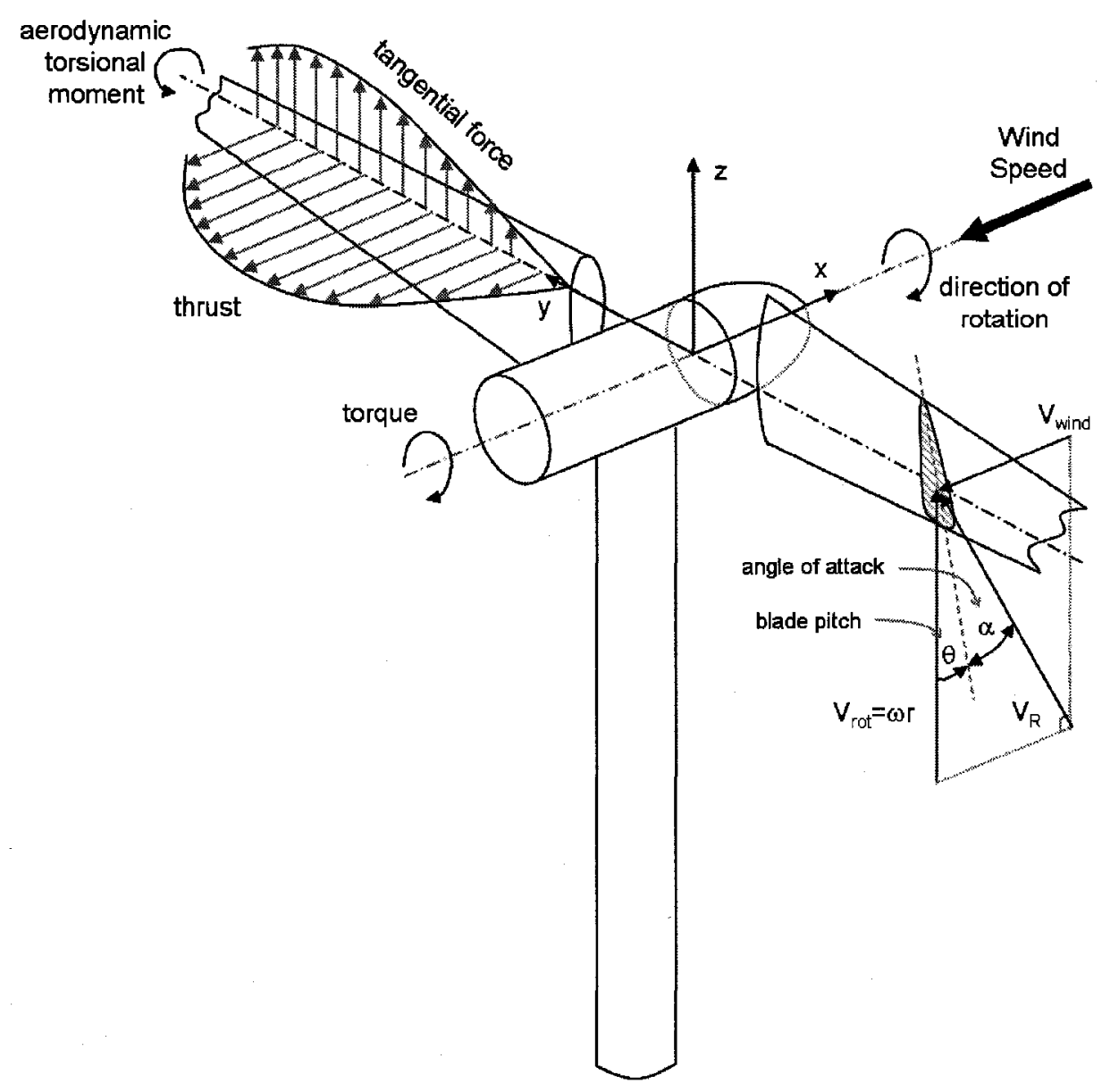

Figure 4.7: Representation of the loads and stresses on a turbine rotor.

\subsection{Wind Farm Effects}

As a turbine extracts energy from the flow, there will be a velocity deficit in the wake behind the turbine relative to the inflow conditions. Whenever turbines are located in a wind farm, the velocity deficit and the amount of spacing between the turbines 
will reduce the amount of power generated by downstream turbines. It has been demonstrated that array losses in wind farms are approximately $15-25 \%$ when compared to the same number of turbines in isolation $[34,45]$. The overall array efficiency is affected by the number of turbines in the farm, the geometry of the farm layout, the wind turbine thrust and power characteristics, the wind turbulence intensity, and the frequency distribution of the wind direction. Based on field measurements, the recommended turbine separation within a farm is 8 to 10 rotor diameters in the prevailing wind direction and 3 to 5 rotor diameters in the cross-wind direction [34]. A downwind separation of 8 to 10 rotor diameters will allow the velocity deficit caused by upstream turbines to recover to a speed closer to the ambient wind speed, but the effects of the turbine wake and the velocity deficit can still be present 20 diameters downstream of the rotor [34].

The behaviour of the turbine power curve in a wind farm is affected by the velocity deficit and by the wind direction. When the wind speed reaches the cut-in speed of a particular turbine, the turbines in the first row of a wind farm will start generating power, but the resulting velocity deficit will delay the startup of downstream rows of turbines. The remaining rows of turbines will start as the wind speed increases. Because of the velocity deficit and the wake behaviour, all of the downstream turbine rows will not reach their rated power until the ambient wind speed is higher than the rated wind speed of a single turbine in isolation [34]. All of these effects will change the shape of the power curve of a turbine in a wind farm, as seen in Figure 4.8. Additionally, should the direction of the wind change from the prevailing wind direction, the separation distance between the rotated turbines is reduced and will lower the power output of the downstream turbines. The decrease in power output in closely spaced situations and the effects of turbine wake interaction in a wind farm must be properly evaluated to develop accurate power prediction estimates. 


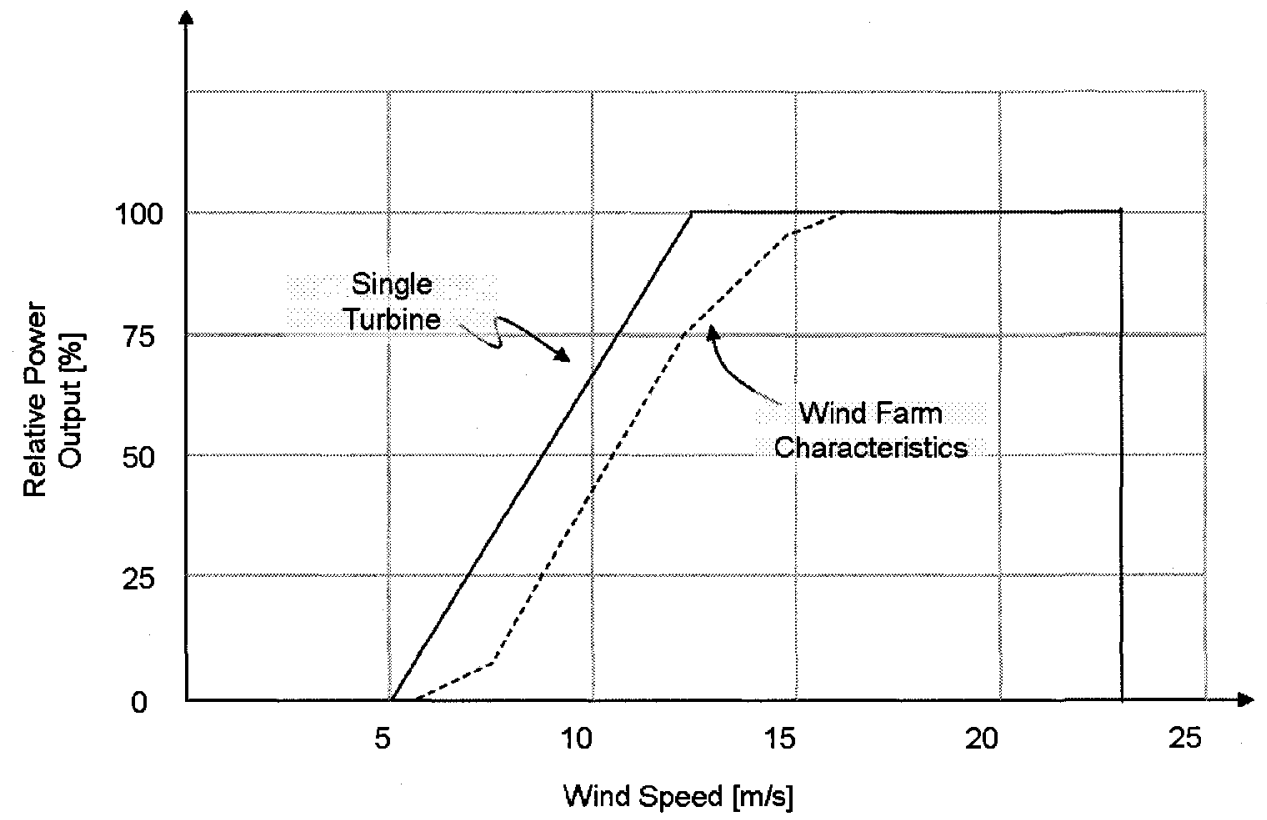

Figure 4.8: Power curve of an isolated turbine and of a wind farm. Adapted from [34]. 


\section{Chapter 5}

\section{A Review of Wind Turbine Aeroelasticity}

The prediction of the annual energy output of a wind turbine or wind farm is directly dependent upon the accuracy of the computational techniques used in commercial or research codes. These techniques consist primarily of blade element methods, actuator disk methods, potential flow methods, and full CFD methods, though a number of empirical wake models have been developed for commercial analysis tools. Wind farm software packages rely on simplified wake modeling to evaluate the effect of the wakes of upstream turbines on downstream rotors. While wake models are generally valid at the conditions under which they were developed, they rely on many empirical assumptions [46].

A thorough review of the aerodynamic techniques that are used to resolve the near and far wakes of wind turbines was published by Vermeer et al in 2003 [42]. This review also summarized the major experimental efforts that have been undertaken to characterize the loads and wakes produced by wind turbines. A follow-up review in 2006 by Hansen summarized the aerodynamic and aeroelastic techniques that are presently being used to study wind turbine loads [47]. Recent experiments have demonstrated that there are many inaccuracies in the prediction of wind turbine and wind farm performance [48]. The assumptions, merits, and inadequacies of the primary wind turbine and wake prediction techniques will be discussed in this Section. 


\subsection{Wind Turbine Wake Modeling}

A number of commercial software packages have been developed to plan wind farm layouts, to calculate annual energy yields, to perform financial analysis, and to predict the wake losses that occur in wind farms. Proper characterization of turbine wakes is essential for accurate energy predictions in order to both determine the wind velocity deficit at downstream turbines and to accurately capture the physics of the flow around a single rotor. All commercial wind farm programs contain a database of wind turbine thrust and power coefficients that can be imported from existing designs or determined from aerodynamic solvers in advance.

\subsubsection{Wake Models}

The PARK wake model, developed by Katic et al [49], is two-dimensional model that uses momentum theory to predict the initial velocity deficit behind a turbine. A rectangular velocity profile is initiated two diameters downstream of the turbine and the wake is assumed to expand linearly downstream according to an empirical wake decay constant, as seen in Figure 5.1. As the centerline of the wake follows the terrain in the model, the mapped terrain must be relatively homogeneous to approximate the physical behaviour of the wake. The PARK model, though very rapid, does not properly represent the physical behaviour of the wake and relies on momentum theory, which is invalid in many turbine loading conditions.

In the late 1980s, Ainslie developed a wind turbine wake model that formed the basis for the models that have been implemented in the wind farm software GH Windfarmer $[51,50]$ and FLaP [52]. The Ainslie model is a semi-empirical wake model that is based on the discretization of the inviscid thin layer Navier-Stokes equations and is depicted in Figure 5.2.

In the Ainslie model, it is assumed that the flow is axisymmetric, fully turbulent, 


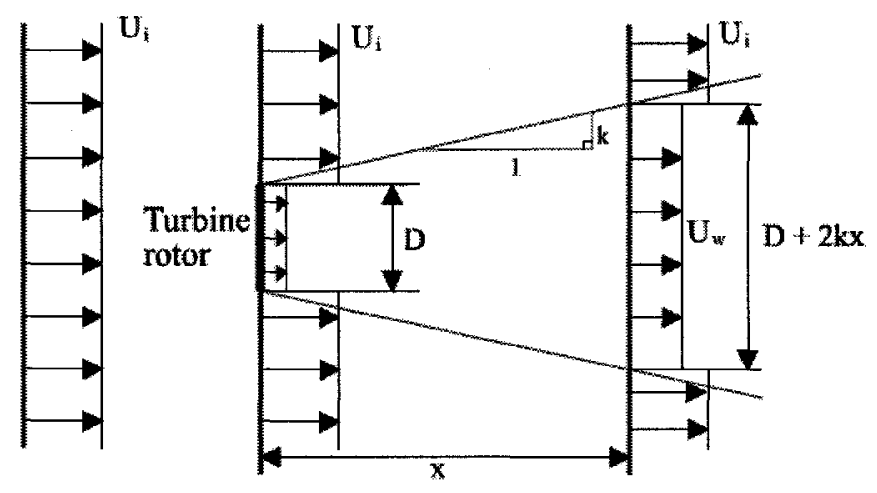

Figure 5.1: Schematic of the PARK wake model. Reproduced from [50].

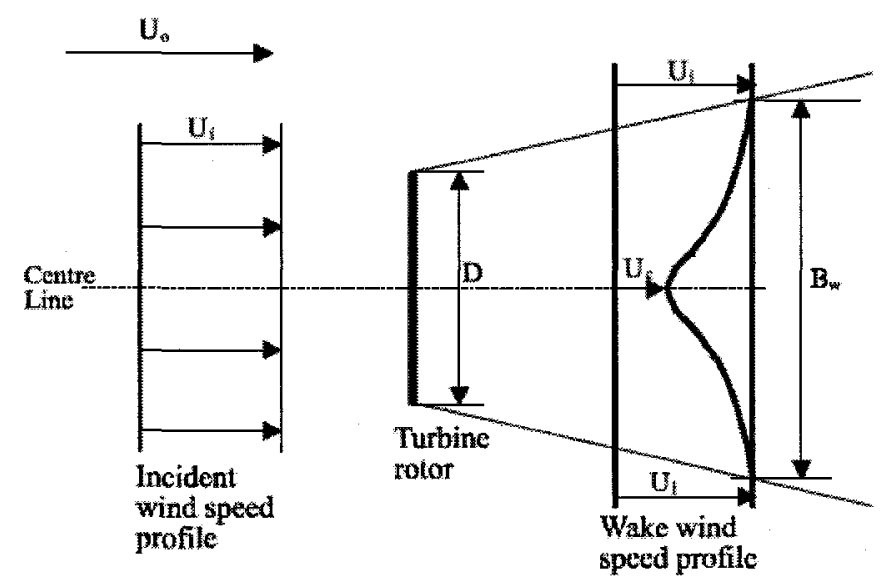

Figure 5.2: Schematic of the GH Eddy Viscosity (Ainslie) wake model. Reproduced from $[50]$.

that there are no circumferential velocities (no swirl) and that the external flowfield is steady in time. The solution for the initial wake is initiated $2 \mathrm{D}$ downstream from the turbine and the initial profile has a Gaussian shape that depends on the turbine thrust coefficient, the ambient turbulence intensity, and the estimated wake width [51]. Pressure gradients in the fluid outside the wake are neglected, and axial pressure gradients are neglected in the wake, as after the near wake region, the gradients of mean quantities in the radial direction are larger than gradients in the axial direction [51]. There are several empirical elements to the model that represent the behaviour of the near wake region, the shear layer behaviour, and wake meandering due to 
topographical effects [53]. A detailed technical treatment of the model can be found in $[50,51]$.

\subsubsection{Wind Farm and Wake Modeling Software}

Wind farm modeling software is used to predict the energy production of a wind farm. The accuracy of current software packages is dependent on existing turbine power curves, simplified aerodynamic methods, and empirical wake models.

RETScreen is an energy management software package that was developed by Natural Resources Canada [54]. This MS Excel-based software has been designed to conduct pre-feasibility and feasibility studies of clean energy technologies. The modeling of a wind turbine in RETScreen is based on a database of turbine power curves. Though a general loss coefficient can be specified for turbine arrays, RETScreen does not physically model the wake behind a turbine, and does not consider yaw behaviour or terrain effects on turbine output [54].

The Wind Atlas Analysis and Application Program (WAsP) was developed by the Wind Energy Department at Ris $\varnothing$ DTU in Denmark and has become one of the industry standard wind energy applications [55]. The program is used for wind resource mapping and assessment, climate estimation, wind turbine siting, and for estimating wind farm power production. The wake model that is used in WAsP is based on the PARK model developed by Katic et al [49] and its implementation in WAsP is described in [55]. The limitations of the wind model and terrain treatment in WAsP have been well documented in literature $[56,57]$.

GH Windfarmer, developed by Garrad-Hassan Ltd in Bristol, UK, is an analysis package designed to predict the power output of a wind farm. GH Windfarmer can implement either the PARK model of Katic [6] or an eddy-viscosity wake model based on the work of Ainslie [51]. The Wind Farm Layout Program FLaP was developed at the University of Oldenburg in Germany in 1993 and is used to design and optimize 
wind farm layouts and similar to GH Windfarmer, models turbine wakes using the Ainslie model [58].

ECN Wakefarm is a wind farm analysis package developed at the Energy Research Centre of the Netherlands that is based on the single-turbine UPMWAKE code developed at the Universidad Polytecnica de Madrid [59]. Wakefarm uses blade element momentum theory to calculate the axial induction factor and the thrust coefficient of a turbine. In 2006, several improvements were made to Wakefarm to more accurately reflect the physical nature of the wake. In the new version of the code, the axial pressure gradient is prescribed as an external force instead of being neglected and wake expansion is enforced as well [59]. The pressure gradient is calculated a priori using an axisymmetric vortex tube solution. This potential flow method treats the turbine as an actuator disc and uses the axial induction factor determined with the blade element method. Pressure gradients are calculated for a range of induction factors and are stored in a database. During simulations, Wakefarm then determines the pressure gradient for each turbine in the wind farm from a lookup table based on the axial induction factor [59].

\subsubsection{Wake Model Validation}

The GH Eddy Viscosity wake model has been validated against a wind tunnel experiment performed by Ainslie and Hassan in 1989 [53, 46] and using operational data from the Norrekaer Enge II wind farm [46]. The results of the wind tunnel tests demonstrated that the wind speed profiles across the wake behind a single turbine are well predicted with the eddy viscosity model in axisymmetric flow situations where the velocity deficit takes an approximately Gaussian shape. The centerline turbulence intensity was not properly represented at distances less than 7D from the rotor, with predictions ranging from $100-150 \%$ of the measured turbulence intensity [46]. However, results are not shown for the case of waked turbines or the performance of 
the model in yawed flow so it is unclear how the model performs in such conditions.

The Ainslie model was also validated against power measurements taken at the Norrekaer Enge II wind farm in two orthogonal directions. The farm consists of a $6 \times 7$ array of turbines. Though qualitative agreement between the model and the measured power was demonstrated, a difference of $25 \%$ can be observed between the power predictions and the measurements at each row of turbines [46].

The above-mentioned wind farm analysis codes have found widespread use in industry, but have had little independent validation. However, as part of the Efficient Development of Offshore Windfarms (ENDOW) project in Europe, the offshore wake prediction capabilities of five wind farm software packages (including Wakefarm, Windfarmer, and FLaP) were evaluated against measured wake deficit data [60]. Downstream of the rotor, the model errors were approximately $20 \%$ of the measured data, with disagreement between the various models. Additional tests were preformed on interacting wake cases (up to 5 wakes) and increased variability was found between the model predictions and the measured results [60]. The authors noted that the wake superposition assumption used by most models must be improved and that the individual wake calculations must be more accurate [60].

The empirical relationships and simplifications inherent in commercial wake models are inaccurate in many loading conditions, including yawed flow situations and in complex terrain. The results of the ENDOW project have demonstrated that there is a lack of physical characterization of the wake behaviour in commercially available wind farm tools and have highlighted the need for simulations that can accurately represent wind turbine wakes. 


\subsection{Aerodynamic Modeling Techniques}

\subsubsection{Blade Element Momentum Theory}

Blade element methods (BEM) are the simplest analysis tools available for wind turbines, and can perform computations in the order of seconds. They use a combination of blade element theory and momentum theory along with a series of empirical approximations to calculate the loads acting on a turbine blade at a discrete number of radial panels. This theory has been developed from the blade element theory of Glauert in 1935 [61]. While BEMs have been shown to reasonably predict inflow behaviour within a narrow range of axisymmetric conditions [62], they are not physically valid in yawed flow conditions, and are mathematically invalid at typical turbine blade tip speed ratios. As such, there are many empirical relationships that have been developed to approximate tip losses, the effects of a finite number of blades, and yaw corrections that are not based on a physical characterization of the flow.

A detailed derivation of BEMs based on modeling the axial and radial inflow can be found in $[63,64]$, whereas a BEM based solely on the axial inflow to the rotor disk has been described by Leishman [45]. A review of the application of BEM techniques in commercial wind turbine analysis software is presented by Molenaar and Dijkstra in [65]. The authors demonstrate that virtually all commercially available software (the authors present 11 examples, including Adams, Bladed, and Phatas) use BEM, typically coupled with a linear beam aeroelastic module. Molenaar highlights the need to develop more physically representative dynamic models, discusses the lack of accuracy in yawed-flow situations, and is critical of the lack of proof of code validation in commercial packages [65]. Additional information regarding the validity and application of BEM techniques is presented in $[42,47,62]$. 


\subsubsection{Actuator Disk Techniques}

Alternatives to blade element methods are actuator disk or actuator line methods, where the wind turbine is represented as a discontinuous surface or line through which aerodynamic forces can be imposed on the flowfield. The flow field is computed by an Euler or Navier-Stokes CFD solver, and the aerodynamic forces that represent individual wind turbines are then imposed onto the grid of the CFD solver [66]. The theory for actuator disk methods was developed in 1962 by Wu [67], and they have recently been applied to a wide range of problems, including yawed simulations [42], turbine wake interaction studies $[68,69]$, and the response of unsteady flow on a turbine [70].

Though actuator disk methods have been used extensively for research $[42,47]$, the geometry of the turbine blades and viscous flow around the blades are not resolved as only the surface forces are imposed on the general flowfield. The aerodynamic

forces are typically determined with two-dimensional BEMs and are then corrected for three-dimensional effects, and as such, can suffer from the many of the limitations of blade element momentum theory [42].

\subsubsection{Potential Flow Methods}

In potential flow methods, the blade aerodynamics and the wake are typically resolved using a lattice of vortex elements or vortex particles that are shed from the blades. A schematic comparing the representation of the wake of a helicopter with a vortex lattice method to the wake using a vortex particle representation can be seen in Figure 5.3. The trailed vortices are allowed to convect with the freestream and their mutually induced velocities [71].

Potential flow methods can be classified as prescribed-wake methods or free-wake methods. In prescribed-wake simulations, the wake geometry is specified a priori 

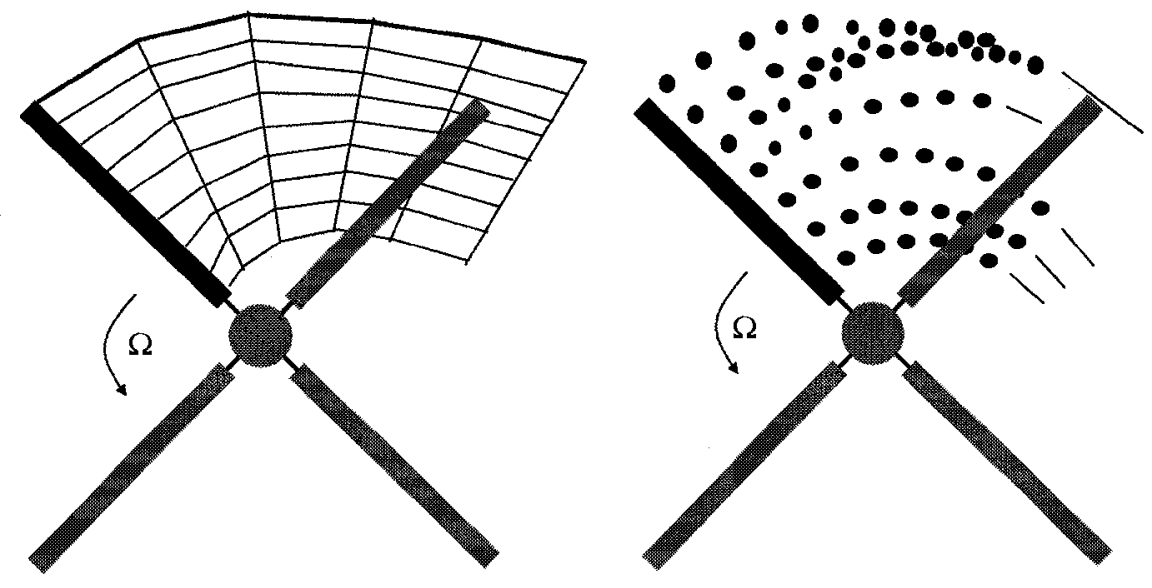

Figure 5.3: a: vortex wake representation with a vortex lattice model. b: vortex wake representation with a vortex particle method. Adapted from [45]

based on wind tunnel results or empirical relationships, whereas the vortex elements in free-wake simulations are allowed to convect freely with the flow [42]. In prescribed wake models, the spatial development of the wake is defined using geometry prescription functions and is not deformed as a result of self-induced velocities. The shape of the developed wake is typically obtained only by considering the induced velocities of the rotor blades [71]. In free-vortex models, however, the wake can be either created by successive vortex filament shedding, or by performing a number of iterations on a complete preset wake geometry. In both cases, the wake deformation is determined by the self-induced velocities of the vortex filaments as well as the induced velocity of the rotor blades [71]. Prescribed-wake models have been used by several authors $[47,71,72,73,74]$, whereas free-wake models have not been commonly used to analyse wind turbine wakes [62], though they have found acceptance in the rotorcraft research community [45].

One currently-used free-vortex method is the Rotor Vortex Lattice Method (ROVLM), developed at the University of Stuttgart, in which the rotor and wake are treated as singularity solutions of the Laplace equation $[75,76]$. Dipoles are placed at the rotor blades to simulate lift forces. The wake flow is assumed to be inviscid 
and irrotational. Vorticity is shed into the wake as a spanwise series of panels from the trailing edge, where the amount of shed vorticity is determined by the Kutta condition [25]. In each time step, a new portion of the wake is created and convected downstream. The wake deformation is determined by multiplying the wake panel velocities with the time step and a simplified wake transport model is used for the far wake [75]. ROVLM was used in a study by Whale et al in 2000 to experimentally and numerically study the vortex structure of a model turbine in a wind tunnel [76]. It was found that the numerical results underpredicted the wake expansion that was visible in the experiment. Additionally, ROVLM overpredicted the strength of the shed vortices at the blade root, a problem that the authors attributed to the stall model that was included in the code [76].

In 2003, Duque et al tested a vortex lattice code, CAMRAD II, against the NREL's measured data [77]. CAMRAD II was originally developed for rotorcraft and has been modified for horizontal axis wind turbines and consists of a vortex lattice with a freewake model. The solver requires $2 \mathrm{D}$ lift and drag data, corrected for stall delay effects caused by the effect of the Coriolis force effect on the spanwise momentum in the boundary layer. Results for CAMRAD II with a stall delay model were within $10 \%$ of the measured power until the freestream velocity increased beyond $15 \mathrm{~m} / \mathrm{s}$, after which a large over prediction of power was observed, increasing to an error of $50 \%[77]$.

A more recently developed free-wake vortex code is that developed at the University of Maryland by Gupta and Leishman [36]. Each blade is modeled using a Weissinger-L lifting surface model, which is a single panel lifting surface. The blades are separated into a number of spanwise segments over which bound circulation and lift are constant. The near wake is truncated after an angular rotation of 30 degrees and a single vortex filament is then trailed from the tip of each blade by assuming that the bound vorticity rolls up into the tip vortex. The results of this work have 
been published by Gupta and Leishman in a number of papers $[44,62,78,79]$ and have been validated in yawed and unyawed conditions using several experiments $[48,80]$. The numerical results of this code are promising, though the inadequacies in the stall delay models that are currently being used have been noted in [36]. This particular free-wake code has not yet been coupled with a structural dynamics model.

Two other early free-wake codes for wind turbines were developed in the late 1980 s, but do not appear to have been used recently $[81,82]$.

\section{Discrete Vortex Methods}

A particular class of free-vortex methods are discrete vortex methods (DVM). The General Unsteady Vortex Particle (GENUVP) code, developed by Voutsinas et al in Greece [83], models unsteady, incompressible, inviscid, and rotational flows. A vortex particle approximation (also referred to as vortex blobs in literature) of the free vorticity is used to simulate the behaviour of the wake and a dipole approximation is used for the flow induced by the turbine blades. Vortex particles are shed from spanwise strips along the trailing edge of a blade as well as from the chordwise elements along the blade tip. The code has been validated against the Tjaereborg turbine [83], participated in the NREL blind comparison [48], and forms the aerodynamic basis for SMARTROTOR [3], the aeroelastic discrete vortex method developed at Carleton University and used in the present work.

SMARTROTOR was developed at Carleton University in 2002 as an aeroelastic, aeroacoustic code for rotorcraft analysis and combines a discrete vortex particle method to simulate the wake, a nonlinear structural dynamics model to simulate blade deflection, and a variation of the Ffowcs Williams-Hawkings acoustic model to characterize blade aeroacoustics. SMARTROTOR has recently been used to properly characterize several aeroelastic and rigid-body helicopter problems $[84,85,86]$ and will be validated for use with wind turbines in this work. A complete description of 
SMARTROTOR will be provided in Section 6.1.

\subsubsection{Computational Fluid Dynamics}

There has been a recent movement to apply CFD techniques to study wind turbine aerodynamics. Viscous, incompressible CFD solvers are particularly well-suited to the operational regime of wind turbines and there have been several promising demonstrations of CFD in rotary-wing aerodynamics $[77,87]$. However, the very large computational requirements of rotary-wing simulations, the effects of numerical dissipation in the turbine wake, and the limitations of a grid-based solution in resolving the wake have limited the applicability of CFD in the wind energy industry.

As part of the blind comparison test of the NREL wind tunnel experiment, Sørensen et al used the EllipSys3D CFD solver, an incompressible Navier-Stokes solver code developed at Ris $\emptyset$ [87], to perform steady and unsteady computations. In these simulations, the tower and nacelle were neglected, zero vertical shear was assumed and only axisymmetric flow was considered. As such, it was assumed that the blade will see the same inflow characteristics irrespective of azimuthal rotor position and the rotor was modeled with 1 blade using periodic boundary conditions to account for the second blade [87]. Over 3 million cells were used to model the rotor and freestream. In the unsteady simulation, 830 time steps per revolution were modeled for 3.4 revolutions, requiring 40.8 hours on 4 CPUs. As six different test conditions were run, a grid convergence study could not be performed as the tests required too much time [87]. The calculated rotor torque closely matched the measured NREL experimental results (overpredicted prior to stall onset and underpredicted in deep stall conditions) [87]. There was no discussion regarding the behaviour of the wake or the role of numerical dissipation in the solution. This code, however, produced the most consistently accurate predictions during the NREL blind comparison [48].

In 2003, Duque et al tested a RANS code, OVERFLOW-D2, against the NREL's 
measured data [77]. OVERFLOW-D2 solves an implicit formulation of the compressible RANS equations with overset grids, using 11 million grid points [77]. The solver used a one equation Baldwin-Barth turbulence model. In axial flow simulations, 5000 iterations were performed using 8 CPUs and requiring 26 hours, but the number of revolutions is not presented for axial flow. In yawed flow however, 7200 steps per revolution were performed, requiring 48 hours with 8 CPUs. The results of the Navier-Stokes solver were within $10 \%$ of the measured results when comparing the output power as a function of wind speed [77].

A more recent study was performed by Sezer-Uzol et al in 2006 using an inviscid finite volume CFD code (PUMA2) to simulate the NREL 2-bladed rotor [88]. An unstructured, rotating grid composed of 3.6 million tetrahedral cells was meshed around the turbine blades, but the tower and nacelle were not modeled. The simulation time required to perform 1 revolution with 90,000 iterations ranged from 1.7 days with 128 processors to 15 days with 16 processors. The results of the study demonstrated that the tip vortices dissipated much too rapidly, particularly in yawed flow [88]. It was suspected by the authors that the grid orientation contributed to the rapid vortex dissipation, as the grid had been refined for a $0^{\circ}$ yaw case but was used for a $30^{\circ}$ yaw case in which an asymmetric wake develops. Additionally, the authors cited numerical dissipation as a reason for the inaccurate tip vortex modeling [88].

In 2007, Massouh et al compared PIV measurements of a 3-bladed turbine model with RANS simulations using Fluent [89]. The CFD simulation used six million cells, a sliding mesh and did not apply rotational periodicity in order to perform yawed simulations. It was found that the CFD-predicted vortex core radii were smaller and the distance between vortex cores was greater than measured in the experiment, with weaker vortices that dissipated more rapidly than the experimental observations [89].

Another challenge in RANS CFD modeling is the selection and behaviour of turbulence models. In 2003, Benjanirat and Sankar studied four different turbulence 
models (Baldwin-Lomax, Spallart-Almaras, $k-\epsilon$ without wall functions, and $k-\epsilon$ with wall functions) using the NREL rotor [90]. It was found that the prediction of chordwise forces, torque generation, and $1 / 4$ chord pitching moment were sensitive to the modeling of near wall effects and separated flow effects. The authors recommended that additional work be performed on transition modeling for wind turbine turbulence models [90]. Additional results were presented in 2005 by Tongchitpakdee, Benjanirat and Sankar concerning an unsteady RANS simulation on the NREL turbine in yawed flow [91]. A single CPU required 26 hours for 2 rotor revolutions and the authors highlighted the sensitivity of the results to the selected turbulence model and the manner in which transition is predicted.

As demonstrated, the use of CFD to model full wind turbine rotor configurations is computationally demanding, and simplifications are frequently made (neglecting the tower and nacelle) to simplify the meshing and computational requirements. CFDbased methods have demonstrated the ability to properly predict several important features, but there are several challenges to overcome: the solution is dependent on the quality of the grid (computational requirements are prohibitive to grid convergence studies), there is grid-orientation sensitivity in yawed-turbine simulations, there is rapid numerical diffusion in the wake, and the high computational overhead only allows simulations to be performed for 2-4 rotor revolutions.

\subsubsection{Hybrid CFD/DVM Analysis}

An alternative to potential flow vortex techniques and RANS CFD techniques is the recent emergence of hybrid CFD-potential methods. In these techniques, a small region around the turbine blade is modeled with a computational mesh and CFD simulations are performed to properly capture the three-dimensional effects of the flow on the rotor blade without the need for stall delay models, dynamic stall models, or the use of two-dimensional airfoil data. The vorticity in the flow is then trailed 
from the Navier-Stokes region using a vortex-wake potential flow technique.

A Parallelized Coupled Solver was developed by Schmitz and Chattot at the University of California [74] for wind turbine applications. This solver used a RANS mesh around the turbine blades and a prescribed vortex method in the far field. The vortex line method used a lifting line model with a rigid wake. CFX was used with a $\mathrm{k}-\omega$ turbulence model to perform the CFD calculations in a zone that extended 1-3 chord lengths around the blade and 3-6 chord lengths into the wake. This approach required 800,000 CFD grid nodes which were coupled to the potential flow solution. The Parallelized Coupled Solver accurately predicted the peak torque for the NREL experiment and the authors presented an insightful analysis of the threedimensional effects that occur on the blades with respect to stall delay and tip vortex formation [74].

Another hybrid code was developed at the Georgia Institute of Technology in 2001 by $\mathrm{Xu}$ [92] and additional results are published in [93]. Vorticity is captured by a RANS solver and is then carried into the near and far wake as a vortex filament emanating from the tip of the blade. A prescribed wake model is used in this instance to determine the wake shape, which assumes the radius of the rotor [92]. It was demonstrated in [93] that the hybrid code results were less accurate than full RANS simulations when simulating the NREL turbine.

\subsection{Structural Modeling Techniques}

A complete review of structural modeling techniques for wind turbines was given by Hansen in 2006 [47]. Structural dynamics models have been created for the turbine blades in addition to complete turbine configurations, where the rotor, hub, generator, nacelle and tower are all modeled. In the case of offshore wind turbines, additional water loads must be considered. Solvers typically use either modal analysis or a finite 
element approach to model wind turbine dynamics, but most commercial aerodynamic codes use an FEM approach [47]. A review of the modeling techniques available in a variety of commercial wind turbine codes is presented by Molenaar [65].

Almost all structural FEM models of wind turbines are based on classical onedimensional beam theory $[47,94,95]$ due to the high rigidity of wind turbine blades compared to helicopter rotor blades. As wind turbine designs increase in size and as designs become more flexible, non-linear rotor blade modeling will however become necessary $[47,96]$.

\subsection{Motivation for the current work: The NREL Experiment}

The NREL Unsteady Aerodynamics Experiment Phase VI was performed throughout 1999 and 2000 using a $10 \mathrm{~m}$ diameter wind turbine installed in the NASA Ames $24 \mathrm{~m}$ $\mathrm{x} 36 \mathrm{~m}$ wind tunnel, seen in Figure 5.4. A twisted, two-bladed, stall-controlled rotor was tested and 14 different turbine configurations were analyzed. A large database of measurements was produced, which has been used to validate many recent computational efforts. A summary of the experimental results can be found in [48], where it is demonstrated that the test instrumentation produced highly repeatable, high quality measured data. A complete description of the test plan, model geometry, instrumentation, and calibration procedures can be found in [97]. A blind code comparison was performed as part of the experiment with 30 researchers from 18 participating universities, government laboratories, and industrial partners in Europe and the United States. Participants were provided with the model geometry and test conditions, but did not have access to the test results until they had submitted their respective model's predictions. The codes that were tested ranged from blade element models, 


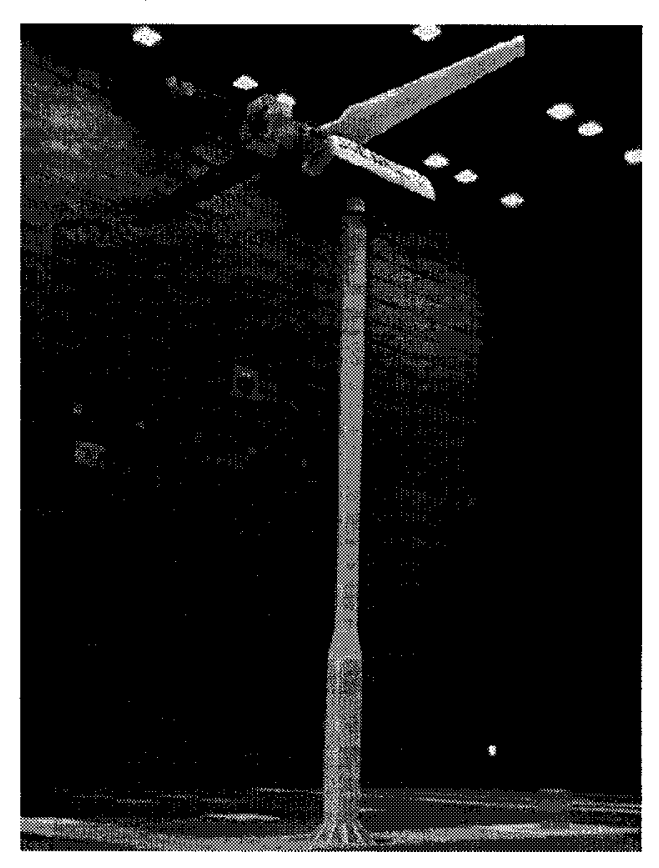

Figure 5.4: The NREL $10 \mathrm{~m}$ diameter wind turbine [43].

prescribed-wake models, free-wake models, and full Navier-Stokes codes. A summary of the participants and their modeling software can be found in [48].

The published results of the comparison demonstrated significant amounts of variability in prediction accuracy, little consistency between codes, and a limited ability to capture the aerodynamic effects occurring on the turbine $[43,48]$. In $0^{\circ}$ yaw, steadystate, unstalled conditions, the power predictions ranged from $25 \%$ to $175 \%$ of the measured data and at higher wind speeds, where dynamic stall and unsteady blade loading is present, the predicted power was $30 \%$ to $275 \%$ of the measured data [48]. This variability is demonstrated in Figure 5.5 which presents the predicted torque output (proportional to the power output of the turbine) of 8 of the 18 participants. It was found that only one code consistently predicted the measured results accurately - the EllipSys3D incompressible Navier-Stokes solver from Ris $\varnothing$ [87]. However, each simulation with this particular computational code required days to perform and yawed-flow cases could not be considered at all [48]. Based on the results of the full 


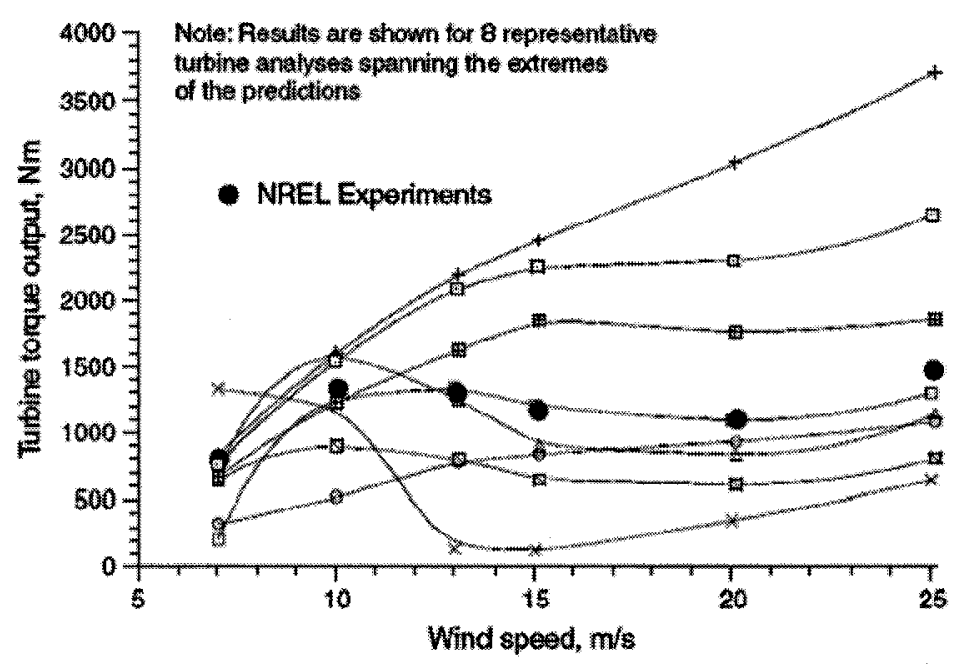

Figure 5.5: Predicted and measured turbine torque from the NREL blind comparison. [41].

scale NREL experiment, it is clear that the accuracy of present aerodynamic models and codes is insufficient and that the assumptions and empiricism present in many cases do not physically represent the nature of a wide variety of problems. A number of areas that require investigation were highlighted in $[41,48]$ and include studying the effects of dynamic stall, post-stall behaviour and properly characterizing the wake created by the turbine.

Carleton University's aeroelastic discrete vortex method code, SMARTROTOR, presents a unique opportunity to study turbine wake behaviour and the wake's effect on wind turbine performance. The grid-free nature of the wake and the threedimensional nature of the code allows accurate computation of the power generated by a turbine in low to medium wind speeds. Additionally, turbine performance in yawed flow situations can be conducted without a reliance on empirical corrections or a CFD grid. Due to the relatively quick computational capabilities of the code, turbine wake interaction studies can be performed to better understand wind farm aerodynamics. Additionally, the structural model implemented in SMARTROTOR can offer increased accuracy in aeroelastic simulations. 


\section{Chapter 6}

\section{Aerodynamic and Aeroelastic Theory in SMARTROTOR}

SMARTROTOR is a state-of-the-art three-dimensional aeroelastic code developed specifically for rotary-wing applications. Its aerodynamic model is based on a discretevortex method called GENUVP while the structural model is based on an implementation of Hodges' nonlinear beam model for rotorcraft. A summary of the theory for these two models will be reviewed in this chapter.

\subsection{Aerodynamic Model}

The aerodynamic component of SMARTROTOR is the GENeral Unsteady Vortex Particle (GENUVP) software originally developed by Voutsinas et al at the National Technical University of Athens [83]. GENUVP uses an unsteady panel method cou-

pled to a discrete vortex particle method to resolve the rotor blade and wake aerodynamics. 


\subsubsection{GENUVP Theory}

A detailed description of the theory implemented in GENUVP can be found in [83, $98,99,100]$, but the the theory will be summarized in this section for completeness.

The theory for aerodynamic modeling in GENUVP is based on the Helmholtz decomposition, or vorticity transport theorem. The Helmholtz decomposition states that any vector field can be written as the sum of a solenoidal (rotational, or divergence-free) and lamellar (irrotational, or curl-free) vector [101]. The Helmholtz decomposition of an arbitrary velocity field $u$ is expressed as

$$
u=v+\nabla \alpha+\nabla \times \beta
$$

where $v$ is the harmonic vector field, $\alpha$ represents a scalar potential, and $\beta$ represents a vector potential. In the context of a wind turbine, the irrotational component of the flow represents the influence due to the solid boundaries and the rotational component of the flow represents the influence of the wake. The velocity field around a helicopter or wind turbine can be represented with the Helmholtz decomposition as

$$
\vec{u}(\vec{x}, t)=\vec{u}_{\text {external }}(\vec{x}, t)+\vec{u}_{\text {solid }}(\vec{x}, t)+\vec{u}_{\text {near-wake }}(\vec{x}, t)+\vec{u}_{\text {far-wake }}(\vec{x}, t)
$$

where $\vec{x}$ and $t$ are the position and time, respectively, of the velocity $\vec{u}(\vec{x}, t)$ in a domain $D$. The irrotational component of the flow in Equation 6.2 is composed of the the prescribed external velocity field, $\vec{u}_{\text {external }}$, the velocity field due to the influence of solid bodies (such as the rotor blades, the nacelle, or tower), $\vec{u}_{\text {solid }}$, and the velocity induced by the near-wake of the lifting surface, $\vec{u}_{\text {near-wake }}$. The rotational component of the flow is represented by the induced velocity due to the far-wake of the rotor blades, described in Equation 6.2 by $\vec{u}_{\text {far-wake }}$. 
In GENUVP, singularity distributions are defined on all solid surfaces and the near wake, whereas the far-wake behind the lifting surface is represented with free-vortex particles [98]. The far wake velocity, $\vec{u}_{f a r-w a k e}$, induced at a location in space $(\vec{x})$ by the vorticity of a particle $(\vec{\omega})$ located at a point $\left(\vec{x}_{o}\right)$ is given by the Biot-Savart law, as seen in Equation 6.3.

$$
\vec{u}_{f a r-w a k e}(\vec{x}, t)=\int_{D_{w}(t)} \frac{\vec{\omega}\left(\vec{x}_{o}, t\right) \times\left(\vec{x}-\vec{x}_{o}\right)}{4 \pi\left|\vec{x}-\vec{x}_{o}\right|^{3}} d D
$$

The far wake velocity described by Equation 6.3 can be solved with vortex methods that describe the vorticity in the field [3]. As such, Equation 6.2 can be solved using a panel method for the $\vec{u}_{\text {solid }}$ and $\vec{u}_{\text {near-wake }}$ terms and a vortex method for $\vec{u}_{f a r-w a k e}$. As the fluid is inviscid, there is no automatic link between the irrotational and rotational components of flow [98]. The panel method and the vortex method are coupled at the interface between the near-wake and far-wake by enforcing the Kutta condition at the near wake.

\subsubsection{Panel Method - Solving for $\vec{u}_{\text {solid }}$ and $\vec{u}_{\text {near-wake }}$}

A panel method was implemented in GENUVP based on the work of Hess [102] in order to determine the induced velocity in the flowfield from solid lifting and nonlifting bodies. Dipole distributions are used to generate lift on solid boundaries and surface vorticity on the near wake sheets, whereas sources are used to account for the thickness of lifting and non-lifting bodies. This can be illustrated by considering a body with a surface $S$ in an incompressible, inviscid flowfield. The continuity equation in an inviscid incompressible flowfield reduces to the Laplace equation as described by Equation 6.4, where $\phi$ is the velocity scalar potential.

$$
\nabla^{2} \phi=0
$$


The solution for the velocity potential must satisfy the condition of non-penetration of solid boundaries and the condition of regularity, as described by Equations 6.5 and 6.6 , respectively. The non-penetration boundary condition results in tangential flow at the surface and the condition of regularity ensures that the velocity approaches the freestream speed at infinity.

$$
\begin{gathered}
\left.\nabla \phi \cdot \vec{n}\right|_{S}=\left.\frac{\partial \phi}{\partial n}\right|_{S}=\left(\vec{u}_{e x t} \cdot \vec{n}-F\right)_{S} \\
|\nabla \phi|_{\infty} \rightarrow 0
\end{gathered}
$$

In Equation 6.5, $\vec{n}$ is the unit normal vector on the surface $S$ and $F$ is the local normal velocity caused by the motion of the body and velocity induced by the farwake. Green's identity can be used to write the general solutions of Laplace's equation (Equation 6.4) as source and dipole distributions over the surfaces of the bodies [3]. The potential caused by a non-lifting body (a turbine tower, nacelle, or hub) is determined with a continuous source distribution over the surface of the body, whereas the potential caused by a lifting surface (rotor blade or wing) is determined from a dipole distribution.

The potential induced at a point $\vec{P}$ due to the presence of a non-lifting body is described by Equation 6.7, where $\sigma$ is the local source intensity distribution and $r$ is the distance to the point $\vec{P}[102]$.

$$
\phi(\vec{P})=\int_{S} \sigma\left(\frac{1}{r}\right) d S
$$

A dipole distribution can be used to determine the potential due to thin lifting body, but a source and dipole distribution must be used to model a thick lifting body. While GENUVP has the ability to model thin and thick lifting surfaces, only thin 
lifting bodies are considered in this discussion and the ensuing analysis. A dipole distribution will create circulation around the body resulting in a net lift force [3]. The near-wake is also determined using a dipole distribution trailed downstream of the lifting surface, and must satisfy Kelvin's theorem, which states that circulation must be conserved [23]. The potential induced at a point $\vec{P}$ due to the dipole distribution on a lifting surface or near-wake surface is described by Equation 6.8, where $\mu$ is the local dipole intensity on the surface.

$$
\phi(\vec{P})=\int_{S} \mu \vec{n} \cdot \nabla\left(\frac{1}{r}\right) d S
$$

The potential induced by the lifting and non-lifting surfaces at a point $\vec{P}$ can be determined by expressing Equations 6.7 and 6.8 as

$$
\phi(\vec{P})=-\frac{1}{4 \pi} \int_{\text {lifting }} \mu \vec{n} \cdot \nabla\left(\frac{1}{r}\right) d S-\frac{1}{4 \pi} \int_{\substack{\text { Non- } \\ \text { Lifting }}} \sigma\left(\frac{1}{r}\right) d S-\frac{1}{4 \pi} \int_{\text {Wake }} \mu \vec{n} \cdot \nabla\left(\frac{1}{r}\right) d S
$$

Though Equations 6.7 and 6.8 satisfy the regularity condition described by Equation 6.6, the non-penetration boundary condition must be met by combining Equation 6.5 with Equation 6.9.

$$
\begin{gathered}
\left(-\frac{1}{4 \pi} \int_{\text {lifting }} \mu \nabla\left[\frac{\partial}{\partial n}\left(\frac{1}{r}\right)\right] d S-\frac{1}{4 \pi} \int_{\substack{\text { Non- } \\
\text { Lifting }}} \sigma \nabla\left(\frac{1}{r}\right) d S\right. \\
\left.-\frac{1}{4 \pi} \int_{\text {Wake }} \mu \nabla\left[\frac{\partial}{\partial n}\left(\frac{1}{r}\right)\right] d S\right) \cdot \vec{n}=\vec{u}_{\text {ext }} \cdot \vec{n}-F
\end{gathered}
$$

Equation 6.10 is the governing equation for the panel method implemented in GENUVP and is evaluated at all body surfaces. Equation 6.10 can be approximated 
as a linear system of equations by discretizing the lifting and non-lifting surfaces. Each discretized panel will have a constant source intensity $\sigma$ (for non-lifting bodies) or dipole intensity $\mu$ (for lifting surfaces and the near-wake) [3]. The source and dipole intensities can be moved out of the integrals in Equation 6.10 as they are constant over each panel. The resulting integrals are only dependent upon the surface geometry and the selected discretization. If the integrals are evaluated at the control points of the elements, Equation 6.10 can be approximated with a linear system composed of influence coefficient matrices, expressed as

$$
\begin{gathered}
{\left[C_{i j}^{\text {Lifting }}\right]\left\{\mu_{j}\right\}+\left[C_{i k}^{\text {Near-Wake }}\right]\left\{\mu_{k}\right\}+\left[C_{i l}^{\text {Non-Lifting }}\right]\left\{\sigma_{l}\right\}=\left\{\vec{u}_{e x t} \cdot \vec{n}_{i}-F_{i}\right\}} \\
i=1,\left(N_{\text {Lifting }}+N_{\text {Non-Lifting }}\right) \\
j=1, N_{\text {Lifting }} \\
k=1, N_{\text {Near-Wake }} \\
l=1, N_{\text {Non-Lifting }}
\end{gathered}
$$

where $N_{\text {Lifting }}, N_{N o n-L i f t i n g}$ and $N_{\text {Near-Wake }}$ refer to the number of panels on the lifting, non-lifting, and near-wake surfaces, respectively.

The Kutta condition, which states that there can be no pressure jump at the trailing edge of a thin lifting surface, must be met in order to obtain a unique solution to the linear system described by Equation $6.11[23,98]$. The dipole intensity of the near-wake strip elements are set to equal the value of the adjacent emitting panels along the trailing edge of the lifting surface and along the chordwise panels at the tip of the lifting surface. The near-wake geometry is then determined from the velocity of the trailing edge and tip panels [3]. Equation 6.11 can be solved for the dipole and source distributions along the bodies and near-wake and then the scalar velocity 
potential can be calculated at any point using Equation 6.9. The velocity distribution is calculated using Equation 6.2, where the velocity at a panel can be written as

$$
\vec{u}_{\text {panel }}=\vec{u}_{\text {solid }}(\vec{x}, t)+\vec{u}_{\text {near-wake }}(\vec{x}, t)=\nabla \phi
$$

The pressure distribution on the solid bodies can then be determined with the unsteady Bernoulli equation, as seen in Equation 6.13.

$$
C_{P}=1-\frac{u^{2}}{u_{r e f}^{2}}-\frac{2}{u_{r e f}^{2}} \frac{\partial \phi}{\partial t}
$$

After the pressure distribution has been determined over the lifting bodies, the potential forces acting on the lifting surfaces can be determined. As the panel method is inviscid, the viscous part of the loading is superimposed to account for drag and the effects of stall. An a posteriori scheme is used in GENUVP based on classical strip theory where the radial distribution of the effective angle of attack is determined using the potential forces [98]. The effective angle of attack is used in a look-up table at each spanwise strip to determine the lift, drag, and pitching moment coefficients for the airfoil in question.

\subsubsection{Discrete Vortex Method - Solving for $\vec{u}_{f a r-w a k e}$}

In any vortex method, the wake structure caused by the lifting surfaces is convected through the domain. As mentioned in Section 5.2.3, the wake can be resolved using a vortex lattice method, tip vortex filaments, or with vortex blobs. GENUVP uses a vortex blob (i.e. vortex particle) approach to model the far-wake, based on the work by Rehbach [103]. Therefore, instead of calculating the geometry of vortex sheets and their doublets in the far wake, the evolution of free-vortex particles is tracked throughout the wake [98]. A general discussion of the adaptive, stable, convergent 
nature of vortex blobs is given in [104]. In vortex particle methods, the wake is represented by discrete particles that each contain information regarding their intensity, velocity, and position. The intensity and position of the $\mathrm{j}$-th vortex particle within a domain $D_{\omega}(t)$ can be written as $\vec{\Omega}_{j}(t)$ and $\vec{Z}_{j}(t)$, where the intensity is defined by Equation 6.14

$$
\vec{\Omega}_{j}(t)=\int_{D_{w, j}} \vec{\omega}(\vec{x}, t) d D
$$

where

$$
\begin{gathered}
\vec{\omega}(\vec{x}, t)=\sum_{j} \vec{\Omega}_{j}(t) \delta\left(\vec{x}-\vec{Z}_{j}(t)\right) \\
\vec{\Omega}_{j}(t) \times \vec{Z}_{j}(t)=\int_{D_{w, j}} \vec{\omega}(\vec{x}, t) \times \vec{x} d D
\end{gathered}
$$

The Biot-Savart law for the induced velocity from the far-wake with discrete vortex particles can then be written as

$$
\vec{u}_{f a r-w a k e}(\vec{x}, t)=\int_{D_{w}(t)} \frac{\vec{\Omega}_{j}(t) \times\left(\vec{x}-\vec{Z}_{j}(t)\right)}{4 \pi\left|\vec{x}-\vec{Z}_{j}(t)\right|^{3}}
$$

As discussed by Voutsinas [98], the evolution of a vortex sheet or filament is unstable in time due to the singular nature of the velocity field described by the Biot-Savart law. This can be taken care of by introducing particle (or blob) approximations for the vorticity in the wake with a smoothing function for the velocity field. A smoothing approximation described by Beale and Majda [105] is applied to Equation 6.17 because of its singular nature, described by Equation 6.18

$$
\vec{u}_{f a r-w a k e}(\vec{x}, t)=\int_{D_{w}(t)} \frac{\vec{\Omega}_{j}(t) \times \vec{R}_{j}}{4 \pi \vec{R}_{j}^{3}} f_{\varepsilon}\left(\vec{R}_{j}\right)
$$




$$
\vec{R}_{j}=\vec{x}-\vec{Z}_{j}(t) f_{\varepsilon}\left(\vec{R}_{j}\right)=1-e^{\left(R_{j} / \varepsilon\right)^{3}}
$$

where $\epsilon$ is the cut-off length for the vortex particles. The vortex blobs are convected in the Lagrangian reference frame as defined by Equations 6.19 and 6.20, in which $\vec{D}$ is the deformation tensor.

$$
\begin{gathered}
\frac{d \vec{Z}_{j}}{d t}=\vec{u}\left(Z_{j}, t\right) \\
\frac{d \vec{\Omega}_{j}}{d t}=\left(\vec{\Omega}_{j} \nabla\right) \vec{u}\left(Z_{j}, t\right)=\vec{D} \cdot \vec{\Omega}_{j}
\end{gathered}
$$

\subsubsection{Coupling Between the Near- and Far-Wakes}

The near-wake surfaces are described by panels on which dipole distributions represent the integral of the vorticity around the panel surface [98]. The far-wake however, is represented using a spatial distribution of vorticity with free-vortex particles. Therefore, the wake is treated using two methods at each time step. The coupling between the near-wake dipole distribution and the far-wake free vorticity is shown schematically in Figure 6.1. At each time step, the dipole strength of the near-wake panels (shaded in grey) will be evaluated. In the ensuing time step, the near wake strips are convected into the flow and integrated to form vortex particles. As such, the vortex particles will be created in a time marching manner as the near-wake strips evolve.

\subsection{The ONERA Dynamic Stall Model}

Dynamic stall occurs on airfoils, wings, or rotor blades when they are exposed to an unsteady pitching or plunging motion during which the static stall angle of the airfoil is exceeded. The physics that drive the dynamic stall process have been well documented in literature [45], but will be briefly described here.

As the angle of attack of a blade is increased during a pitching motion beyond 


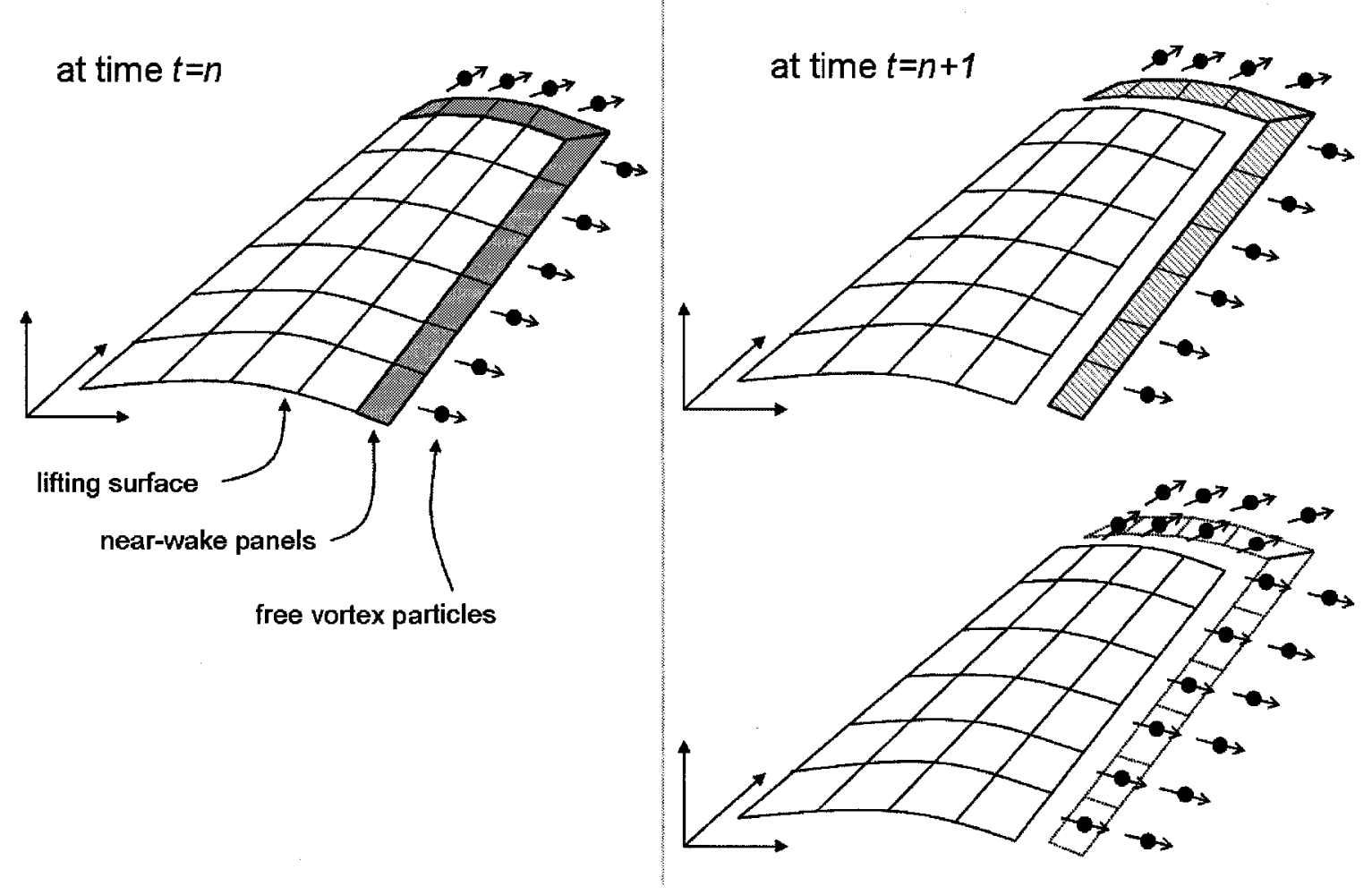

Figure 6.1: The coupling between the near and far wakes.

the airfoil's static stall angle, some boundary layer separation will occur, but the lift created by the airfoil will continue to increase. The flow will begin to separate from the leading edge of the blade and will create a vortex near the leading edge. As the angle of attack continues to increase, the vortex will convect with the fluid over the upper surface of the blade, increasing the lift. As the vortex reaches the trailing edge of the blade, it is convected into the wake and the flow over the airfoil rapidly becomes fully separated, resulting in a large loss of lift and a strong nose down pitching moment. Once the angle of attack is low enough (typically much lower than the static stall angle), the flow will reattach to the upper surface from the leading edge to the trailing edge. The effect of dynamic stall on the lift behaviour of an airfoil is described in Figure 6.2

Dynamic stall can occur on wind turbine blades due to rapid changes in the wind 


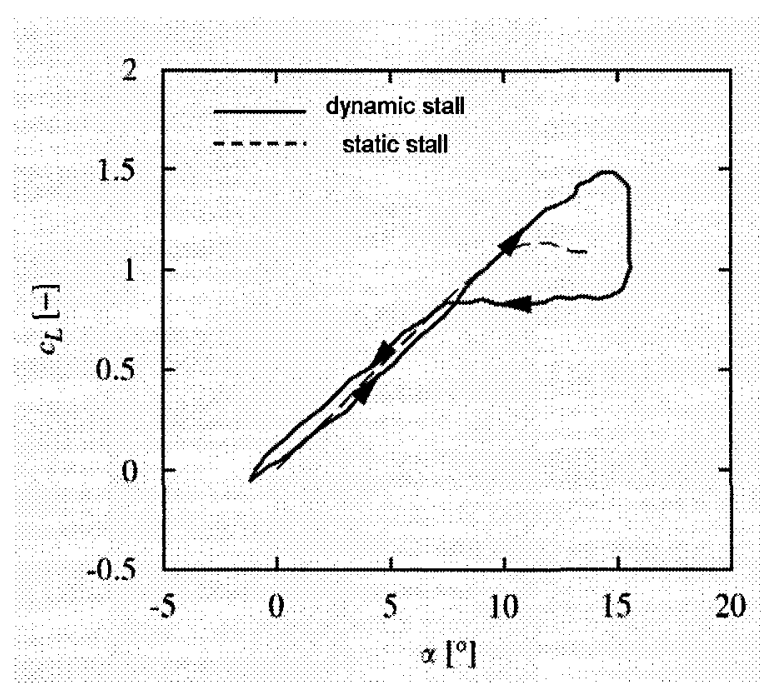

Figure 6.2: Effect of dynamic stall on the lift coefficient. Reproduced from [106].

speed, or due to changes in the wind direction, leading to yawed flow situations where the local angle of attack will change with rotor blade azimuth angle. Though Navier-Stokes CFD solvers can be used to predict and analyze the effects of dynamic stall, dynamic stall models are typically used in research or commercial software [45]. Several common stall models are catalogued in [45] and include the ONERA model [107], the Boeing-Vertol model [108], and the Beddoes-Leishman model [109].

The ONERA model was originally implemented in GENUVP [110] and has been demonstrated by several authors to adequately capture the lift, moment, and drag characteristics during dynamic stall on a variety of airfoils $[111,106]$. The ONERA model was originally developed by Tran and Petot in 1981 [107] at the Office Nationale d'Etudes et de Recherches Aeronautiques (ONERA) in France. The model was developed on the basis of using differential equations to determine the aerodynamic coefficients for each blade section element during a pitching motion. The original model was extended by Peters in 1985 [112] to include plunge motions and has been expressed using either the aerodynamic coefficients, or by the normalized circulation per unit length $[113,114]$. 
A detailed description of the derivation of the model and the wind tunnel tests required to characterize and identify the model coefficients can be found in [115]. However, an excellent summary of the original ONERA model in aerodynamic coefficient form, the extended ONERA model in circulation form, and the suggested coefficients that can be used to implement the model for a mean airfoil is given by Petot [111]. A summary of the general differential equations used to model the lift, moment, and drag coefficients in the ONERA model will be given in this section, and the discretization, numerical implementation, and the list of the coefficients applied in SMARTROTOR is presented in Appendix C.

The original version of the ONERA dynamic stall model was originally implemented in SMARTROTOR as developed for pitching motions, though it can be used for more general motions. An aerodynamic coefficient $C$ is expressed as the sum of two terms in the ONERA dynamic stall model; one term $C_{1}$ to account for the attached component of the flow and a second term $C_{2}$ to account for the separated flow, as seen in Equation C.1.

$$
C=C_{1}+C_{2}
$$

$C_{1}$ represents the load contribution from attached flow and is represented by a first order differential equation for the lift coefficient as seen in Equation 6.22 and by an explicit expression for the drag or moment coefficient as expressed in Equation 6.23. The parameter $C_{l i n}$ represents the linear portion of the lift, moment, or drag curve in the absence of stall.

$$
\begin{gathered}
\dot{C}_{1}+\lambda C_{1}=\lambda C_{l i n}+(\lambda s+\sigma) \dot{\alpha}+s \ddot{\alpha} \\
C_{1}=C_{l i n}+(s+\sigma) \dot{\alpha}+s \ddot{\alpha}
\end{gathered}
$$


In Equations 6.22 and 6.23 , the coefficients $\lambda, s$, and $\sigma$ can be identified through wind tunnel testing. The second contribution $\left(C_{2}\right)$ to the overall coefficient $C$ is determined using the second order differential equation seen in Equation 6.24. In Equation 6.24, the variable $\Delta C=C_{\text {lin }}-C_{s}$ represents the difference between the linear aerodynamic coefficient and the actual aerodynamic coefficient in stall $C_{s}$, as determined from a static lift, drag, or moment curve.

$$
\ddot{C}_{2}+a \dot{C}_{2}+r C_{2}=-[r \Delta C+E \dot{\alpha}]
$$

The parameters $r, a$, and $E$ in equation 6.24 can be determined using Equations 6.25, 6.26, and 6.27. The additional coefficients in Equations 6.25, 6.26, and 6.27, along with all of the other model coefficients for attached and separated flow, should technically be derived from wind tunnel experiments for the particular airfoil in question. However, Petot has noted that the coefficients are approximately independent of the selected airfoil, and has suggested coefficients for a mean airfoil [111]. As exact coefficients have only been determined for a few airfoils, it is common practice to use the mean airfoil coefficients determined for the ONERA model. The selected dynamic stall coefficients and the numerical implementation of the ONERA model in SMARTROTOR can be found in Appendix C.

$$
\begin{gathered}
\sqrt{r}=r_{0}+r_{2} \Delta C^{2} \\
a=a_{0}+a_{2} \Delta C^{2} \\
E=E_{2} \Delta C^{2}
\end{gathered}
$$

It should be noted that the ONERA dynamic stall model was developed using 
two-dimensional oscillating airfoils but is typically applied to three-dimensional flows. Dynamic stall will be a three-dimensional effect whenever a rotor is present, whether studying a helicopter or wind turbine. Results published in [45] demonstrate that two-dimensional models applied at successive radial strips along a blade can produce engineering level agreement with three-dimensional measurements. It is noted that the tip vortex appears to override the significant onset of dynamic stall near the blade tip however, a phenomenon that may not be properly characterized by existing models.

\subsection{Rotor Blade Structural Modeling}

Helicopter rotor blades are typically modeled with a rotating beam approximation based on linear deformation theory. However, helicopter rotor blades experience large deformations and invalidate the assumptions inherent in the equations of motion based on linear deformation theory. The inertial and elastic coupling between the degrees of freedom of the blade due to the mass and structural properties distributions should also be considered in the structural analysis of helicopter rotors [3]. Small wind turbine rotors and particularly stall-controlled rotors are very stiff and have conventionally been modeled using linear beam theory [47]. However, large commercial wind turbines with diameters of up to $120 \mathrm{~m}$ will experience large structural deformations during their operation, which will be compounded by wind gusts, large inertial loads and yaw misalignment.

The structural model that was implemented into SMARTROTOR by Opoku [3] was designed to accurately model helicopter blades, which are very flexible but has been applied to wind turbine rotor blades in this work. A complete description of the theory of the structural dynamics model can be found in [3] but a brief overview of the model and the aeroelastic coupling method will be discussed here for completeness. 


\subsubsection{Structural Model Theory and Implementation}

The structural model implemented in SMARTROTOR is based on the work of Hodges [116] and Shang [117]. A geometrically exact representation of the dynamics of a rotating beam was developed by Hodges [116]. The formulation used by Hodges decomposes the analysis of a three-dimensional blade structure into a linear analysis of the cross-sectional geometry and material properties and a non-linear one-dimensional analysis along the beam reference line. This geometrically exact formulation is suitable for modeling the large deformations that helicopter rotors and large wind turbine rotors will experience in their operational environment. Additionally, Hodges' representation allows inertial and elastic coupling between the beam degrees of freedom to be modeled [3].

Hodges' work was extended by Shang who formulated Hodges' mixed variational equations of motion in a global rotating frame and applied a finite element method to the equations. The structural code implemented into SMARTROTOR was created by Cheng [118] based on Shang's formulation, including a modification to account for the effects of integral twist actuation $[86,119]$

The spatial domain of the blade is discretized into $N$ elements and a set of partial differential equations can be written and are expressed in matrix notation in Equation 6.28 .

$$
F_{S}(X, \dot{X})-F_{L}=0
$$

In Equation 6.28, $F_{S}$ is the structural matrix operator that contains the complete nonlinear equations of motion and is presented in [3], $F_{L}$ is the load operator, and $X$ is the unknown vector consisting of structural variables. The components of the unknown variables in $X$ depend on the boundary condition of the hingeless rotor 
blade, and can be seen in Equation 6.29 [86]

$$
X=\left[\hat{F}_{1}^{T} \hat{M}_{1}^{T} u_{1}^{T} \theta_{1}^{T} F_{1}^{T} M_{1}^{T} P_{1}^{T} H_{1}^{T} \ldots u_{N}^{T} \theta_{N}^{T} F_{N}^{T} M_{N}^{T} P_{N}^{T} H_{N}^{T} \hat{u}_{N+1}^{T} \hat{\theta}_{N+1}^{T}\right]^{T}
$$

The unknown variables in $X$ include the nodal forces $(F)$, moments $(M)$, displacements $(u)$, and rotations $(\theta)$, along with the elemental linear momentum $(P)$ and angular momentum $(H)$ quantities. The $\left({ }^{\wedge}\right)$ term refers to boundary values and the subscripts in Equation 6.29 refer to the node number. For a hingeless rotor blade, $\hat{u}_{1}=\hat{\theta}_{1}=\hat{F}_{N+1}=\hat{M}_{N+1}=0$.

The derivative terms in Equation 6.28 are integrated in time using a second-order backward Euler method [86]. The integrated quantities can be used to obtain the set of nonlinear algebraic equations at a time step $n$ described by Equation 6.30.

$$
F_{S}\left(X^{n}\right)-F_{L}=0
$$

The nonlinear equations described by Equation 6.30 are solved iteratively using the Newton-Raphson method [120] and the result describes the displacement, stress and strain fields of the beam at each time step. The reader is directed to the works by Opoku [3] and [118] for a more detailed discussion of the structural component theory.

\subsubsection{Aeroelastic Coupling with GENUVP}

SMARTROTOR is a closely coupled code, and the rotor blades are modeled separately by the structural and aerodynamic components. As mentioned in the previous section, the structural component uses a one-dimensional beam element discretization following the beam reference axis. The aerodynamic component, GENUVP, models each lifting surface using a discretized surface composed of panels. At each spanwise segment of the lifting surface, the section loading and elasticity are provided by the 
aerodynamic and structural components, respectively [3]. The aerodynamic component solves for the potential and viscous loading at each spanwise strip. The spanwise loading distribution is then transferred by the structural solver to the beam reference axis and the aeroelastic response of the beam is determined. The elastic deformation determined by the structural solver is then used to deform the geometry of the panels on the lifting surface. The plunge, lag, extension, and pitch degrees of freedom are used for the aeroelastic coupling. The rate of elastic deformation is also determined and results in an additional lifting surface velocity component $F$ that is accounted for in the non-penetration boundary condition used by GENUVP (see Equations 6.5 and 6.11).

The aerodynamic and elastic loads and deformations are exchanged at each time step in SMARTROTOR using an interface routine described by Opoku [3]. The meshes used for the aerodynamic and structural meshes are coincident in the spanwise direction of each lifting surface. As such, the width of each structural beam element is the same as the width of the corresponding spanwise strip of aerodynamic panels. Additionally, the structural and aerodynamic solvers use the same time step during simulations. The inputs to the structural model at each spanwise beam element are the inverse mass and stiffness matrices, where the mass and stiffness matrices are given by Equations 6.31 and 6.32. The terms $m_{1}, m_{2}$, and $m_{3}$ in Equation 6.31 refer to the directional mass distribution within an element and $I_{11}, I_{22}$, and $I_{33}$ are the mass moments of inertia about the $\mathrm{x}, \mathrm{y}$ and $\mathrm{z}$ axes. In the stiffness matrix, $E A$ is the axial stiffness of the blade, $G J$ is the torsional stiffness, and $E I_{F L A P}$ and $E I_{L A G}$ represent the flapwise and edgewise stiffness terms, respectively. 


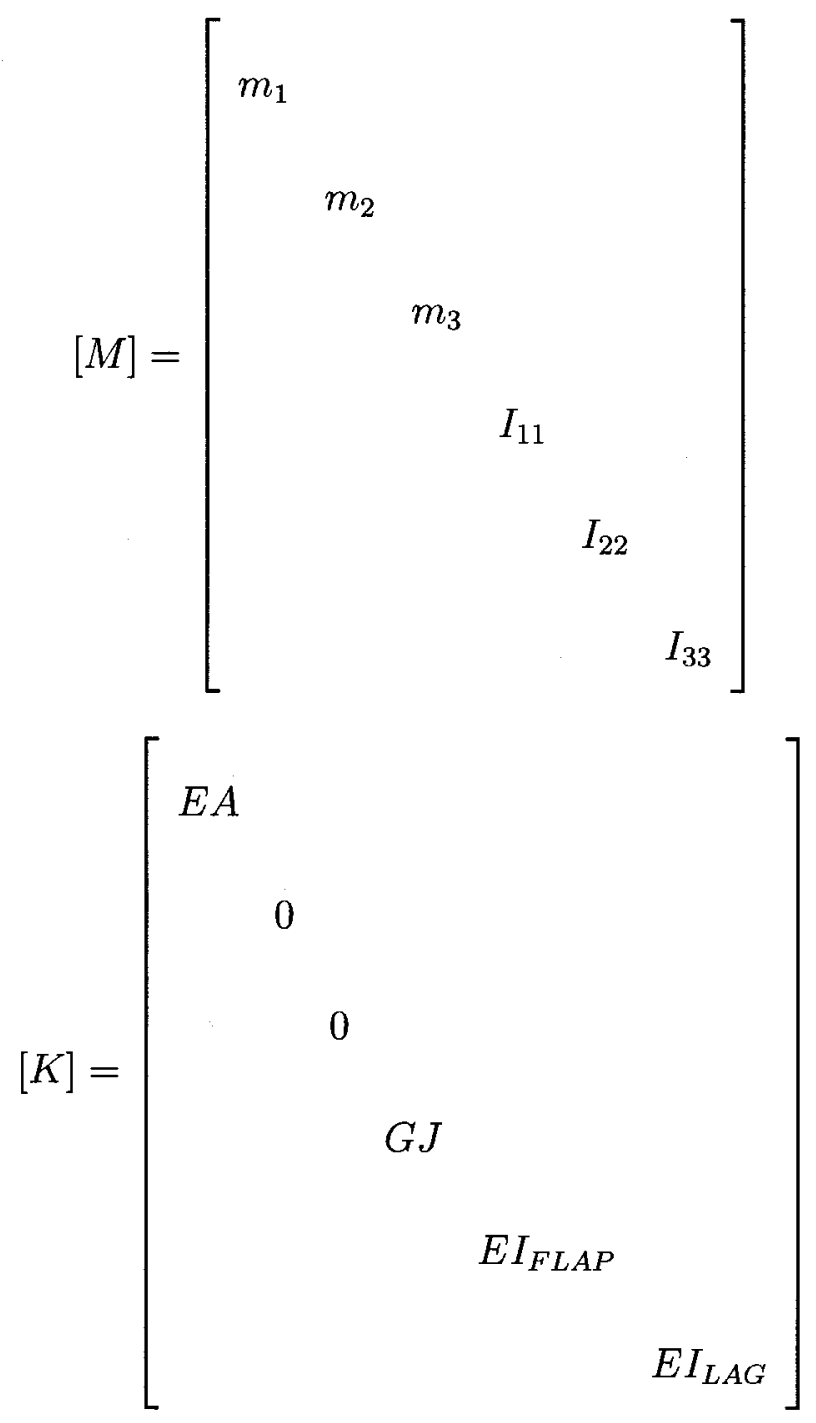




\section{Chapter 7}

\section{Results}

SMARTROTOR was validated as an industrial tool for wind turbine performance prediction using the National Renewable Energy Laboratory (NREL) $10 \mathrm{~m}$ turbine experiment in a series of simulations.

\subsection{Rigid Body Aerodynamic Results}

The NREL S809 airfoil was selected for validation as the airfoil has been implemented on several wind turbine rotors including the NREL experimental turbine. The S809 airfoil was specifically tailored to the requirements of the wind turbine industry and features a low maximum lift coefficient and gentle stall characteristics. The design, development, and initial testing of the S809 airfoil is documented in [121] and steady and unsteady wind tunnel testing of the airfoil can be found in [122]. A crosssection of the airfoil can be seen in Figure 7.1, in which the mean camber line is represented with a dashed line. The current version of SMARTROTOR uses a thin airfoil approximation, where the airfoil is represented by panels mapped onto its mean camber line. 


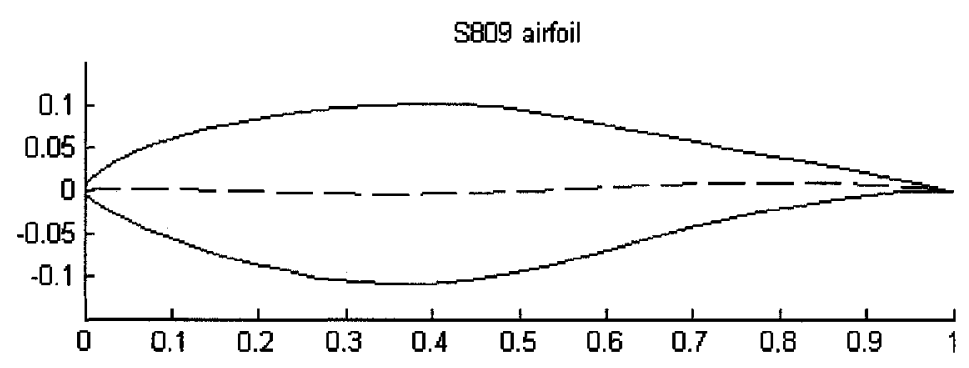

Figure 7.1: Profile of the S809 Airfoil [122].

\subsubsection{S809 Static Lift Curve}

A static lift curve for the S809 airfoil was created using SMARTROTOR in order to validate the code's prediction of the effective angle of attack under static loads and to validate the thin wing representation of the S809 airfoil, which is a $21 \%$ thick section. The test conditions used to develop the static lift curve can be seen in Table 7.1. A high aspect ratio wing was used to limit the three-dimensional effects characteristic of SMARTROTOR and to approximate the two-dimensional conditions of the experimental test cases. The experiment performed at the Ohio State University (OSU) was performed at Reynolds numbers from $0.75 \times 10^{6}$ to $1.5 \times 10^{6}$ in conditions representative of wind turbine rotor operating environments. The static lift curve has only been compared to the experimental results obtained at a Reynolds number of $1.0 \times 10^{6}$, but it should be noted that the airfoil did not demonstrate high Reynolds number sensitivity in the experiment.

The lift curve for the S809 airfoil produced with SMARTROTOR is compared to the experimental results from the Ohio State University [122] in Figure 7.2. As shown in Figure 7.2, the results of the simulation closely match the experiment with some deviations at large positive and negative angles of attack. SMARTROTOR uses potential flow aerodynamics to determine the effective angle of attack of an airfoil section, and then accounts for stall by using a lookup table for the lift and drag coefficients. The pitching moment coefficient of the section is also obtained from the 
lookup table to reduce the need for panel refinement near the leading edge of a strip. The results of Figure 7.2 demonstrate that the code properly predicts the effective angle of attack under steady two-dimensional loading conditions, and that the thin wing assumption is valid despite the fact that the S809 airfoil has a $21 \%$ thickness to chord ratio.

Table 7.1: S809 static lift curve test conditions

\begin{tabular}{|c|c|}
\hline Span & $17 \mathrm{~m}$ \\
\hline Chord & $1 \mathrm{~m}$ \\
\hline Panel density & 16 spanwise $\times 8$ chordwise \\
\hline Airfoil type & S 809 \\
\hline Reynolds number & $1.01 \times 10^{6}$ \\
\hline Kinematic viscosity & $1.56 \times 10^{-5} \mathrm{~m}^{2} / \mathrm{s}$ \\
\hline Inflow velocity & $15.5 \mathrm{~m} / \mathrm{s}$ \\
\hline Angle of attack range & $-20^{\circ}$ to $26^{\circ}$ \\
\hline
\end{tabular}

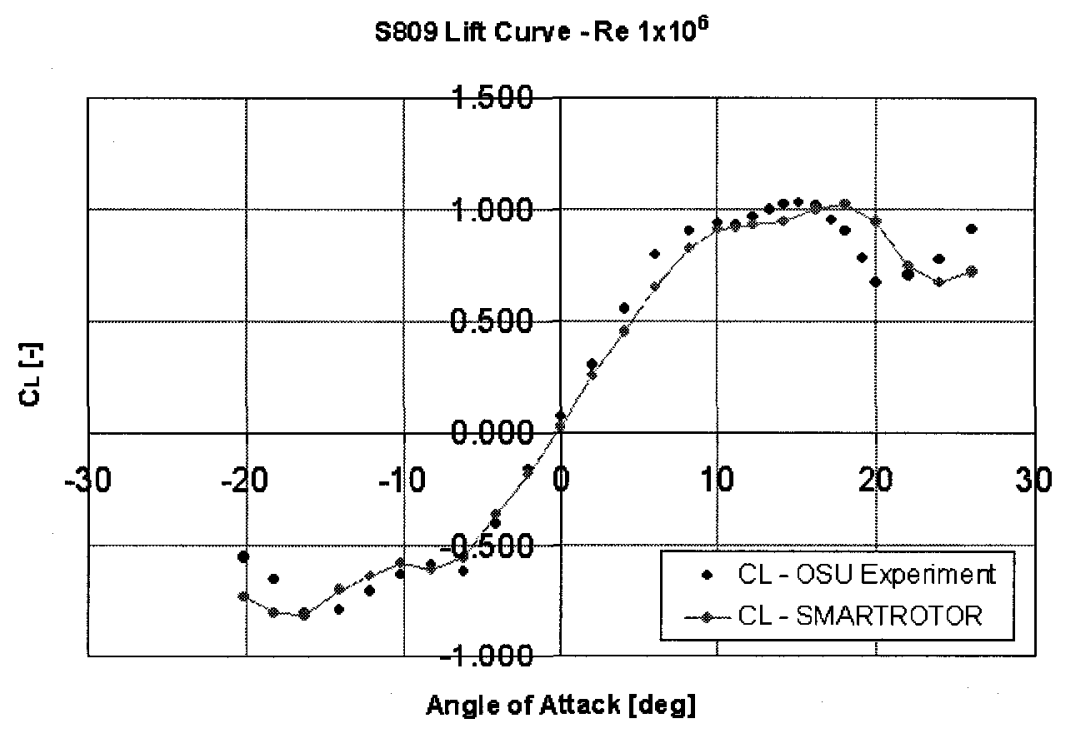

Figure 7.2: Static lift curve for the S809 airfoil. 


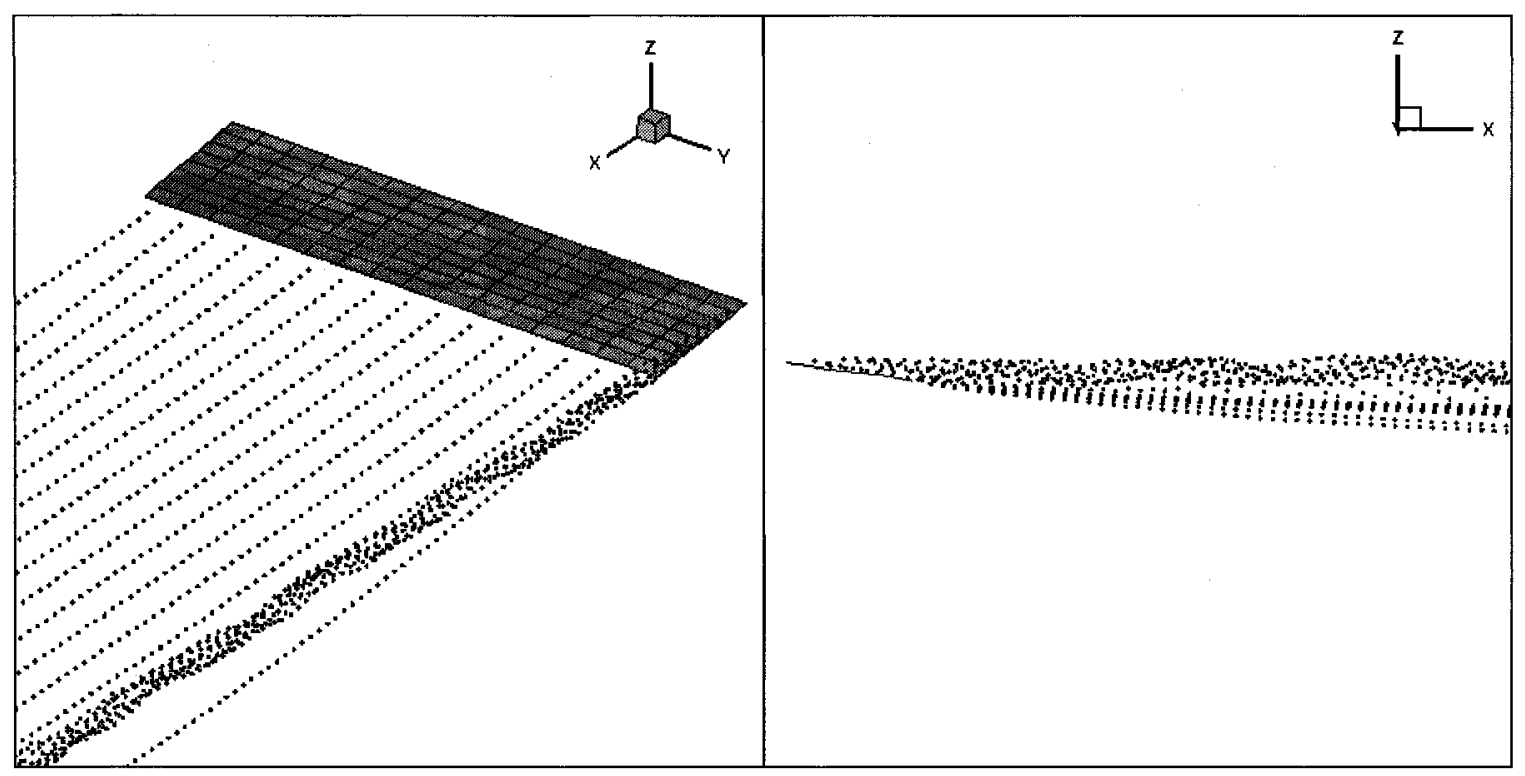

Figure 7.3: Visualization of the wake with an $8^{\circ}$ angle of attack.

The rectangular wing used to perform the steady lift coefficient simulations can be seen in Figure 7.3 with an $8^{\circ}$ angle of attack. Vortex particles are emitted from the trailing edge of the wing at each spanwise strip and from each chordwise panel at the tip of the wing. The formation of the tip vortex can be seen in the left panel of Figure 7.3 (in which the scale of the y-axis has been modified for visualization) and the behaviour of the induced wake behind the wing can be seen in the right panel of Figure 7.3. The lift coefficient used in the lift curve comparison was determined by taking the average lift coefficient of the middle two strips of the wing.

\subsubsection{S809 Unsteady Simulations}

Wind turbines will experience asymmetric loading in many wind conditions which can induce dynamic stall on the rotor blades. These conditions can be caused by yaw misalignment with the wind, sudden gusts, or by an incident wake from an upstream turbine. As such, it was desired to validate the unsteady load prediction capabilities of SMARTROTOR using the ONERA dynamic stall model described in Section 6.2. The 
Table 7.2: S809 unsteady lift curve test conditions

\begin{tabular}{|c|c|}
\hline Span & $17 \mathrm{~m}$ \\
\hline Chord & $1 \mathrm{~m}$ \\
\hline Panel density & 16 spanwise $\mathrm{x} 8$ chordwise \\
\hline Airfoil type & $\mathrm{S} 809$ \\
\hline Reduced frequency & 0.077 \\
\hline Reynolds number & $1.01 \times 10^{6}$ \\
\hline Kinematic viscosity & $1.56 \times 10^{-5} \mathrm{~m}^{2} / \mathrm{s}$ \\
\hline Inflow velocity & $15.5 \mathrm{~m} / \mathrm{s}$ \\
\hline Pitch oscillations & $8 \pm 5.5^{\circ}$ \\
& $14 \pm 5.5^{\circ}$ \\
\hline
\end{tabular}

rectangular wing used for the steady simulations was used for the unsteady test cases. Similar to the steady simulations, the selected test conditions were set based on the experiments performed at the Ohio State University which were specifically tailored to the unsteady operational conditions of wind turbines. Unsteady pitch oscillations were performed over the angles $8 \pm 5.5^{\circ}$ and $14 \pm 5.5^{\circ}$ with a reduced frequency (k) of 0.077 according to Equations 7.1 and 7.2. The remaining test conditions can be seen in Table 7.2. As the unsteady wind tunnel experiment performed by the OSU [122] only documented the Reynolds number used for each test, the velocity $\left(U_{\infty}\right)$ and kinematic viscosity $(\gamma)$ used in the SMARTROTOR simulations had to be estimated from the experimental test report. The unsteady pitching motions used a resolution of 50 steps per cycle and lift data from the second cycle with a wake was used.

$$
\begin{gathered}
\alpha=\alpha_{\text {mean }} \pm \Delta \alpha(\sin \omega t) \\
\omega=\frac{2 k U_{\infty}}{c}
\end{gathered}
$$


An initial simulation was conducted without the ONERA dynamic stall model to observe how SMARTROTOR predicted the stall hysteresis using only inviscid potential forces. As expected, the unsteady lift curve shown in Figure 7.4 (with a dashed line) demonstrates that SMARTROTOR does a poor job of representing the unsteady behaviour characteristic of dynamic stall without the ONERA model.

The ONERA dynamic stall model has 9 coefficients that describe the attached flow and separated flow behaviour of the differential equations for lift as discussed in Section 6.2. The acceptable range for each coefficient in the ONERA dynamic stall model is presented by Petot in [111] along with coefficients that can be used for a mean airfoil shape. The mean ONERA coefficients were used to observe how well the behaviour of the $\mathrm{S} 809$ airfoil in dynamic stall was predicted. As seen in Figure 7.4, the original ONERA dynamic stall model poorly captures the stall hysteresis observed in the experiment. The ONERA model coefficients were originally designed for helicopter rotors operating conditions and may not be ideally tailored to wind turbine blade conditions. It was noted in [106] that the coefficients of the BeddoesLeishman dynamic stall model had to be modified in order to properly characterize the behaviour of the S809 airfoil when trying to match the OSU experimental data, and so it was decided to modify two of the ONERA coefficients that describe the attached flow differential equation. The revised coefficients can be found in Appendix $\mathrm{C}$, and the unsteady S809 lift curve produced with the calibrated ONERA model over the angles $8 \pm 5.5^{\circ}$ can be seen in Figure 7.5. The ONERA model with the calibrated coefficients properly characterizes the loading on the wing, but the peak amplitude of the lift coefficient is lower than the experimental results. Some of this error may have occurred as many of the simulation parameters had to be estimated based on the limited information provided in the experimental test report [122]. The dynamic stall results closely match those obtained by Gupta [36]. A second simulation was performed over the interval $14 \pm 5.5^{\circ}$ to observe the behaviour of the calibrated 
Unsteady Lift Curve - $\mathbf{8 8 0 9}$ Airfoll

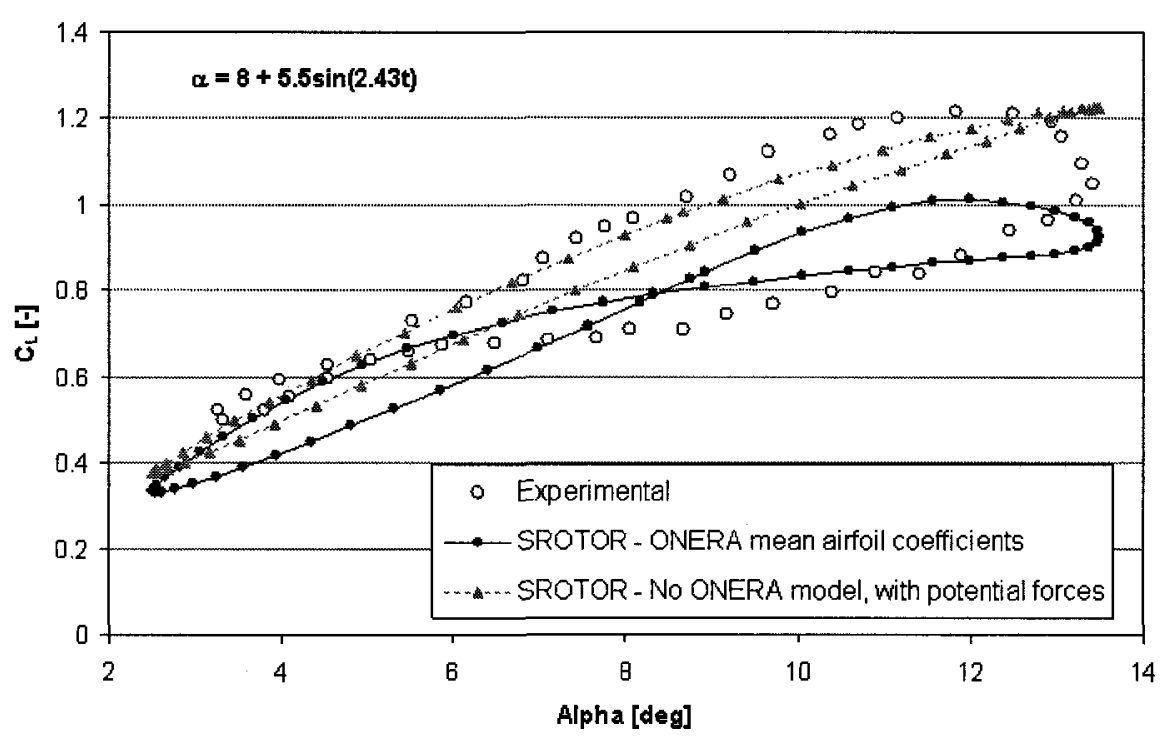

Figure 7.4: Original unsteady lift curve with a reduced frequency of 0.077 .

ONERA model in the fully stalled regime, as presented in Figure 7.6. The model properly captures the increasing lift due to the leading edge vortex development, but fails to capture the flow behaviour during separation and reattachment. The calibrated ONERA dynamic stall model adequately captures the hysteresis present during dynamic stall and will allow SMARTROTOR to perform simulations on turbines in yawed flow, but further calibration of the differential equation coefficients in the fully stalled regime is recommended. 


\section{Unsteady Lift Curve - \$809 Airfoil}

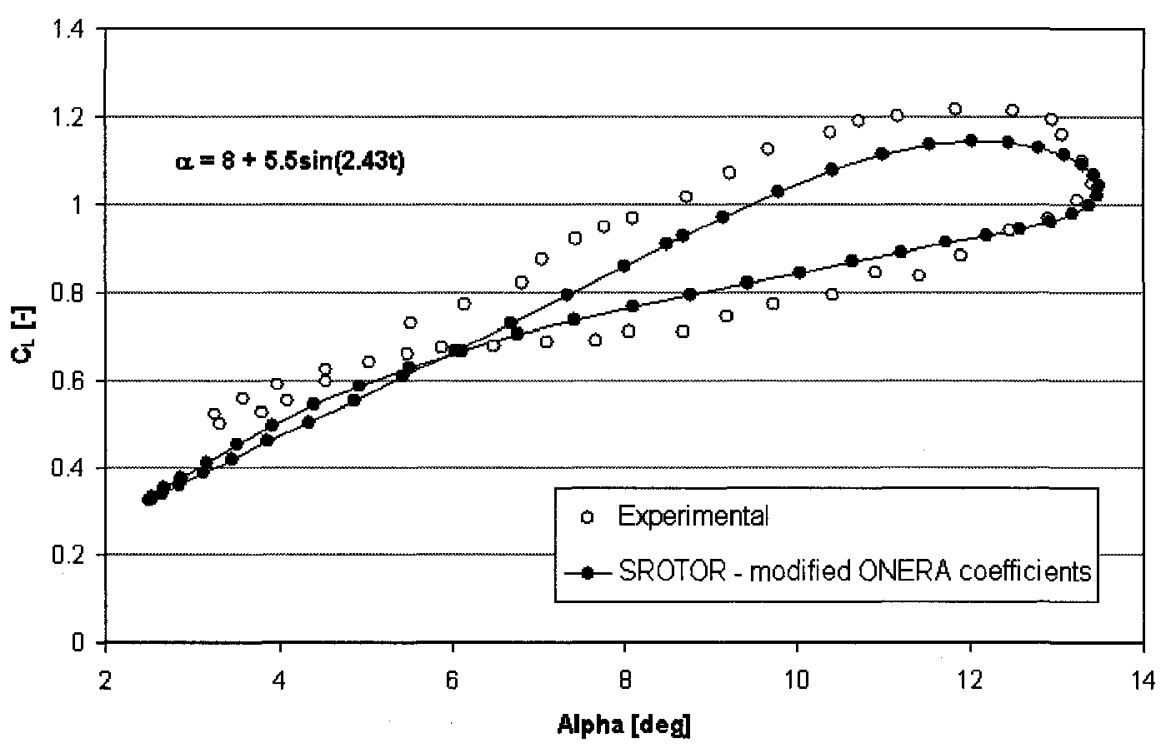

Figure 7.5: Unsteady lift curve with a reduced frequency of 0.077 and an $8^{\circ}$ mean angle of attack.

\section{Unsteady Lift Curve - S809 Airfoil}

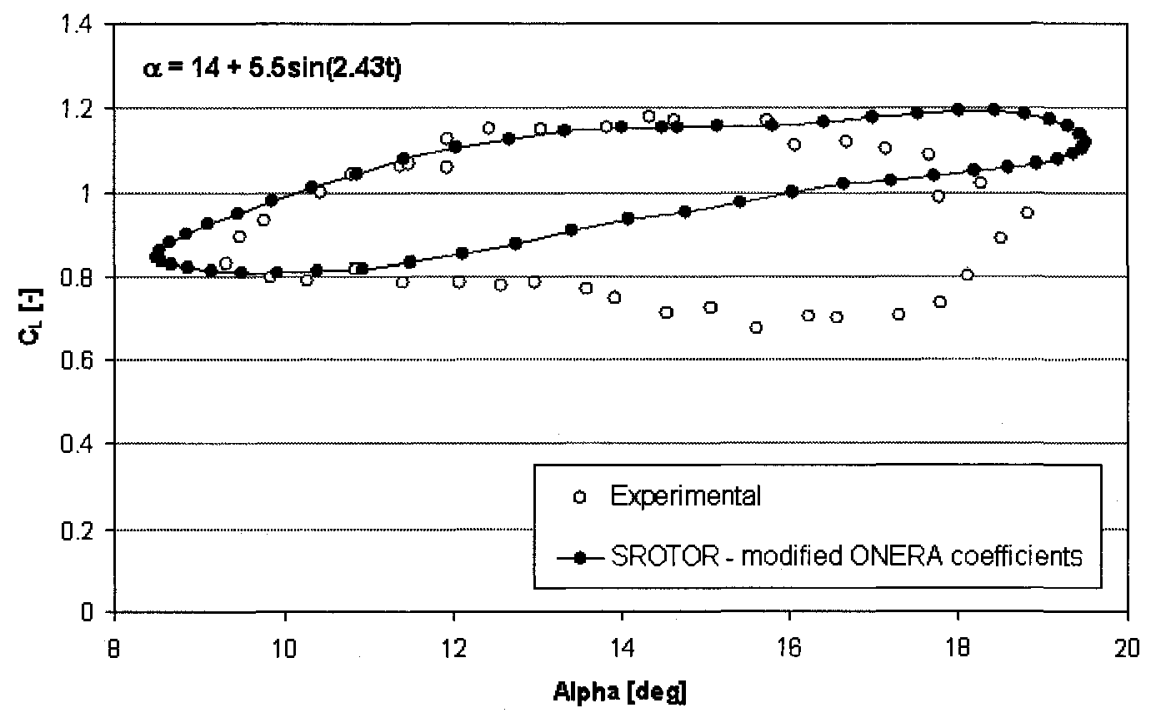

Figure 7.6: Unsteady lift curve with a reduced frequency of 0.077 and a $14^{\circ}$ mean angle of attack. 


\subsection{Aeroelastic Results}

The NREL unsteady aerodynamics experiment (UAE) performed by the NREL was selected as the validation test case for the aeroelastic wind turbine simulations. The NREL turbine has become a benchmark validation case in the wind energy research community since the results of the study were published in 2002 [43], which demonstrated that most currently available commercial and research codes could not accurately predict the torque and power produced by the turbine.

\subsubsection{NREL UAE Phase VI Test Case}

The NREL experiment was conducted in the NASA Ames $24 \mathrm{~m} \times 36 \mathrm{~m}$ wind tunnel using an upwind, two-bladed, twisted, tapered, stall-controlled rotor with a rigid hub and a $0^{\circ}$ cone angle [48]. The NREL Sequence $S$ [97] was selected for the simulations in SMARTROTOR to allow direct comparison with the results of the blind comparison study. The simulation parameters for the selected test case can be seen in Table 7.3.

The experimental NREL blade begins at a radius $r=0.508 \mathrm{~m}$ from the centre of the hub with a cylindrical section until $r=0.883 \mathrm{~m}$. The blade then transitions from a cylindrical cross-section to the S809 airfoil at $r=1.257 \mathrm{~m}$. In order to simplify the blade for SMARTROTOR, the cylindrical and transitional sections were neglected and the root of the blade model was initiated at $r=1.008 m$ with the S809 airfoil. The chord and twist distributions of the NREL blade were interpolated to the 17 spanwise panel nodes, as seen in Figure 7.7, but the blade thickness to chord ratio of $21 \%$ was neglected due to the thin wing representation of the airfoil. The twist distribution indicated in Figure 7.7 is positive towards the feathered blade position, and the tip pitch angle was set to $3^{\circ}$ for the simulations.

The structural properties of the NREL blade were provided in [97] and were 
Table 7.3: NREL turbine simulation parameters.

\begin{tabular}{|c|c|}
\hline Rotor diameter & $10 \mathrm{~m}$ \\
\hline Blade span & $5.029 \mathrm{~m}$ \\
\hline Blade root cutout & $1.008 \mathrm{~m}$ \\
\hline Panel density & 16 spanwise $\times 8$ chordwise \\
\hline Airfoil type & $\mathrm{S} 809$ \\
\hline Rotor speed & $72 \mathrm{rpm}$ \\
\hline Tip pitch angle & $3^{\circ}$ \\
\hline Inflow velocity & 5 to $15 \mathrm{~m} / \mathrm{s}$ \\
\hline Kinematic viscosity & $1.41 \times 10^{-5} \mathrm{~m}^{2} / \mathrm{s}$ \\
\hline Density & $1.225 \mathrm{~kg} / \mathrm{m}^{3}$ \\
\hline Time step & $0.01 \mathrm{~s} / \mathrm{step}$ \\
\hline
\end{tabular}

interpolated to the centre of each of the 16 strips. The mass and stiffness data required at each radial strip in SMARTROTOR includes the principal mass moments of inertia, the directional mass distribution on each element, the axial stiffness, the torsional stiffness, the flapwise and lead-lag stiffness, and the elastic axis of the wing. A table of the structural properties used to model the NREL blade span can be seen in Appendix B. Each blade is assumed to be cantilevered at the root, and does not take into account the flexibility of the blade root. This is a reasonable assumption however, as the NREL structural data indicated that the root of the blade was significantly stiffer than the rest of the blade. It was noticed during the simulations that the blade deflections were less than one centimetre at the tip of the turbine blade in all cases, but increased with increasing wind speed and loading. This is expected due to the short turbine blade radius and due to the high stiffness of stall controlled turbine blades [34]. In a larger commercial turbine however, where the rotor blade radius can increase up to $60 \mathrm{~m}$, it is expected that the tip deflection would be much more pronounced and the benefit of using the nonlinear beam model in SMARTROTOR 


\section{NREL chord and twist distribution}

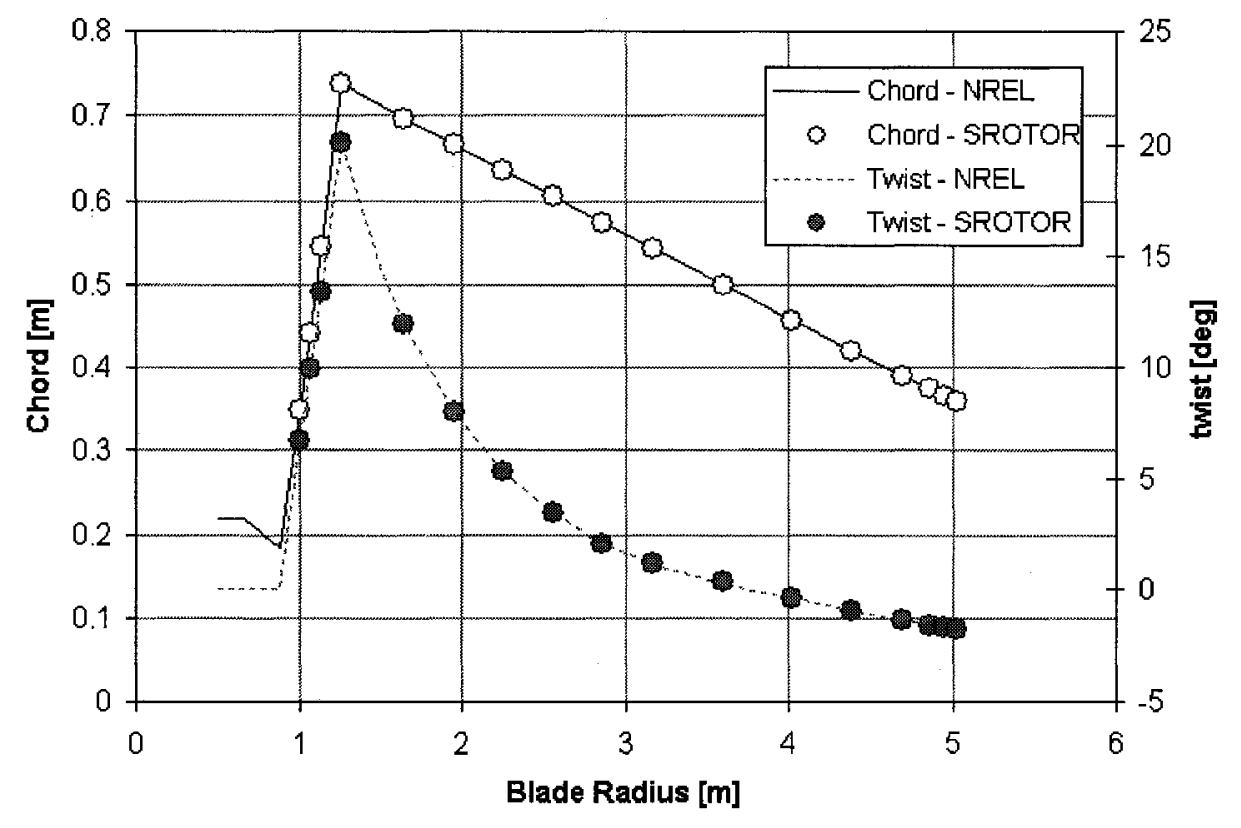

Figure 7.7: Chord and twist distribution for the NREL blade.

would be observed.

The NREL simulations presented in this thesis were performed for 10 rotor revolutions. The air density and the loads are increased linearly over the first 4 revolutions to limit structural oscillations caused by the instantaneous rotor startup following which the simulation continues for 1 revolution with full aerodynamic loading. The particle wake is then activated for the remaining 5 revolutions and the results become periodic after the 3rd revolution of the rotor when the particle wake is active. The convergence behaviour of the torque contribution from one blade throughout ten revolutions can be seen in Figure 7.8. Each simulation using the above procedure with 10 rotor revolutions required between 4 and 5 hours on 1 dual-core $3.2 \mathrm{GHz}$ CPU. It should be noted that, in normal flow situations, the linear increase in density can be shortened and if simulations are stopped once a periodic solution is obtained after three revolutions with the wake, the simulations can be performed in approximately 3 hours on 1 dual-core $3.2 \mathrm{GHz}$ CPU, demonstrating that SMARTROTOR is a very 
rapid simulation tool.

\section{Torque convergence for one blade at $8 \mathrm{~m} / \mathrm{s}$}

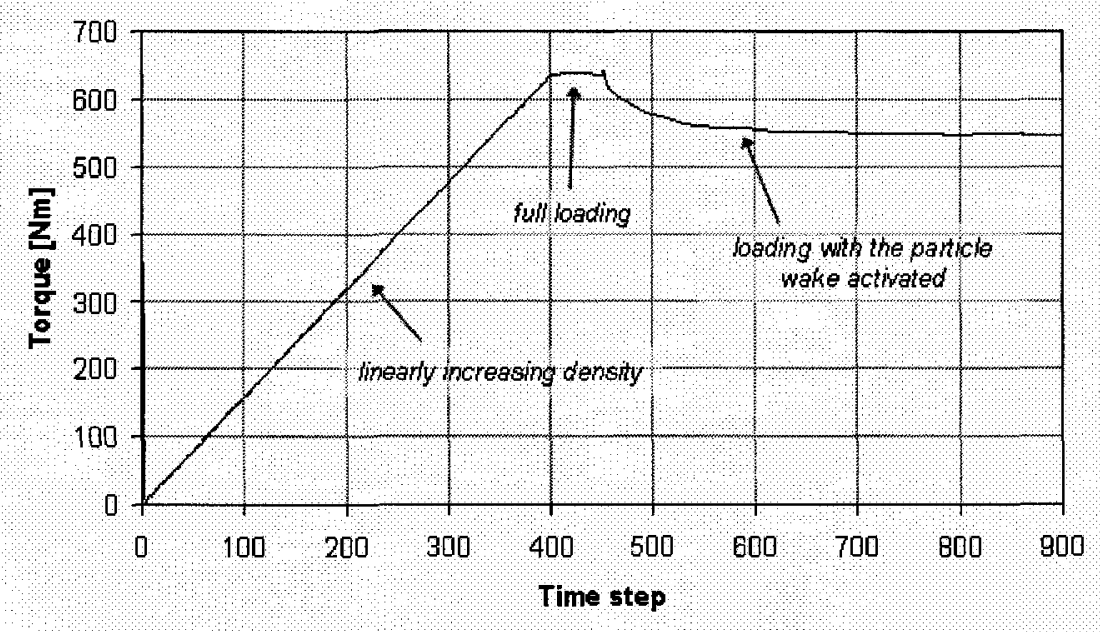

Figure 7.8: Convergence behaviour of the NREL blade torque.

The particle wake after two revolutions can be seen in Figure 7.9, where the particles are shaded according the the y-coordinate for visualization. This Figure demonstrates the importance of modeling the tip and root vortices of a wind turbine and represents the wake in ideal circumstances. If a complete turbine configuration had been modeled, the tip vortex behaviour would be affected once per blade per revolution by the presence of the tower and the root vortex would be affected by the rotor hub and nacelle. The aerodynamic component of SMARTROTOR, GENUVP, can be used to model the tower, nacelle, and hub of a wind turbine using source distributions and will increase the accuracy of simulations in SMARTROTOR. 


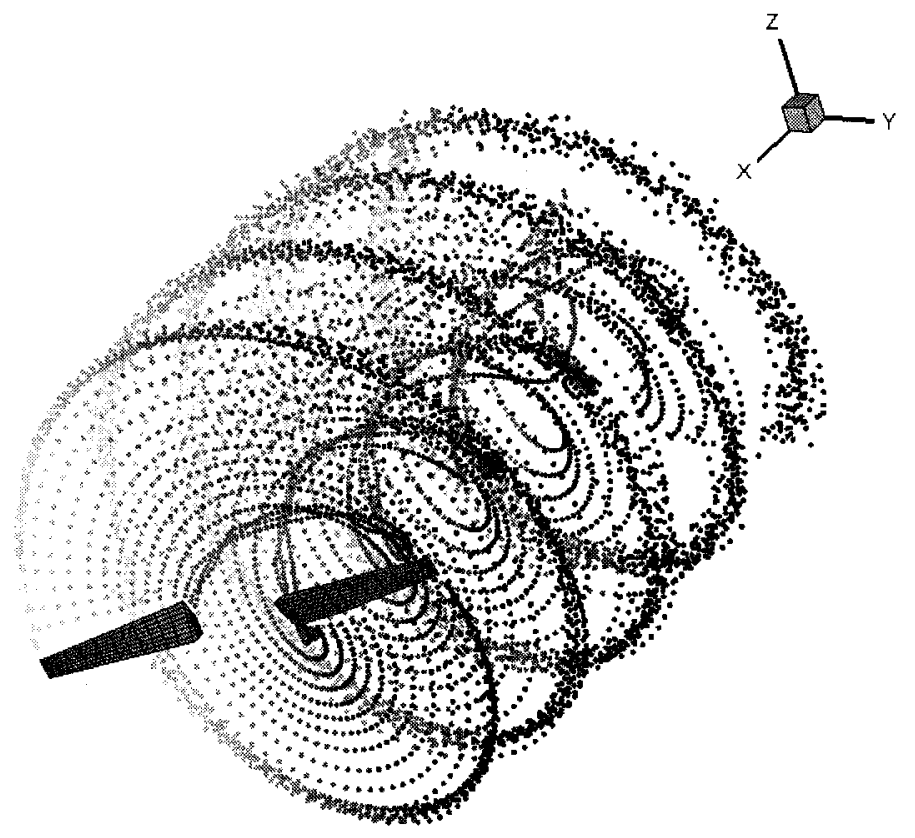

(a) Isometric view.

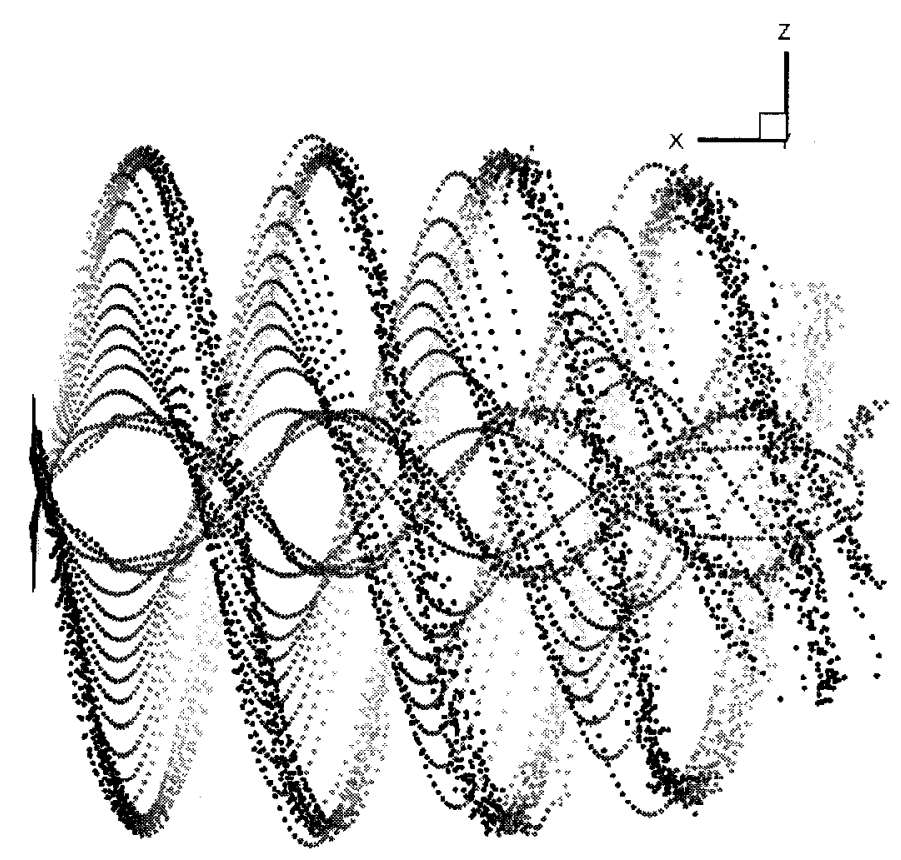

(b) Side view.

Figure 7.9: Visualization of the NREL turbine wake. 
The torque produced by the NREL turbine was calculated in SMARTROTOR and has been superimposed onto the results of the NREL blind comparison study in Figure 7.10. The torque plot was originally published by Leishman [41] and includes results from 8 of the 18 participants in the NREL blind comparison that are representative of the extremes predicted by all of the participants. The best prediction of the NREL power curve was the CFD solution of Sørensen [87] which can be seen in Figure 7.10 as the line that mimics the experimental data points. Participants in the NREL blind comparison study were asked to provide results at $7,10,13,15,20$, and $25 \mathrm{~m} / \mathrm{s}$, but additional experimental results were available in $1 \mathrm{~m} / \mathrm{s}$ intervals from [44] and added to the original plot from [41] for further validation with SMARTROTOR. The predicted power output and the predicted turbine thrust predicted are compared to the NREL experimental results published in [44] in Figures 7.11 and 7.12. As seen in Figures 7.10 and 7.11, the stall-controlled NREL turbine reaches its rated turbine power at approximately $10 \mathrm{~m} / \mathrm{s}$ and the power curve exhibits the wavy behaviour characteristic of stall-controlled turbines above the rated turbine speed. Above the rated wind speed, the majority of the turbine blades will be continuously operating in stalled and deep-stall conditions and the amount of blade stall will govern the output power.

The errors in the torque and power predictions were within $15 \%$ of the experimental data at wind speeds up to and including $10 \mathrm{~m} / \mathrm{s}$, and were overpredicted by $35 \%$ to $40 \%$ at wind speeds higher than $10 \mathrm{~m} / \mathrm{s}$. However, the axial thrust predicted with SMARTROTOR was within $8 \%$ at all simulated wind speeds, indicating that overestimation of the radial loading likely contributed to the error in torque and power. Additionally, overestimation of the effective angle of attack and inadequate characterization of three-dimensional stall effects over the span of the rotor also contributed to the observed errors. Blade stall is a three-dimensional phenomenon on wind turbine rotors and the viscous correction in SMARTROTOR is in effect a two-dimensional 


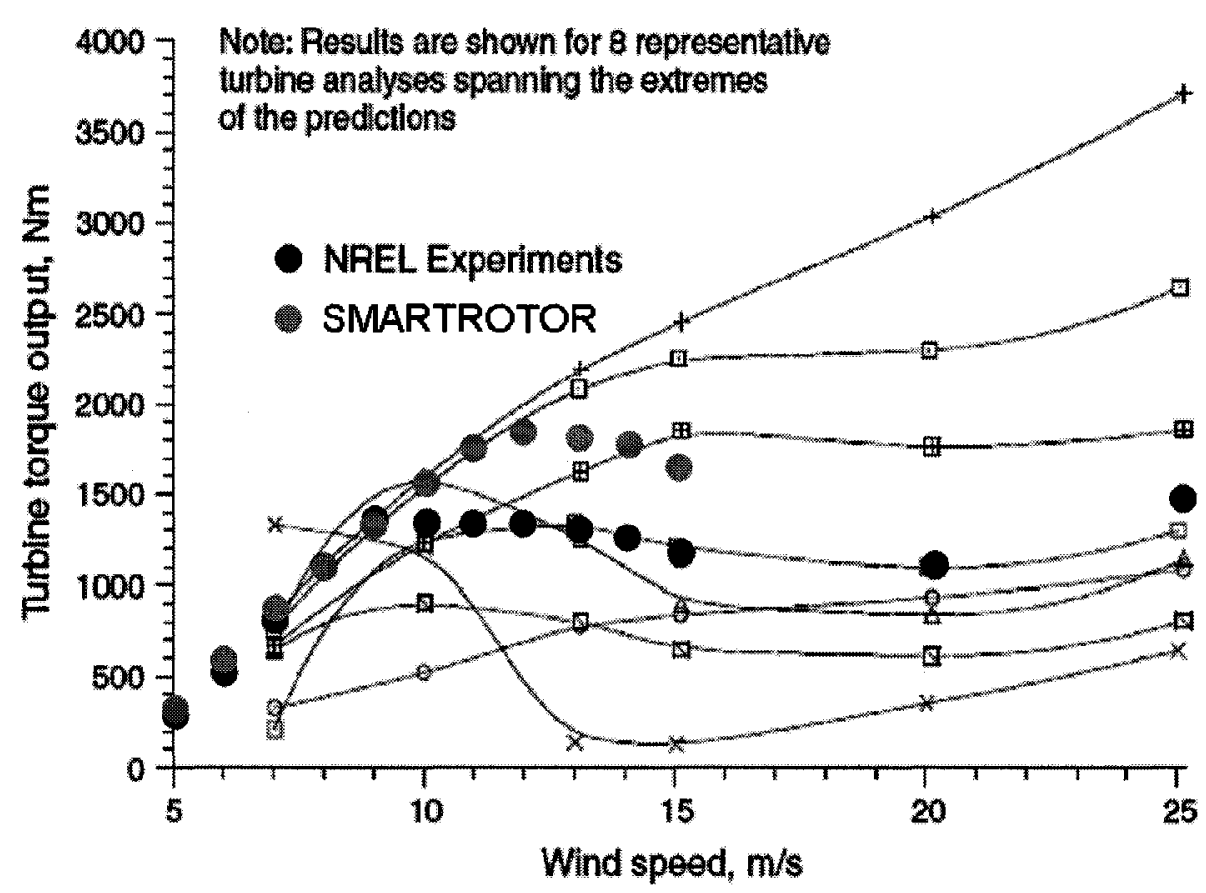

Figure 7.10: Comparison of the NREL turbine torque.

correction with no prediction of leading edge stall. A leading edge separation model (based on the ONERA stall model in circulation form) has been implemented by Riziotis and Voutsinas in GENUVP with mixed results [123], but does not currently exist in SMARTROTOR.

Large commercial wind turbines have variable pitch blades to maintain a low angle of attack on the blades and to eliminate the reliance on stall to control the power output of turbines. SMARTROTOR has demonstrated the ability to accurately predict the power output of a turbine with low blade angles of attack (i.e. at low incident wind speeds in a stall controlled turbine) and at the characteristic mean annual wind speeds previously described in Table 4.1. Additionally, it should be noted that SMARTROTOR has the ability to perform simulations with an unsteady incident wind speed and variable-pitch blade control, a feature that would be very challenging in grid-based CFD. 
Output Power - NREL Turbine

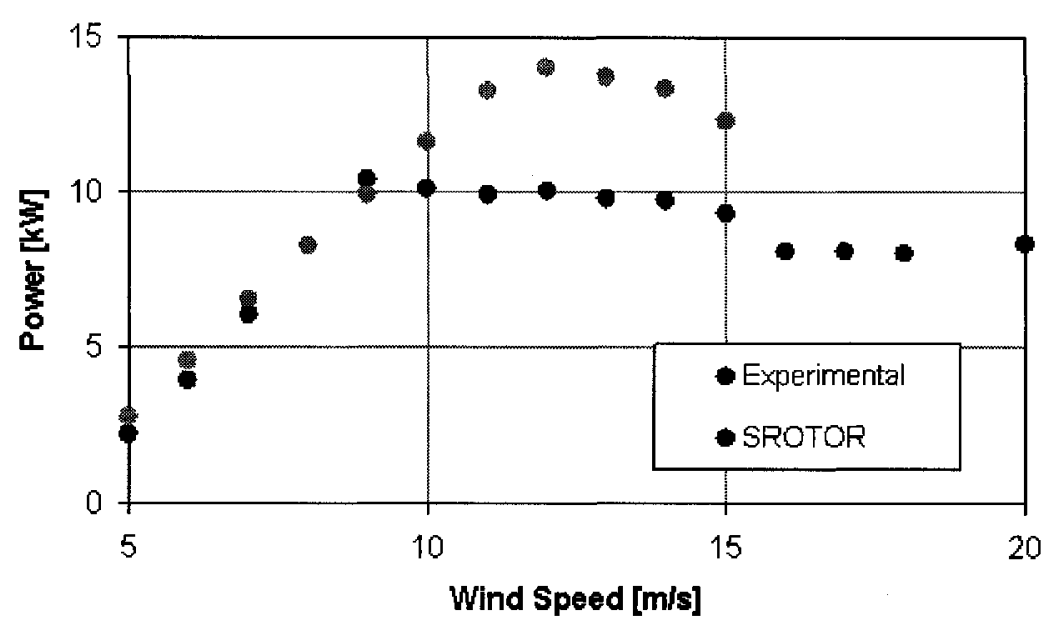

Figure 7.11: Comparison of the predicted NREL turbine power.

\section{Axial Thrust - NREL Turbine}

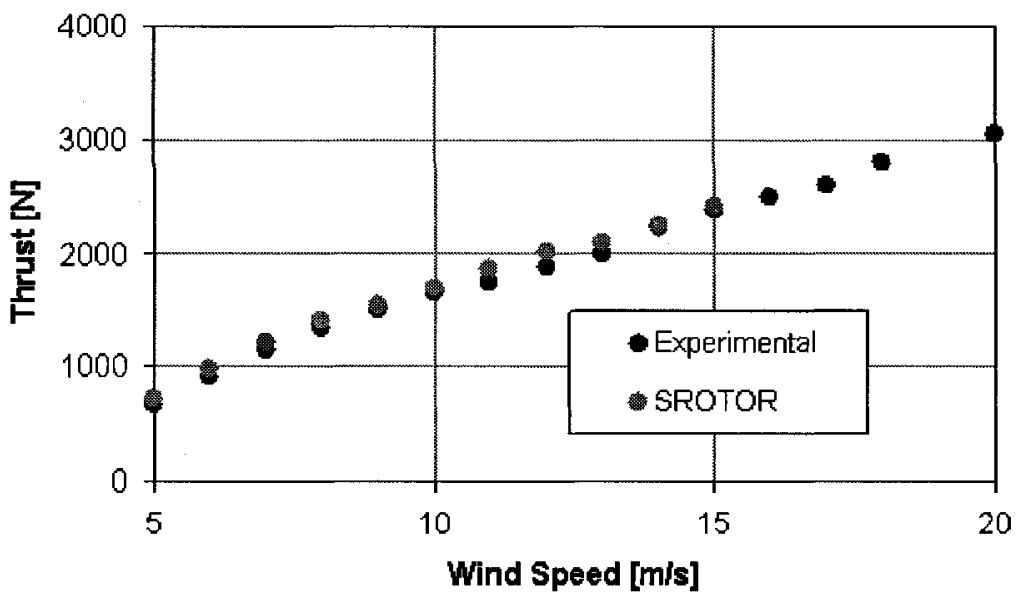

Figure 7.12: Comparison of the predicted NREL turbine thrust. 


\subsubsection{Effects of Yaw Misalignment}

Wind turbines will experience yawed flow conditions due to rapid changes in the incident wind speed and direction. Rotor yaw misalignment and the skewed wake behind the rotor will create unsteady inflow conditions, leading to both unsteady turbine blade loading and unsteady power output. In these conditions, dynamic stall will occur over the span of the rotor blades, further contributing to the unsteady turbine loading. The ONERA dynamic stall model implemented in SMARTROTOR was validated using the experimental results of the NREL UAE Phase VI experiment that were published in [44]. Experimental tests were performed by the NREL where the rotor was yawed $10^{\circ}, 30^{\circ}$ and $60^{\circ}$ to the inflow velocity.

The power and thrust calculated in SMARTROTOR can be seen in Figures 7.13 and 7.14. Experimental results in yawed flow are shown up to $20 \mathrm{~m} / \mathrm{s}$ but yawed flow simulations were performed only at wind speeds where SMARTROTOR accurately predicted the NREL performance in axial flow. The power and thrust shown in Figures 7.13 and 7.14 are overpredicted at each yaw angle. However, the correct trend of decreasing power output with increasing yaw angle was properly determined with SMARTROTOR. There is also a consistent offset between experimental and simulated power output at each wind speed which can be attributed to the calibration of the ONERA dynamic stall model. As demonstrated in the unsteady pitching motion test presented in Figure 7.6, the current implementation of the ONERA model overpredicts the lift coefficient during the vortex shedding and flow reattachment phases in the fully stalled regime. It should be noted that the thrust and power results from SMARTROTOR produced 5 to 10 unstable data points once per revolution that did not affect the mean power or thrust output. These unstable data points were neglected and are attributed to the numerical scheme that had originally been selected for the ONERA dynamic stall model (see Appendix C). 
Output Power of the NREL turbine in Yawed Flow

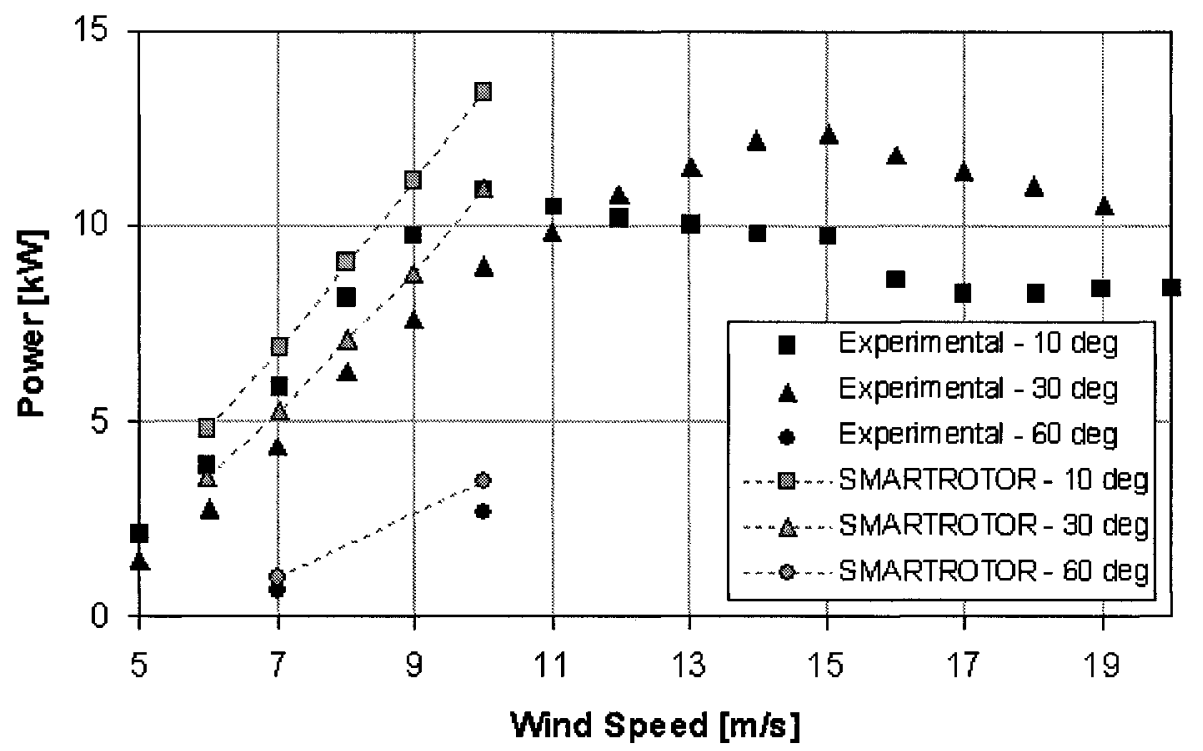

Figure 7.13: Comparison of the predicted NREL turbine power in yawed flow.

\section{Axial Thrust of the NREL turbine in Yawed Flow}

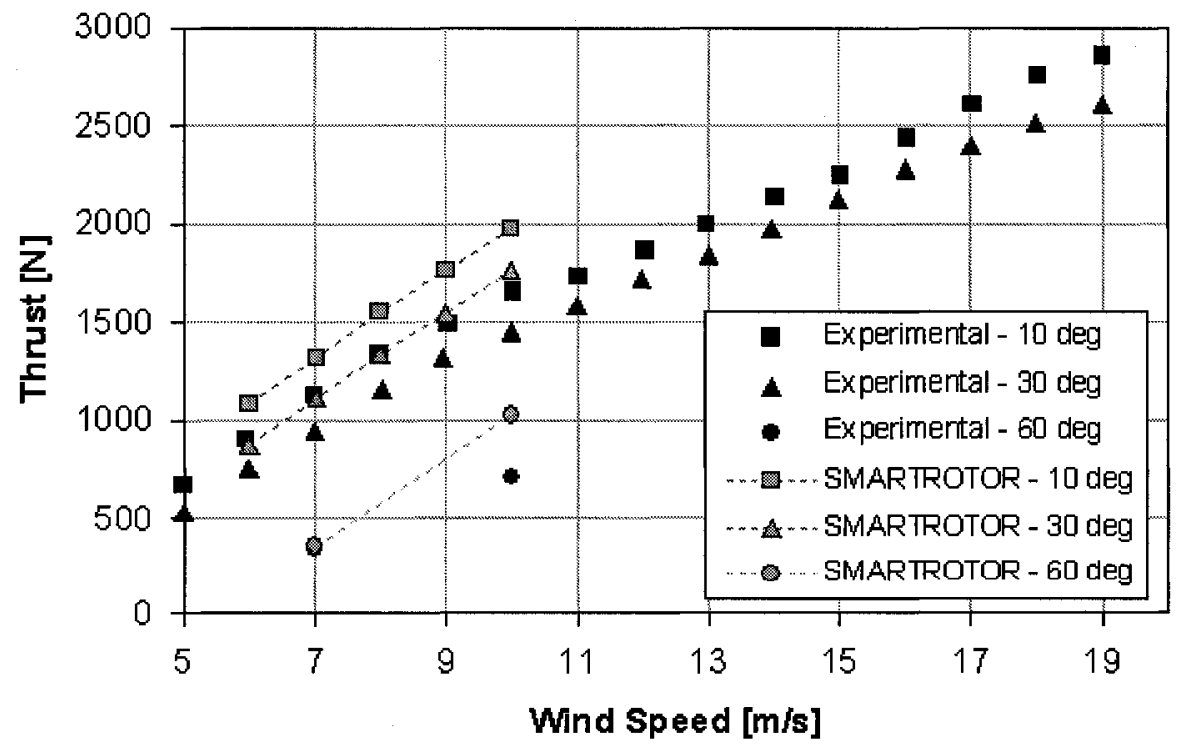

Figure 7.14: Comparison of the predicted NREL turbine thrust in yawed flow. 
The top view of the particles in the wake of the NREL turbine at yaw angles of $10^{\circ}, 30^{\circ}$, and $60^{\circ}$ is shown in Figure 7.15 after four rotor revolutions. The particles in Figure 7.15 are shaded with respect to the vertical z-coordinate for visualization. Though a periodic solution is obtained after three revolutions, the degree of unsteadiness in the wake increases significantly with increasing yaw angle.

\subsubsection{Wake Interference Effects}

The assessment of wake interference effects on downstream wind turbines in wind farms is crucial for developing accurate predictions of the annual energy yield of a wind farm. In order to demonstrate SMARTROTOR's ability to perform wake interference simulations, a test at $7 \mathrm{~m} / \mathrm{s}$ was performed where identical NREL turbines were separated by 3 rotor diameters as depicted in Figure 7.16(a). As mentioned in Section 4.5, a separation distance of 3 rotor diameters is representative of the spacing between turbines when a wind farm is operating in cross wind conditions. The test conditions that were used can be seen in Table 7.4. The wake interference simulation was

Table 7.4: Wake interference test conditions.

\begin{tabular}{|c|c|}
\hline Turbine Geometry & NREL UAE Phase VI \\
\hline Number of Turbines & 2 \\
\hline Separation distance & $30 \mathrm{~m}$ \\
\hline Rotor speed & $72 \mathrm{rpm}$ \\
\hline Inflow velocity & $7 \mathrm{~m} / \mathrm{s}$ \\
\hline Kinematic viscosity & $1.41 \times 10^{-5} \mathrm{~m}^{2} / \mathrm{s}$ \\
\hline Density & $1.225 \mathrm{~kg} / \mathrm{m}^{3}$ \\
\hline Time step & $0.01 \mathrm{~s} / \mathrm{step}$ \\
\hline
\end{tabular}

performed for 8 turbine revolutions with the particle wake activated, after which the computational time became prohibitive. A single simulation with two turbines required 30 hours on one dual-core $3.2 \mathrm{GHz}$ computer. The results of the simulation 


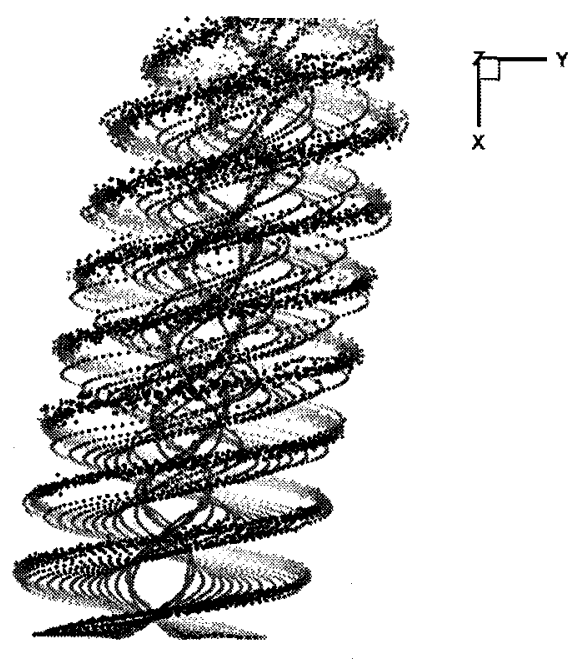

(a) NREL wake at $10^{\circ}$ yaw.

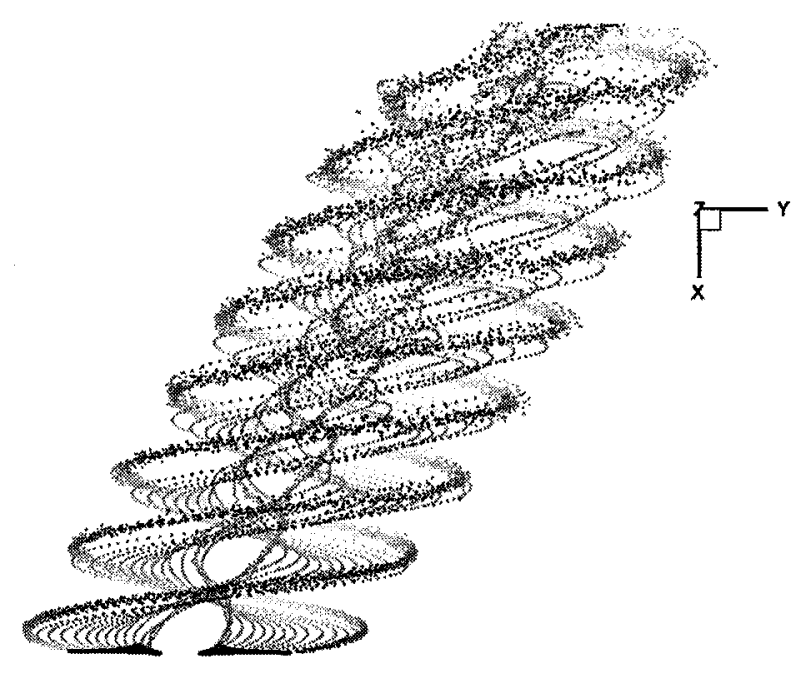

(b) NREL wake at $30^{\circ}$ yaw.

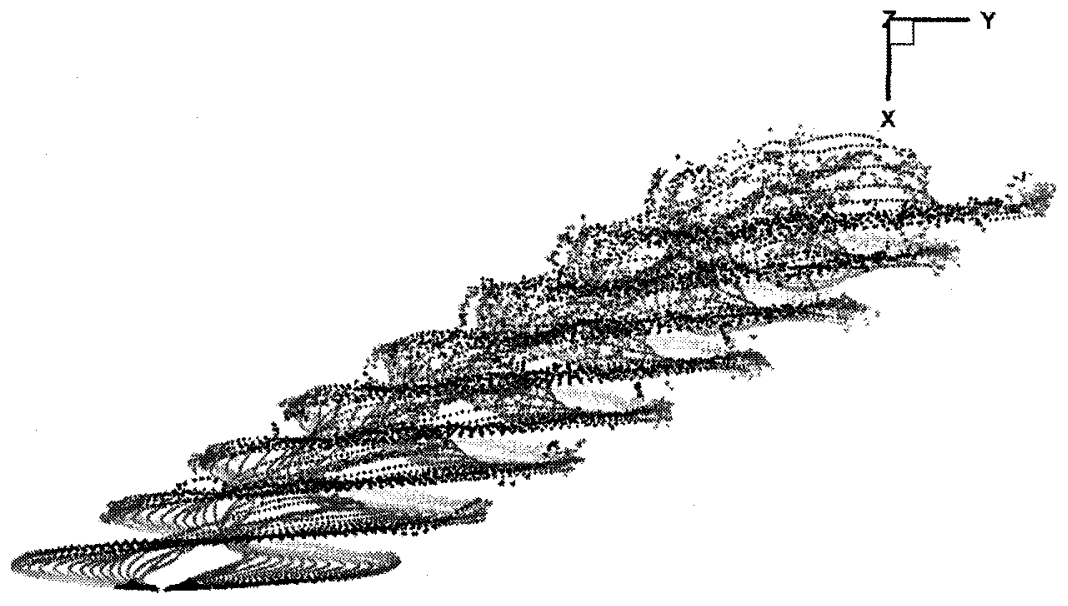

(c) NREL wake at $60^{\circ}$ yaw.

Figure 7.15: Top view of the NREL wake predicted by SMARTROTOR at three yaw angles. 
demonstrated that the power of the downstream turbine is reduced by $50 \%$ in the closely-spaced configuration. The behaviour of the two interacting wakes is shown at the downstream turbine in Figure 7.16(b) where the dark particles represent the wake produced by the upstream turbine and the light particles represent the wake created by the downstream turbine. Experimental results were not available for this test, but it demonstrates SMARTROTOR's ability to perform studies in wind farm behaviour.

\subsection{Conclusions}

SMARTROTOR, an aeroelastic, aeroacoustic, discrete vortex method code, was used to simulate the benchmark NREL wind turbine experiment. The power, torque, and thrust produced by the turbine in normal flow conditions and in yawed flow conditions was evaluated at characteristic operational wind speeds. The predicted wind turbine results in normal flow conditions compared very favourably with the NREL experiment in comparison to other published results. SMARTROTOR has also demonstrated an adequate capability of predicting the power and thrust behaviour of a turbine in yawed flow but is dependent on the accuracy and calibration of the ONERA dynamic stall model.

The use of a grid-free discrete vortex method allows the wake behind a turbine to be resolved rapidly without the need for a very fine CFD mesh and the speed of single turbine simulations ( 3 hours in normal flow conditions on $1 \mathrm{CPU}$ ) is suitable for industrial applications. The versatility and speed of the aeroelastic discrete vortex method was also demonstrated in yawed flow and in wake interaction studies. 


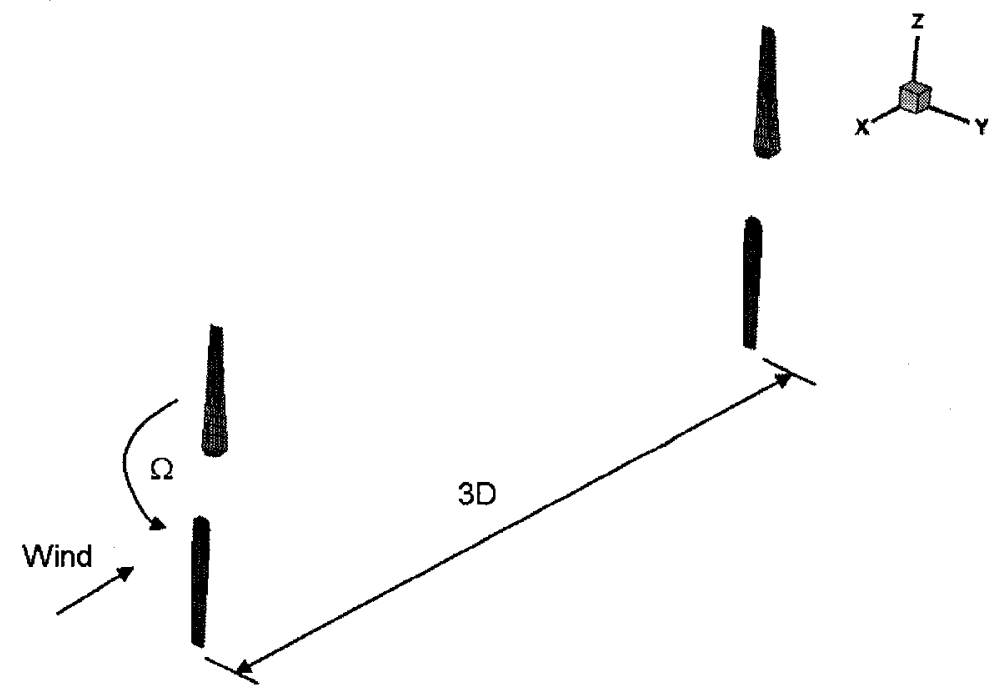

(a) Schematic of the turbine layout, isometric view.

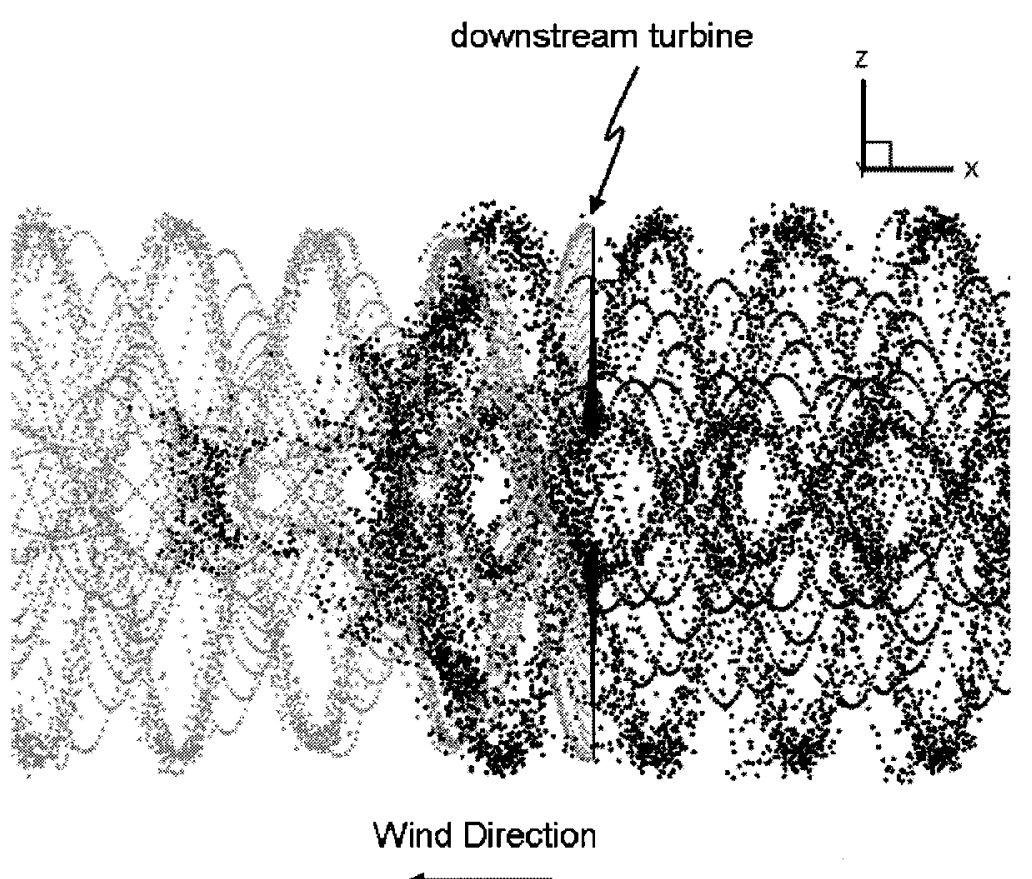

(b) Wake interaction at the downstream turbine, side view.

Figure 7.16: Wake interaction in a closely spaced wind farm configuration. 


\subsection{Recommendations and Future Work}

As the power output predictions of the NREL turbine in yawed flow were overpredicted, additional calibration of the ONERA dynamic stall model should be conducted. The behaviour of the numerical scheme that has been implemented for the ONERA model should be investigated to eliminate the unsteady oscillations in torque and thrust prediction once per cycle.

Additional validation of the wake behaviour and wake ageing predicted by SMARTROTOR should be performed by comparing the wake vortex pitch and radial tip vortex core position with the wind tunnel experiment performed by Haans et al [80] on a scaled NACA 0012 rotor.

Several other non-ideal effects should be included in future simulations to produce more accurate results. This includes the effects of modeling the tower, rotor hub and nacelle, as well as the effect of unsteady inflow velocities and non-uniform inflow to properly represent unsteady loads that will occur due to the atmospheric boundary layer. In the NREL wind turbine simulation, the rotor rpm was prescribed, but the ability of SMARTROTOR to capture turbine startup and a manner by which the rpm can be matched in operational conditions should be evaluated.

The particle mesh technique described by Opoku [3] should be implemented in SMARTROTOR to reduce the number of computations that must be performed at each step from $N^{2}$ to $N \log N$, as this would substantially increase the performance of the code and would increase the speed of multiple turbine simulations.

In 2006, the Energy research Centre of the Netherlands performed a wind tunnel test of a $4.5 \mathrm{~m}$ diameter three-bladed pitch-controlled wind turbine as part of the European Union Measurements and EXperiments In COntrolled conditions (MEXICO) project. As part of the test, particle image velocimetry was used to obtain quantitative information about the velocity field and the tip vortex behaviour. Measurements 
were performed in axisymmetric flow and yawed flow conditions. The results of the study have not been released yet due to ongoing data analysis, but this experiment would allow SMARTROTOR to be validated using pitch-controlled turbine blades.

SMARTROTOR should also be validated on a more realistic commercial turbine with a rotor diameter of 60-100 $\mathrm{m}$. A larger wind turbine rotor would also demonstrate the benefit of more accurate nonlinear structural model (compared to linear beam solvers). This particular test would require rotor blade chord, twist, thickness and airfoil geometry in addition to the structural properties of the blade. The operational conditions of the turbine should also be known, including the expected wind speeds, rotational speed, and blade pitch schedule on a variable-pitch turbine. An extension of this particular test could be to predict the average annual energy production of a wind turbine if the annual wind distribution at a turbine site was known as well. This type of validation would necessitate the cooperation of an organization such as the Wind Energy Institute of Canada (WEICan) as a large amount of operational turbine data would be required.

In order to properly validate SMARTROTOR's ability to predict the velocity deficit in the wake and the power generated by a downstream turbine, an experimental test case should be identified and reproduced. Additionally, experiments with turbines placed side by side or staggered downstream should be investigated. The review of wind turbine experiments performed by Vermeer et al [42] did not identify any experiments involving multiple turbines in a wind farm configuration, but an experiment performed by Maeda et al in 2004 [124] studied the interaction between two HAWTs and should be investigated in greater detail. This could be a precursor to a move to study wind farm effects and wind farm predictions using multiple turbines.

While potential flow discrete vortex methods (DVM) can accurately predict the power output of wind turbines at low to medium speeds, such methods are reliant on two-dimensional airfoil data, dynamic stall models, and stall-delay models, and do 
not properly capture the three-dimensional nature of the stalled flow over the turbine blade. The development of the previously-discussed Hybrid CFD-DVM technique to account for viscous effects over the span of the blade while resolving the wake with vortex particles would be a very large asset and could solve the shortcomings of both CFD and DVM for wind turbine applications. 


\section{References}

[1] R. Beaubien, "Improved frequency domain flutter analysis using computational fluid dynamics," Master's thesis, Carleton University, 2006.

[2] R. Beaubien, F. Nitzsche, and D. Feszty, "Time and frequency domain flutter solutions for the AGARD 445.6 wing.," Proc. of the International Forum on Aeroelasticity and Structural Dynamics, Munich, Germany, 2005.

[3] D. Opoku, "Aeroelastic and aeroacoustic modelling of rotorcraft," Master's thesis, Carleton University, 2002.

[4] D. Hodges and A. Pierce, Introduction to Structural Dynamics and Aeroelasticity. New York, NY: Cambridge University Press, 2006.

[5] I. Garrick and W. H. I. Reed, "Historical development of aircraft flutter," Journal of Aircraft, vol. 18, no. 11, 1981.

[6] "Grob spn back in the air after crash." http://www.flightglobal.com/ articles/2007/03/06/212458/grob-spn-back-in-the-air-after-crash . html, Accessed March 62007.

[7] R. N. Yurkovich, "Status of unsteady aerodynamic prediction for flutter of highperformance aircraft," Journal of Aircraft, vol. 40, no. 5, pp. 832-842, 2003.

[8] C. Borland and D. Rizzetta, "Nonlinear transonic flutter analysis," AIAA Journal, vol. 20, no. 11, 1981.

[9] H. Cunningham, J. Batina, and R. Bennet, "Modern wing flutter analysis by computational fluid dynamics methods," Journal of Aircraft, vol. 25, no. 10, 1988.

[10] R. Lee-Rausch, J. Batina, and H. Yang, "Three-dimensional time-marching aeroelastic analyses using an unstructured-grid Euler method," AIAA Journal, vol. 31, no. 9, pp. 1626-1633, 1993. 
[11] E. Lee-Rausch and J. Batina, "Wing flutter boundary prediction using unsteady Euler aerodynamic method," Journal of Aircraft, vol. 32, no. 2, 1995.

[12] G. S. L. Goura, Time Marching Analysis of Flutter Using Computational Fluid Dynamics. PhD thesis, University of Glasgow, 2001.

[13] C. Farhat, P. Geuzaine, and G. Brown, "Application of a three-field nonlinear fluid-structure formulation to the prediction of the aeroelastic parameters of an F-16 fighter," Computers and Fluids, vol. 32, pp. 3-29, 2003.

[14] K. J. Badcock, B. E. Richards, and M. A. Woodgate, "Elements of computational fluid dynamics on block structured grids using implicit solvers," Progress in Aerospace Sciences, vol. 36, pp. 351-392, 2000.

[15] G. Goura, K. Badcock, M. Woodgate, and B. Richards, "Implicit methods for the time marching analysis of flutter.," The Aeronautical Journal, vol. 105, pp. 169-215, 2001.

[16] "Modeling of turbulence in eros-uk," tech. rep., University of Glasgow, Scotland, 2002.

[17] M. Woodgate, K. Badcock, A. Rampurawala, and B. Richards, "Aeroelastic calculations for the hawk aircraft using the Euler equations," tech. rep., University of Glasgow, Scotland, 2003.

[18] E. Albano and W. Rodden, "A doublet-lattice method for calculating lift distributions on oscillating surfaces in subsonic flows," AIAA Journal, vol. 7, no. 2, pp. 279-285, 1969.

[19] R. G. A. da Silva, A study on correction methods for aeroelastic analysis in transonic flow. PhD thesis, São José dos Campos, 2004.

[20] R. Bisplinghoff, H. Ashley, and R. Halfman, Aeroelasticity. Mineola, NY: Dover Publications, Inc., 1983.

[21] J. Giesing, T. Kalman, and W. Rodden, "Subsonic unsteady aerodynamics for general configurations, direct application of the non-planar doublet-lattice method," tech. rep., Wright-Patterson Air Force Base, 1972.

[22] T. Kalman, W. Rodden, and J. Giesing, "Aerodynamic influence coefficients by the doublet lattice method for interfering non planar lifting surfaces oscillating in a subsonic flow," tech. rep., 1969. 
[23] J. D. J. Anderson, Fundamentals of Aerodynamics. New York: McGraw Hill, 3 ed., 2001.

[24] O. Mello, An improved hybrid Navier-Stokes/Full-Potential Method for Computation of Unsteady Compressible Flows. PhD thesis, Georgia Institute of Technology, 1994.

[25] E. Yates, N. Land, and J. Foughner, "Measured and calculated subsonic and transonic flutter characteristics of a $45^{\circ}$ sweptback wing planform in air and in freon-12 in the Langley transonic dynamics tunnel.," tech. rep., Langley Research Center, Virginia, 1963.

[26] H. Tijdeman and J. W. G. van Nunen, "Transonic wind-tunnel tests on an oscillating wing with external store, part 1: General description," NLR TR 78105 U Part 1, 1978.

[27] R. M. Kolonay, Unsteady Aeroelastic Optimization in the Transonic Regime. $\mathrm{PhD}$ thesis, Purdue University, 1996.

[28] W. P. Rodden and J. D. Revell, "The status of unsteady aerodynamic influence coefficients," Fairchild Fund Paper FF-33, 1962.

[29] K. Gupta, "Development of a finite element aeroelastic analysis capability.," Journal of Aircraft, vol. 33, no. 5, 1996.

[30] C. Liu, Y. Zhu, and et al, "Calculation of wing flutter by a coupled fluidstructure method," Journal of Aircraft, vol. 38, no. 2, 2001.

[31] C. Farhat and M. Lesoinne, "High order staggered and subiteration-free algorithm for nonlinear transient aeroelastic problems," AIAA Journal, vol. 36, no. 9, pp. 1754-1757, 1998.

[32] K. J. Badcock, M. A. Woodgate, and B. Richards, "Direct aeroelastic bifurcation analysis of a symmetric wing based on the Euler equations.," tech. rep., University of Glasgow, Scotland, 2003.

[33] "World wind energy - press release." World Wind Energy Association, February 2008.

[34] E. Hau, Wind Turbines: Fundamentals, Technologies, Application and Economics. Springer, 2 ed., 2005. 
[35] D. A. Spera, ed., Wind Turbine Technology: Fundamental Concepts of Wind Turbine Engineering. New York: ASME Press, 1994.

[36] S. Gupta, Development of a time-accurate viscous Lagrangian vortex wake model for wind turbine applications. PhD thesis, University of Maryland, 2006.

[37] M. S. Chappel, "Wind energy research and development at the national research council canada, 1975-1985," tech. rep., National Research Council Canada, Ottawa, Ontario, Canada, 1986.

[38] S. Guha, H. Soloumah, and N. Kar, "Status of and prospect for wind power generation in canada," Wind Engineering, vol. 29, no. 3, pp. 253-270, 2005.

[39] "Wind farms." http://www.canwea.ca/farms/index_e.php, Accessed May 25 2008.

[40] "Basic principles of wind resource evaluation." http://www.awea.org/faq/ basicwr.html, Accessed May 152008.

[41] J. G. Leishman, "Challenges in modeling the unsteady aerodynamics of wind turbines," Wind Energy, vol. 5, 2002.

[42] J. Vermeer, L., J. Sorensen, and A. Crespo, "Wind turbine wake aerodynamics," Progress in Aerospace Sciences, vol. 39, 2003.

[43] S. Schreck, "The NREL full-scale wind tunnel experiment - introduction to the special issue," Wind Energy, vol. 5, 2002.

[44] S. Gupta and J. Leishman, "Performance predictions of the NREL phase VI combined experiment rotor using a free-vortex wake model," Proc. of the 44th AIAA Aerospace Sciences Meeting and Exhibit, Paper AIAA 2006-390, Reno, Nevada, 2006.

[45] J. G. Leishman, Principles of Helicopter Aerodynamics. Cambridge Aerospace Series, New York: Cambridge University Press, 2 ed., 2006.

[46] "GH WindFarmer validation report," tech. rep., Bristol, England, 2003.

[47] H. M., J. Sorensen, S. Voutsinas, and et al., "State of the art in wind turbine aerodynamics and aeroelasticity," Progress in Aerospace Sciences, vol. 42, 2006. 
[48] D. Simms, S. Schreck, M. Hand, and L. Fingersh, "NREL Unsteady Aerodynamics Experiment in the NASA-Ames wind tunnel: A comparison of predictions to measurements," NREL/TP-500-29494, National Renewable Energy Laboratory, Golden, Colorado, USA, 2001.

[49] I. Katic, J. Hostrup, and N. Jensen, "A simple model for cluster efficiency," Proc. of the European Wind Energy Conference, 1986.

[50] "GH WindFarmer theory manual," tech. rep., Bristol, England, 2007.

[51] J. F. Ainslie, "Calculating the flowfield in the wake of wind turbines," Journal of Wind Engineering and Industrial Aerodynamics, vol. 27, 1988.

[52] B. Lange and et al, "Modeling of offshore wind turbine wakes with the wind farm program FLaP," Proc. of the OWEMES conference, 2003.

[53] J. Ainslie, U. Hassan, and et al, "A wind tunnel investigation of the wake structure within small wind turbine farms," Proc. of the European Wind Energy Conference, P. 547-551, Glasgow, Scotland, 1989.

[54] "Clean energy project analysis: Retscreen engineering and cases." http://www . retscreen.net/ang/12.php, Updated Nov 2 2007. Accessed May 22008.

[55] "Wasp wake model." http://www.wasp.dk/Products/WAsP/ WakeEffectModel.html, Updated Aug 2007. Accessed May 72008.

[56] "WAsP 9 help facility and on-line documentation." http://www.wasp.dk/ Support/FAQ/WebHelp/Wasp9.htm, Updated 2007. Accessed May 72008.

[57] A. Bowen and N. Mortensen, "Exploring the limits of WAsP the wind atlas analysis and application program," Proc. of the European Union Wind Energy Conference, Sweden, May 1996.

[58] "Oldenburg university wind farm layout program FLaP." http://www. forwind.de/gb/produkte_dienstleistungen/flap/index.shtml, Accessed May 72008.

[59] G. Schepers, "Modelling of wake aerodynamics + new farm control strategies," Dutch Wind Workshops, Presentation Slides, October 2006.

[60] W. Schlez and et al., "ENDOW: Improvement of wake models within offshore wind farms," Wind Engineering, vol. 25, no. 5, 2001. 
[61] H. Glauert, "Airplane propellers," in Aerodynamic Theory (W. F. Durand, ed.), vol. 4, (Berlin, Germany), pp. 169-360, Springer-Verlag, 1935.

[62] S. Gupta and J. Leishman, "Comparison of momentum and vortex methods for the aerodynamic analysis of wind turbines,"

[63] M. Hansen, Aerodynamics of Wind Turbines. New York: Earthscan Publications Ltd., 1 ed., 2001.

[64] T. Burton, D. Sharpe, N. Jenkins, and E. Bossanyi, Wind Energy Handbook. New York: John Wiley and Sons, 2001.

[65] D. Molenaar and S. Dijkstra, "State-of-the-art of wind turbine design codes: Main features overview for cost-effective generation," Wind Engineering, vol. 23, 1999.

[66] C. Masson, I. Ammara, and I. Paraschivoiu, "An aerodynamic method for the analysis of isolated horizontal-axis wind turbines," International Journal of Rotating Machines, vol. 3, pp. 21-32, 1997.

[67] T. Wu, "Flow through a heavily loaded actuator disk," Schiffstechnik, vol. 9, pp. 134-138, 1962.

[68] C. Masson, I. Ammara, C. Leclerc, and I. Paraschivoiu, "Numerical simulations of the three-dimensional turbulent flow in a wind park," AIAA Journal - AIAA 98-0058, 1998.

[69] I. Ammara, C. Leclerc, and C. Masson, "A viscous three-dimensional differential/actuator-disk method for aerodynamic analysis of wind farms," Journal of Solar Energy - T ASME, vol. 124, no. 4, pp. 345-356, 2002.

[70] N. Sorensen, J. and C. Kock, "A model for unsteady rotor aerodynamics," Journal of Wind Engineering and Industrial Aerodynamics, vol. 39, pp. 139$149,1992$.

[71] J. Robison, D., F. Coton, R. Galbraith, and M. Vezza, "Application of a prescribed wake aerodynamic prediction scheme to horizontal axis wind turbines in axial flow," Wind Engineering, vol. 19, no. 1, pp. 41-51, 1995.

[72] S. Lain, J. Garcia, and R. Aliod, "Development of a lifting surface - 2D panel method to compute the three-dimensional pressure distribution over the blade of a horizonta-axis wind turbine," Wind Engineering, vol. 19, 1995. 
[73] T. Wang and F. Coton, "An unsteady aerodynamic model for HAWT performance including tower shadow effects," Wind Engineering, vol. 23, 1999.

[74] S. Schmitz and J.-J. Chattot, "Method for aerodynamic analysis of wind turbines at peak power," AIAA Journal of Propulsion and Power-Technical Notes, vol. 23 , no. $1,2007$.

[75] R. Bareiss and S. Wagner, "The free wake/hybrid wake code ROVLM - a tool for the aerodynamic analysis of wind turbines," Proc. of the European Community Wind Energy Conference, P. 424-427, Germany, 1993.

[76] J. Whale, C. G. Anderson, R. Bareiss, and S. Wagner, "An experimental and numerical study of the vortex structure in the wake of a wind turbine," Journal of Wind Engineering and INdustrial Aerodynamics, vol. 84, 2000.

[77] E. Duque, M. Burklund, and W. Johnson, "Navier-Stokes and comprehensive analysis performance predictions of the NREL Phase VI experiment," Proc. of the 41st AIAA Aerospace Sciences Meeting and Exhibit, Paper AIAA 2003-355, Reno, Nevada, 2003.

[78] S. Gupta and J. Leishman, "Validation of a free-vortex wake model for wind turbines in yawed flow," Proc. of the 44th AIAA Aerospace Sciences Meeting and Exhibit, Paper AIAA 2006-389, Reno, Nevada, 2006.

[79] S. Gupta and J. Leishman, "Dynamic stall modeling of the S809 airfoil and comparison with experiments," Proc. of the 44th AIAA Aerospace Sciences Meeting and Exhibit, Paper AIAA 2006-196, Reno, Nevada, 2006.

[80] W. Haans, T. Sant, G. Kuik, and G. Bussel, "Measurement and modelling of tip vortex paths in the wake of a HAWT under yawed flow conditions," Proc. of the 43rd AIAA Aerospace Sciences Meeting and Exhibit, Paper AIAA 2005-590, Reno, Nevada, 2005.

[81] A. Afjeh and T. Keith, "A simplified free wake method for horizontal-axis wind turbine performance prediction," Transactions of the ASME Journal of Fluids Engineering, vol. 108, pp. 303-309, 1986.

[82] F. Simoes and J. Graham, "Preidiction of loading on a horizontal axis wind turbine using a free vortex wake model," Proc. of the GWEA Conference, 1991.

[83] S. Voutsinas, M. Belessis, and S. Huberson, "Dynamic inflow effects and vortex particle methods," Proc. of the European Community Wind Energy Conference, P. 428-431, Germany, 1993. 
[84] D. Opoku, D. Triantos, F. Nitzsche, and S. Voutsinas, "Rotorcraft aerodynamic and aeroacoustic modelling using vortex particle methods," Proc. of the ICAS 2002 Congress, The International Council of the Aeronautical Sciences, Toronto, Canada, 2002.

[85] D. G. Opoku and F. Nitzsche, "Acoustic validation of a new code using particle wake aerodynamics and geometrically-exact beam structural dynamics," Proc. of the 29th European Rotorcraft Forum, Germany, 2003.

[86] C. Cesnik, D. Opoku, F. Nitzsche, and T. Cheng, "Active twist rotor blade modelling using particle-wake aerodynamics and geometrically exact beam structural dynamics," Journal of Fluids and Structures, vol. 19, pp. 651-668, 2004.

[87] N. N. Sorensen, J. Michelsen, and S. Schreck, "Navier-Stokes predictions of the NREL Phase VI rotor in the NASA Ames $80 \mathrm{ft}$ x $120 \mathrm{ft}$ wind tunnel," Wind Energy, vol. 5, 2002.

[88] N. Sezer-Uzol and L. Long, "3-D time-accurate CFD simulations of wind turbine rotor flow fields," Proc. of the 44th AIAA Aerospace Sciences Meeting and Exhibit, Paper AIAA 2006-394, Reno, Nevada, 2003.

[89] F. Massouh, T. Dobrev, and M. Rapin, "Experimental and numerical survey in the wake of a wind turbine," Proc. of the 45th AIAA Aerospace Sciences Meeting and Exhibit, Paper AIAA 2007-423, Reno, Nevada, 2007.

[90] S. Benjanirat and L. Sankar, "Evaluation of turbulence models for the prediction of wind turbine aerodynamics," Proc. of the 41st AIAA Aerospace Sciences Meeting and Exhibit, Paper AIAA 2003-0517, Reno, Nevada, 2003.

[91] C. Tongchitpakdee, S. Benjanirat, and L. Sankar, "Nmerical simulation of the aerodynamics of horizontal axis wind turbines under yawed flow conditions," Proc. of the 43rd AIAA Aerospace Sciences Meeting and Exhibit, Paper AIAA 2005-773, Reno, Nevada, 2005.

[92] G. Xu, Computational Studies of Horizontal Wind Turibnes. PhD thesis, Georgia Institute of Technology, 2001.

[93] S. Benjanirat and L. Sankar, "Recent improvements to a combined navier-stokes full potential methodology for modeling horizontal axis wind turbines," 42nd AIAA Aerospace Sciences Meeting and Exhibit, Paper AIAA 2004-830, Reno, Nevada, 2004. 
[94] P. Chaviaropoulos, "Development of a state-of-the-art aeroelastic simulator for horizontal axis wind turbines part 1: Structural aspects," Wind Engineering, vol. 20, no. 6, pp. 405-421, 1996.

[95] A. Hansen and D. Laino, "Validation study for AeroDyn and YawDyn using phase III combined experiment data (prediction of turbine dynamics)," Proc. of the 35th AIAA Aerospace Sciences Meeting and Exhibit, Paper 97-0943, Reno, Nevada, 1997.

[96] D. Hodges, "Review of composite rotor blade modeling," AIAA Journal - Technical Notes, vol. 28, no. 3, pp. 561-565, 1999.

[97] M. Hand, D. Simms, L. Fingersh, and et al., "Unsteady Aerodynamics Experiment Phase VI: Wind tunnel test configuration and available data campaigns," NREL/TP-500-29955, National Renewable Energy Laboratory, Golden, Colorado, USA, 2001.

[98] S. Voutsinas, M. Belessis, and K. Rados, "Investigation of the yawed operation of wind turbines by means of a vortex particle method," tech. rep.

[99] S. Voutsinas and D. Triantos, "High-resolution aerodynamic analysis of full helicopter configurations," Proc. of the 25th European Rotorcraft Forum, Rome, Italy, 1999.

[100] S. Voutsinas, "A generalized unsteady vortex particle method for solving the unsteady flow around multi-component configurations," tech. rep., National Technical University of Athens, Athens, Greece, 1990.

[101] J. Marshall, Inviscid Incompressible Flow. Wiley, 2001.

[102] J. Hess and A. Smith, "Calculation of potential flow about arbitrary bodies," Progress in Aeronautical Sciences, vol. 8.

[103] C. Rehbach, "Calcul d'ecoulements autour d'ailes sans epaisseur avec nappes tourbillonnaires evolutives," la Recherche Aerospatiale, no. 2, pp. 53-61, 1973.

[104] J. Beale and A. Majda, "Vortex methods I: Convegence in three dimensions," Mathematics of Computation, vol. 39, no. 159, pp. 1-27, 1982.

[105] J. Beale and A. Majda, "High order accurate vortex methods with explicit velocity kernels," Journal of Computational Physics, vol. 58, pp. 188-208, 1985. 
[106] J. Larsen, S. Nielsen, and S. Krenk, "Dynamic stall model for wind turbine airfoils," Journal of Fluids and Structures, vol. 23, pp. 959-982, 2007.

[107] C. T. Tran and D. Petot, "Semi-empirical model for the dynamic stall of airfoils in view to the application to the calculation of responses of a helicopter blade in forward flight," Vertica, vol. 5, no. 1, pp. 35-53, 1981.

[108] D. Gross and F. Harris, "Prediction of in-flight stalled airloads from oscillating airfoil data," Proc. of the 25th annual forum of the American Helicopter Society, Washington DC, 1969.

[109] J. Leishman and T. Beddoes, "A semi-empirical model for dynamic stall," Journal of the American Helicopter Society, vol. 34, no. 3, pp. 3-17, 1989.

[110] S. Voutsinas, V. Riziotis, and P. Chaviaropoulos, "Non-linear aerodynamics and fatigue loading on wind turbines operating at extreme sites," Proc. of the 35th AIAA Aerospace Sciences Meeting and Exhibit, Paper AIAA 97-0935, Reno, Nevada, 1997.

[111] D. Petot, "Differential equation modeling of dynamic stall," La Recherche Aerospatiale, vol. 5, pp. 59-72, 1989.

[112] D. Peters, "Toward a unified lift model for use in rotor blade stability analysis," Journal of the American Helicopter Society, vol. 30, pp. 32-42, 1985.

[113] W. Bierbooms, "A comparison between unsteady aerodynamic models," Journal of Wind Engineering and Industrial Aerodynamics, vol. 39, pp. 23-33, 1992.

[114] D. Tang and E. Dowell, "Comments on the ONERA stall aerodynamic model and its impact on aeroelastic stability," Journal of Fluids and Structures, vol. 10, pp. 353-366, 1996.

[115] K. McAlister, O. Lambert, and D. Petot, "Application of the ONERA model of dynamic stall," NASA technical paper 2399, National Aeronautics and Space Administration, 1984.

[116] D. Hodges, "A mixed variational formulation based on exact intrinsic equations for dynamics of moving beams," International Journal of Solids and Structures, vol. 26 , no. 11 , pp. 1253-1273, 1990.

[117] X. Shang, Aeroelastic Stability of Composite Hingeless Rotors with Finite-State Unsteady Aerodynamics. PhD thesis, Georgia Institute of Technology, 1995. 
[118] T. Cheng, "Structural dynamics modelling of helicopter blades for computational aeroelasticity," Master's thesis, Massachusetts Institute of Technology, 2002.

[119] S. Shin, Integral Twist Actuation of Helicopter Rotor Blades for Vibration Reduction. PhD thesis, Massachusetts Institute of Technology, 2001.

[120] W. H. Press, Numerical Recipes in FORTRAN 77: The Art of Scientific Computing. New York: Cambridge University Press, 1992.

[121] D. Somers, "Design and experimental results for the s809 airfoil," NREL/SR440-6918, National Renewable Energy Laboratory, Golden, Colorado, USA, 1997.

[122] R. Ramsay, M. Hoffman, and G. Gregorek, "Effects of grit roughness and pitch oscillations on the 8809 airfoil," NREL/TP-442-7817, National Renewable Energy Laboratory, Golden, Colorado, USA, 1995.

[123] V. Riziotis, S. Voutsinas, and A. Zervos, "Investigation of the yaw induced stall and its impact to the design of wind turbines," tech. rep., National Technical University of Athens, Athens, Greece.

[124] T. Maeda, T. Yokota, Y. Shimizu, and K. Adachi, "Wind tunnel study of the interaction between two horizontal axis wind turbines," Wind Engineering, vol. 28, no. 2, pp. 197-209, 2004. 


\section{Appendix A}

\section{Derivation of the AIC-correction Fourier}

\section{Transform}

In order to transform the nonlinear lifting pressures obtained from rigid body oscillatory pitch CFD simulations into the frequency domain, the unsteady pressures must be Fourier transformed, following which they can be used to correct the linear frequency domain solution to account for nonlinear transonic effects. The following derivation has been reproduced from the work of Mello [24] for completeness and was used by Silva [19] in a similar AIC-correction procedure.

The unsteady oscillatory pitch simulations were performed about a mean angle of $0^{\circ}$. The complex representation of the motion of the wing during the CFD simulations is given by Equation A.1

$$
\alpha^{C}(\tau)=\Delta \alpha e^{i k \tau}
$$

where $\tau$ is the non-dimensional time step, $k$ is the reduced frequency of the pitching motion, $\alpha$ is the angle of attack, and the superscript ${ }^{C}$ is the complex representation of the angle of attack at a particular time step. The complex representation of the steady-state response is given by Equation A.2.

$$
C_{P}^{C}(\tau)=\left[\operatorname{Re}\left(C_{P i}\right)+i \operatorname{Im}\left(C_{P i}\right)\right] e^{i k \tau}
$$


The actual motion of the wing is described by the imaginary part of Equation A.1 and so the actual response is described by the imaginary part of Equation A.2. The actual response $C_{P}(\tau)$ can be related to the complex representation by Equation A.3

$$
\begin{aligned}
& C_{P}^{C}(\tau)=\left[\operatorname{Re}\left(C_{P i}\right)+i \operatorname{Im}\left(C_{P i}\right)\right] e^{i k \tau} \\
& =\left[\operatorname{Re}\left(C_{P i}\right) \cos (k \tau)-\operatorname{Im}\left(C_{P i}\right) \sin (k \tau)\right]+i\left[\operatorname{Re}\left(C_{P i}\right) \sin (k \tau)+\operatorname{Im}\left(C_{P i}\right) \cos (k \tau)\right]
\end{aligned}
$$

The imaginary portion of Equation A.3 corresponds to the pressure coefficient response, as described by Equation A.4.

$$
C_{P}(\tau)=\left[\operatorname{Re}\left(C_{P i}\right) \sin (k \tau)+\operatorname{Im}\left(C_{P i}\right) \cos (k \tau)\right]
$$

The real and imaginary components of the unsteady pressure at a particular spanwise location $i$ can be obtained in terms of the actual pressure coefficient response described by Equations A.5 and A.6.

$$
\begin{aligned}
& \operatorname{Re}\left(C_{P i}\right)=\frac{k}{\pi} \int_{\tau_{1}}^{\tau_{1}+2 \pi / k} C_{P}(\tau) \sin (k \tau) d \tau \\
& \operatorname{Im}\left(C_{P i}\right)=\frac{k}{\pi} \int_{\tau_{1}}^{\tau_{1}+2 \pi / k} C_{P}(\tau) \cos (k \tau) d \tau
\end{aligned}
$$

In Equations A.5 and A.6, $\tau_{1}$ should be selected so that the transient portion of the unsteady response is not included in the Fourier transform. The real and imaginary components of the lifting pressures are determined from the discrete Fourier transform 
of the above two Equations, as seen in Equations A.7 and A.8

$$
\begin{aligned}
& \operatorname{Re}\left(C_{P i}\right)=\frac{k \Delta \tau}{2 \pi \Delta \alpha} \sum_{m=m_{1}}^{m_{1}+m_{T}} C_{P m} \sin (k m \Delta \tau) \\
& \operatorname{Im}\left(C_{P i}\right)=\frac{k \Delta \tau}{2 \pi \Delta \alpha} \sum_{m=m_{1}}^{m_{1}+m_{T}} C_{P m} \cos (k m \Delta \tau)
\end{aligned}
$$

where $m$ refers to the time step number, $m_{1}=\tau_{1} / \Delta \tau$ and $m_{T}=2 \pi / k \Delta \tau$. The above two Equations have been normalized with respect to the amplitude of oscillation $2 \Delta \alpha$ and it should be noted that the discrete Fourier transform used in this work as well as that of Silva [19] includes the Mach number $(M)$ in the numerator as well. 


\section{Appendix B}

\section{Geometric and Structural Properties of the NREL UAE Phase VI Wind Turbine}

The model of the NREL turbine blade was created based on the geometric and structural information provided by the NREL in [97]. The experimental NREL blade begins at a radius $r=0.508 \mathrm{~m}$ from the centre of the hub with a cylindrical section until $r=0.883 \mathrm{~m}$. The blade then transitions from a cylindrical cross-section to the S809 airfoil at $r=1.257 \mathrm{~m}$. In order to simplify the blade for SMARTROTOR, the cylindrical and transitional sections were neglected and the root of the blade model was initiated at $r=1.008 m$ with the $\mathrm{S} 809$ airfoil.

The chord and twist distributions of the NREL blade were interpolated to the 17 spanwise panel nodes, as seen in Table B.1, but the blade thickness to chord ratio of $21 \%$ was neglected due to the thin wing representation of the airfoil. The twist distribution described in Table B.1 is positive towards the feathered blade position, and the tip pitch angle was set to $3^{\circ}$ for the simulations.

The structural properties of the NREL blade were provided in [97] and were interpolated to the centre of each of the 16 elements of the structural model. The mass and stiffness data required at each radial strip in SMARTROTOR includes the principal mass moments of inertia, the directional mass distribution on each element, 
Table B.1: Interpolated geometric parameters for the NREL turbine blade.

\begin{tabular}{|c|c|c|}
\hline $\begin{array}{c}\text { Radius } \\
{[\mathrm{m}]}\end{array}$ & $\begin{array}{c}\text { Twist } \\
{[\mathrm{deg}]}\end{array}$ & $\begin{array}{c}\text { Chord } \\
{[\mathrm{m}]}\end{array}$ \\
\hline 1.008 & 6.7 & 0.349 \\
\hline 1.067 & 9.9 & 0.441 \\
\hline 1.133 & 13.4 & 0.544 \\
\hline 1.257 & 20.04 & 0.737 \\
\hline 1.648 & 11.909 & 0.697 \\
\hline 1.952 & 7.979 & 0.666 \\
\hline 2.257 & 5.308 & 0.636 \\
\hline 2.562 & 3.425 & 0.605 \\
\hline 2.867 & 2.083 & 0.574 \\
\hline 3.172 & 1.150 & 0.543 \\
\hline 3.598 & 0.385 & 0.500 \\
\hline 4.023 & -0.381 & 0.457 \\
\hline 4.391 & -0.920 & 0.420 \\
\hline 4.696 & -1.352 & 0.389 \\
\hline 4.863 & -1.564 & 0.374 \\
\hline 4.946 & -1.669 & 0.366 \\
\hline 5.029 & -1.775 & 0.358 \\
\hline
\end{tabular}


the axial stiffness, the torsional stiffness, the flapwise and lead-lag stiffness, and the elastic axis of the wing. The structural properties used to model the NREL blade span can be seen Table B.2.

The third component to the NREL blade model was two-dimensional airfoil data for the S809 airfoil that was used for viscous corrections to the potential forces determined with SMARTROTOR. The NREL report [97] included two-dimensional airfoil data obtained from an experiment performed at the Ohio State University [122]. The two-dimensional wind tunnel test performed on the S809 in Reference [122] contained data at Reynolds numbers from 0.75 to 1.5 million. The lift, drag, and moment coefficients were available from $\alpha=-20^{\circ}$ to $\alpha=+26^{\circ}$ at Reynolds numbers of 0.75 $\times 10^{6}, 1.0 \times 10^{6}$, and $1.5 \times 10^{6}$. However, at a Reynolds number of $1.25 \times 10^{6}$, two-dimensional coefficients were available up to $+40^{\circ}$. As the S809 airfoil did not exhibit high sensitivity to the Reynolds number in [122], the coefficients at high angle of attack (above $26^{\circ}$ ) at a Reynolds number of $1.25 \times 10^{6}$ were used to extend the data sets at the other three Reynolds numbers. 
Table B.2: Interpolated structural parameters for the NREL turbine blade.

\begin{tabular}{|c|c|c|c|c|c|c|c|c|c|}
\hline Radius $[m]$ & Panel Midpoint & Mass & $I_{x x}$ & $I_{y y}$ & $I_{z z}$ & $E A$ & $G J$ & $E I_{\text {flap }}$ & $E I_{l a g}$ \\
\hline 1.008 & {$[m]$} & {$[k g]$} & {$\left[\mathrm{kgm}^{4}\right]$} & {$\left[\mathrm{kgm}^{4}\right]$} & {$\left[\mathrm{kgm}^{\mathbf{4}}\right]$} & {$[N]$} & {$\left[N m^{2}\right]$} & {$\left[N m^{2}\right]$} & {$\left[N m^{2}\right]$} \\
\hline 1.067 & 1.038 & 0.742 & $3.906 \mathrm{E}-02$ & $2.250 \mathrm{E}-01$ & $2.640 \mathrm{E}-01$ & $4.00 \mathrm{E}+08$ & $1.55 \mathrm{E}+6$ & $1.52 \mathrm{E}+6$ & $3.95 \mathrm{E}+6$ \\
\hline 1.133 & 1.100 & 0.899 & $3.876 \mathrm{E}-02$ & 2.999E-01 & $3.386 \mathrm{E}-01$ & $4.02 \mathrm{E}+08$ & $2.01 \mathrm{E}+6$ & $1.47 \mathrm{E}+6$ & $4.88 \mathrm{E}+6$ \\
\hline 1.257 & 1.195 & 1.888 & $3.830 \mathrm{E}-02$ & 4.137E-01 & $4.520 \mathrm{E}-01$ & $4.05 \mathrm{E}+08$ & $2.71 \mathrm{E}+6$ & $1.40 \mathrm{E}+6$ & $6.29 \mathrm{E}+6$ \\
\hline 1.648 & 1.453 & 6.177 & $3.496 \mathrm{E}-02$ & $4.603 \mathrm{E}-01$ & $4.953 \mathrm{E}-01$ & $3.90 \mathrm{E}+08$ & $2.95 \mathrm{E}+6$ & $1.21 \mathrm{E}+6$ & $7.01 \mathrm{E}+6$ \\
\hline 1.952 & 1.800 & 4.553 & $2.970 \mathrm{E}-02$ & $4.115 \mathrm{E}-01$ & $4.412 \mathrm{E}-01$ & $3.62 \mathrm{E}+08$ & $2.59 \mathrm{E}+6$ & $9.93 \mathrm{E}+5$ & $6.59 \mathrm{E}+6$ \\
\hline 2.257 & 2.105 & 4.441 & $2.605 \mathrm{E}-02$ & $3.765 \mathrm{E}-01$ & $4.026 \mathrm{E}-01$ & $3.54 \mathrm{E}+08$ & $2.32 \mathrm{E}+6$ & $8.60 \mathrm{E}+5$ & $6.36 \mathrm{E}+6$ \\
\hline 2.562 & 2.410 & 3.713 & $1.908 \mathrm{E}-02$ & $2.673 \mathrm{E}-01$ & $2.864 \mathrm{E}-01$ & $3.12 \mathrm{E}+08$ & $1.57 \mathrm{E}+6$ & $6.86 \mathrm{E}+5$ & $4.82 \mathrm{E}+6$ \\
\hline 2.867 & 2.715 & 3.440 & $1.565 \mathrm{E}-02$ & $2.245 \mathrm{E}-01$ & $2.402 \mathrm{E}-01$ & $2.74 \mathrm{E}+08$ & $1.33 \mathrm{E}+6$ & $5.43 \mathrm{E}+5$ & $3.98 \mathrm{E}+6$ \\
\hline 3.172 & 3.020 & 3.223 & $1.305 \mathrm{E}-02$ & $1.870 \mathrm{E}-01$ & $2.001 \mathrm{E}-01$ & $2.46 \mathrm{E}+08$ & $1.12 \mathrm{E}+6$ & $4.43 \mathrm{E}+5$ & $3.29 \mathrm{E}+6$ \\
\hline 3.598 & 3.385 & 4.168 & $1.042 \mathrm{E}-02$ & $1.491 \mathrm{E}-01$ & $1.595 \mathrm{E}-01$ & $2.17 \mathrm{E}+08$ & $9.10 \mathrm{E}+5$ & $3.46 \mathrm{E}+5$ & $2.60 \mathrm{E}+6$ \\
\hline 4.023 & 3.810 & 3.775 & $7.687 \mathrm{E}-03$ & $1.118 \mathrm{E}-01$ & $1.195 \mathrm{E}-01$ & $1.82 \mathrm{E}+08$ & $6.93 \mathrm{E}+5$ & $2.43 \mathrm{E}+5$ & $1.92 \mathrm{E}+6$ \\
\hline 4.391 & 4.207 & 2.961 & $5.649 \mathrm{E}-03$ & $8.380 \mathrm{E}-02$ & $8.945 \mathrm{E}-02$ & $1.51 \mathrm{E}+08$ & $5.27 \mathrm{E}+5$ & $1.69 \mathrm{E}+5$ & $1.42 \mathrm{E}+6$ \\
\hline 4.696 & 4.544 & 2.244 & $4.225 \mathrm{E}-03$ & $6.375 \mathrm{E}-02$ & $6.798 \mathrm{E}-02$ & $1.26 \mathrm{E}+08$ & $4.07 \mathrm{E}+5$ & $1.19 \mathrm{E}+5$ & $1.06 \mathrm{E}+6$ \\
\hline 4.863 & 4.779 & 1.146 & $3.372 \mathrm{E}-03$ & $5.163 \mathrm{E}-02$ & $5.501 \mathrm{E}-02$ & $1.09 \mathrm{E}+08$ & $3.34 \mathrm{E}+5$ & $9.05 \mathrm{E}+4$ & $8.45 \mathrm{E}+5$ \\
\hline 4.946 & 4.904 & 0.552 & $2.981 \mathrm{E}-03$ & $4.596 \mathrm{E}-02$ & $4.894 \mathrm{E}-02$ & $1.00 \mathrm{E}+08$ & $2.99 \mathrm{E}+5$ & $7.78 \mathrm{E}+4$ & $7.46 \mathrm{E}+5$ \\
\hline 5.029 & 4.987 & 0.538 & $2.720 \mathrm{E}-03$ & $4.217 \mathrm{E}-02$ & $4.489 \mathrm{E}-02$ & $9.44 \mathrm{E}+07$ & $2.76 \mathrm{E}+5$ & $6.92 \mathrm{E}+4$ & $6.81 \mathrm{E}+5$ \\
\hline
\end{tabular}




\section{Appendix C}

\section{Implementation of the ONERA dynamic stall model}

The original ONERA dynamic stall model was implemented in GENUVP and modified in SMARTROTOR for the S809 airfoil. The dynamic stall model was developed for pitching motions though it can be used for more general motions. An aerodynamic coefficient $C$ is expressed as the sum of two terms in the ONERA dynamic stall model; one term $C_{1}$ to account for the attached component of the flow and a second term $C_{2}$ to account for the separated flow, as seen in Equation C.1.

$$
C=C_{1}+C_{2}
$$

The lift, drag, and pitching moment models will be presented below with the corresponding discretization, following which the coefficients used in the current work will be shown. The subscripts $z, x$, and $m$ in the following sections refer to the lift, drag, and pitching moment coefficients, respectively, for consistency with the original ONERA report [111]. In the original report however, the angle of attack is written as $\Theta$ whereas $\alpha$ will be used here. 


\section{Lift Model}

The lift coefficient is modeled as the sum of the attached flow component and the separated flow component, as seen in Equation C.2.

$$
C z=C z_{1}+C z_{2}
$$

The differential equations for lift in attached flow and separated flow can be seen in Equation C.3

$$
\begin{aligned}
& \dot{C} z_{1}+\lambda C z_{1}=\lambda C z_{\text {lin }}+(\lambda s+\sigma) \dot{\alpha}+s \ddot{\alpha} \\
& \ddot{C} z_{2}+a \dot{C} z_{2}+r C z_{2}=-[r \Delta C z+E \dot{\alpha}]
\end{aligned}
$$

where $C z_{\text {lin }}$ is the lift created by an idealized linear portion of the lift curve (i.e. only considering the lift curve slope) and $\Delta C z$ is the difference between the idealized linear lift and the lift due to static stall conditions as illustrated in Figure C.1. The lift coefficient for static stall conditions and the linear lift coefficient are both determined based on the input two-dimensional airfoil data. In Equation C.3, the coefficients $\sigma, r, a$, and $E$ can be calculated using Equation C.4. The coefficients for the lift described in Equation C.4 will be presented below.

$$
\begin{aligned}
& \sigma=\sigma_{0}+\sigma_{1}|\Delta C z| \\
& \sqrt{r}=r_{0}+r_{2} \Delta C z^{2} \\
& a=a_{0}+a_{2} \Delta C z^{2} \\
& E=E_{2} \Delta C z^{2}
\end{aligned}
$$

The discretized equations that can be used to calculate the first and second derivatives of the angle of attack $(\alpha)$ seen in Equation C.3 are given below in Equation C.5. 


$$
\begin{gathered}
\dot{\alpha}=\frac{\partial \alpha}{\partial t}=\frac{\alpha_{i}-\alpha_{i-1}}{\Delta t} \\
\ddot{\alpha}=\frac{\partial^{2} \alpha}{\partial t^{2}}=\frac{\alpha_{i}-2 \alpha_{i-1}+\alpha_{i-2}}{\Delta t^{2}}
\end{gathered}
$$

The first and second derivatives of the lift coefficient are discretized using backward difference schemes as seen in Equation C.6 and can be used to resolve the attached and separated flow equations seen above in Equation C.3.

$$
\begin{gathered}
\dot{C} z=\frac{\partial C z}{\partial t}=\frac{C z_{i-2}-4 C z_{i-1}+3 C z_{i}}{2 \Delta t} \\
\ddot{C} z=\frac{\partial^{2} C z}{\partial t^{2}}=\frac{2 C z_{i}-5 C z_{i-1}+4 C z_{i-2}-C z_{i-3}}{\Delta t^{2}}
\end{gathered}
$$

The resulting lift coefficient modeled with the ONERA dynamic stall model can then be calculated with Equation C.2 and returned to the main calling program.
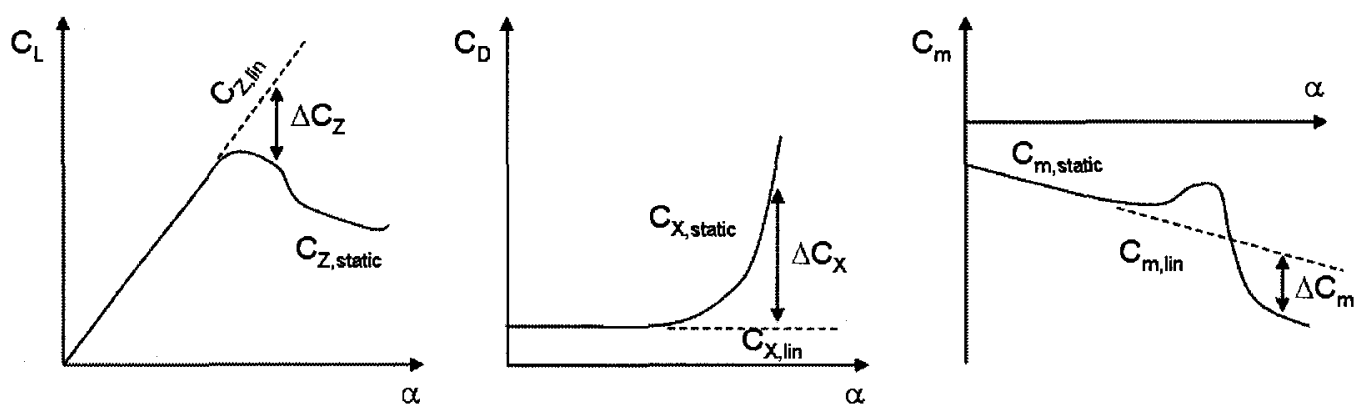

Figure C.1: Lift, Drag, and Moment curves used in the ONERA model.

\section{Drag Model}

The drag coefficient is calculated in the ONERA dynamic stall model in a manner very similar to the lift coefficient. The corrected coefficient is seen in Equation C.7 as the sum of attached and separated flow components.

$$
C x=C x_{1}+C x_{2}
$$


The attached flow and separated flow equations that are used for the drag coefficient are presented in Equation C.8. The attached flow term, unlike the lift term, does not contain a time derivative for the drag coefficient and $C x_{1}$ can be solved directly. The definitions of $C x_{l i n}$ and $\Delta C x$ can be seen in Figure C.1. The coefficients and angle of attack for the drag coefficient are solved using the same equations as for the lift coefficient (see Equations C.4 and C.5). The coefficients themselves however, are slightly different for the drag than for the lift component as seen below. A backward difference method is used to calculate the separated flow term of the drag coefficient as seen in Equation C.9.

$$
\begin{gathered}
C x_{1}=C x_{l i n}+(s+\sigma) \dot{\alpha}+s \ddot{\alpha} \\
\ddot{C} x_{2}+a \dot{C} x_{2}+r C x_{2}=-[r \Delta C x+E \dot{\alpha}] \\
\ddot{C} x=\frac{\partial^{2} C x}{\partial t^{2}}=\frac{2 C x_{i}-5 C x_{i-1}+4 C x_{i-2}-C x_{i-3}}{\Delta t^{2}}
\end{gathered}
$$

\section{Pitch Model}

The pitching moment coefficient is also calculated in the ONERA dynamic stall model in a manner very similar to the lift coefficient. The corrected coefficient is seen in Equation C.10 as the sum of attached and separated flow components.

$$
C m=C m_{1}+C m_{2}
$$

The attached flow and separated flow equations that are used for the pitch coefficient are presented in Equation C.11. The attached flow term, unlike the lift term, does not contain a time derivative term and $C m_{1}$ can be solved directly. The definitions of $C m_{l i n}$ and $\Delta C m$ can be seen in Figure C.1. The coefficients and angle of attack for 
the drag coefficient are solved using the same equations as for the lift coefficient (see Equations C.4 and C.5). The coefficients themselves however, are slightly different for the pitching moment than for the lift and drag components as seen below. A backward difference method is used to calculate the separated flow term of the pitch coefficient as seen in Equation C.12.

$$
\begin{gathered}
C m_{1}=C m_{l i n}+(s+\sigma) \dot{\alpha}+s \ddot{\alpha} \\
\ddot{C} m_{2}+a \dot{C} m_{2}+r C m_{2}=-[r \Delta C m+E \dot{\alpha}] \\
\ddot{C} m=\frac{\partial^{2} C m}{\partial t^{2}}=\frac{2 C m_{i}-5 C m_{i-1}+4 C m_{i-2}-C m_{i-3}}{\Delta t^{2}}
\end{gathered}
$$

\section{ONERA Coefficients}

The ONERA model requires a total of 27 coefficients to describe the time lag behaviour of the lift, drag, and moment coefficients when in dynamic stall conditions. Petot had noted that the coefficients are approximately independent of the selected airfoil, and has suggested coefficients for a mean airfoil if coefficients cannot be determined using wind tunnel experiments [111]. As exact coefficients have only been determined for a few airfoils, it is common practice to use the mean airfoil coefficients determined for the ONERA model. Two of the coefficients that describe the lift behaviour of the model were calibrated in this work for the S809 unsteady pitching experiment performed by the Ohio State University [122]. The 27 coefficients can be seen below in Table C.1. The two coefficients that were changed from the original implementation in GENUVP are indicated in italics, with the original value in parentheses. In the work of Petot, acceptable ranges for the coefficients are provided and the two model coefficients that were calibrated are within the suggested range. 
Table C.1: ONERA dynamic stall coefficients.

\begin{tabular}{|c|c|c|c|}
\hline & Lift & Drag & Pitch \\
\hline$\lambda$ & $0.27(0.2)$ & 0 & 0. \\
\hline$s$ & 0.05 & 0 & $s+\sigma_{0}$ \\
$\sigma_{0}$ & $0.09(0.05)$ & $0.0005 \alpha$ & $-1.1 \pi / 2$ \\
\hline$\sigma_{1}$ & 0.0 & 0 & 0.1 \\
\hline$r_{0}$ & 0.2 & 0.2 & 0.2 \\
\hline$r_{2}$ & 0.2 & 0.2 & 0.2 \\
\hline$a_{o}$ & 0.3 & 0.25 & 0.3 \\
\hline$a_{2}$ & 0.2 & 0.0 & 0.2 \\
\hline$E_{2}$ & -0.05 & -0.02 & 0.03 \\
\hline
\end{tabular}




\section{Appendix D}

\section{Changes made to the SMARTROTOR Source Code}

\section{S809 Airfoil}

SMARTROTOR uses a thin wing representation for lifting surfaces where the blade or wing is modeled by the mean camber line. SMARTROTOR has built-in routines to determine the mean camber line of any NACA 4-digit or 5-digit airfoil. The mean camber line for the S809 airfoil was included in SMARTROTOR as a new airfoil type. The mean camber line was calculated by importing the S809 airfoil surface coordinates from [121]. The mean camber line was calculated as

$$
y_{c}=\frac{S 809_{\text {upper }}+S 809_{\text {lower }}}{2}
$$

As polynomial description of the camber line is required by SMARTROTOR, a fifth order polynomial was fit to the points calculated with Equation D.1 and is described by Equation D.2.

$$
y_{c}=0.5485 x^{5}-1.6841 x^{4}+1.6949 x^{3}-0.6206 x^{2}+0.0613 x+0.0005
$$


A new subroutine called $S 809 W O$ was included in the file geomet-3_sp.f (lines 13441372), where $J$ is the spanwise location. The $\mathrm{S} 809$ airfoil can be accessed by setting the input variable IFTYP found in the .bld input file to a value of 8 . An additional if-statement was added in geomet-3_sp.f at line 405 so that an IFTYP input of 8 will correspond to the $S 809 W O$ subroutine.

\section{Angle of Attack Convention}

The angle of attack convention for wind turbines [34] is typically in the opposite sense of that for helicopter rotors [45]. As such, the sign in the effective angle of attack equation (line 1449 in rescpv-3_sp.coupled2.f) was changed from a negative value (for helicopters or wings) to a positive value (for wind turbines). This should eventually be incorporated as an input to the code. 